\title{
Stability of the optimal filter in continuous time: beyond the Beneš filter
}

\author{
Bui, Van Bien \& Rubenthaler, Sylvain
}

January 27, 2020

Laboratoire J. A. Dieudonné, Université de Nice Sophia Antipolis, Parc Valrose, 06108 Nice cedex 2, FRANCE (bienmaths@gmail.com, rubentha@unice.fr)

\begin{abstract}
We are interested in the optimal filter in a continuous time setting. We want to show that the optimal filter is stable with respect to its initial condition. We reduce the problem to a discrete time setting and apply truncation techniques coming from [OR05]. Due to the continuous time setting, we need a new technique to solve the problem. In the end, we show that the forgetting rate is at least a power of the time $t$. The results can be re-used to prove the stability in time of a numerical approximation of the optimal filter.
\end{abstract}

filtering, signal detection, inference from stochastic processes.

\section{Introduction}

\subsection{Exposition of the problem}

We are given a probability space $(\Omega, \mathcal{F}, \mathbb{P})$. We are interested in the processes $\left(X_{t}\right)_{t \geq 0}$ and $\left(Y_{t}\right)_{t \geq 0}$, solutions of the following SDE's in $\mathbb{R}$

$$
\begin{gathered}
X_{t}=X_{0}+\int_{0}^{t} f\left(X_{s}\right) d s+V_{t}, \\
Y_{t}=\int_{0}^{t} h X_{s} d s+W_{t}(h \neq 0),
\end{gathered}
$$

where $V, W$ are two independent standard Brownian motions, $X_{0}$ is a random variable in $\mathbb{R}$, of law $\pi_{0}$. We set $\left(\mathcal{F}_{t}\right)_{t \geq 0}$ to be the filtration associated to $\left(V_{t}, W_{t}\right)$. For $t \geq 0$, we call optimal filter at time $t$ the law of $X_{t}$ knowing $\left(Y_{s}\right)_{0 \leq s \leq t}$, and we denote this law by $\pi_{t}$. Let $\tau>1$, this parameter will be adjusted later. For any $t>0$, we set $Q_{t}$ to be the transition kernel of the Markov chain $\left(X_{k t}\right)_{k \geq 0}$. We set $Q=Q_{\tau}$.

Before going further, we mention that we believe the results we have could be transposed to processes in $\mathbb{R}^{d}$ for any $d$. This would result in a more technical paper and we chose not to pursue this goal.

Hypothesis 1. We suppose that $f$ is $C^{1}$ and that $\|f\|_{\infty},\left\|f^{\prime}\right\|_{\infty}$ are bounded by a constant $M$.

Hypothesis 2. We suppose that there exists positive constants $v_{1}, v_{2}$ such that, for all nonnegative $\Delta$,

$$
\pi_{0}\left([-\Delta, \Delta]^{\complement}\right) \leq v_{1} e^{-v_{2} \Delta^{2}} .
$$

(This means that the tail of $\pi_{0}$ is sub-Gaussian.) 
We are interested in the stability of $\left(\pi_{t}\right)_{t \geq 0}$ with respect to its initial condition. As explained below in Equation (1.2), for all $t, \pi_{t}$ can be written as a functional of $\left(Y_{s}\right)_{0 \leq s \leq t}$ and $\pi_{0}$. Suppose now we plug a probability $\pi_{0}^{\prime}$ instead of $\pi_{0}$ into this functional, we then obtain what is called a "wrongly initialized filter" $\pi_{t}^{\prime}$ (Equation (1.3)). It is natural to wonder whether $\pi_{t}-\pi_{t}^{\prime}$ goes to zero when $t$ goes to infinity in any sense, and at which rate it does so. We would then say that the filter $\left(\pi_{t}\right)$ is stable with respect to its initial condition. We give such a result with a rate of convergence, in Theorem 1.2, under Hypothesis 1 and 2. This question has been answered for more general processes $\left(X_{t}\right)$ and $\left(Y_{t}\right)$ evolving in continuous time. Here is a brief review and the existing results. We stress the differences with our setting (see [CR11] for other references).

- There is a proof of stability with respect to the initial condition in [AZ97]. In this paper, the process $\left(X_{t}\right)$ has to stay in a compact space. The rate is exponential.

- There are stability results in [Ata98], [KV08]. There, the process $\left(X_{t}\right)$ has to satisfy some ergodicity conditions. The rate is exponential.

- In [BCL04, CvH07], the process $\left(X_{t}\right)$ has to take values in a finite state space.

- The article [Sta05] is the closest to our results. There are some assumptions on the drift coefficient $f(\ldots)$ (see Section 1.3 of [Sta05], $f$ would have to be of the form $\varphi^{\prime} / \varphi, \varphi \in \mathcal{C}^{2}(\mathbb{R})$ ), which are different from ours. The rate is exponential.

In the case of an exponential rate, the filter would be called "exponentially stable" (with respect to its initial condition). The widespread idea is that exponential stability induces that a numerical approximation of the optimal filter would not deteriorate in time. Such an approximation is usually based on a time-recursive computation and it is believed that exponential stability will prevent an accumulation of errors. In order to use a stability result in a proof concerning a numerical scheme, one might like the distance between $\pi_{t}$ and $\pi_{t}^{\prime}$ to be expressed in term of the distance between $\pi_{0}$ and $\pi_{0}^{\prime}$, and there is no such result, at least when the time is continuous. We do not prove such a result in this paper. We explain below that there is another way to prove that a numerical approximation of the optimal filter does not deteriorate in time.

Again, our aim in this paper is to show stability in such a way that the results can be used in a proof that a numerical scheme remains good uniformly in time. This has not been done in the literature we cited above. We follow [OR05] by introducing a "robust filter" restricted to compact spaces. We show that this filter remains close to the optimal filter uniformly in time and this is enough to prove the stability of the optimal filter with respect to its initial condition. As in [OR05], we do not show that the optimal filter is exponentially stable, nor can we write the dependency in $\pi_{0}, \pi_{0}^{\prime}$ in the stability result. However, in a future work, we will use the stability properties of the robust filter to show that there exists a numerical approximation that remains uniformly good in time.

In the case where $f$ satisfies

$$
f^{\prime}(x)+f(x)^{2}+h^{2} x^{2}=P(x)
$$

where $P(x)$ is a second-order polynomial with positive leading-order coefficient, then $\pi_{t}$ is called the Beneš filter (see [Ben81], [BC09]) and there exists an explicit formula for the density of $\pi_{t}$, for all $t$. The study of the Beneš filter is developed in [Oco99]. Under Hypothesis 1, we have (1.1) if and only if $f(x)=f(0)(1+x f(0))^{-1}$. What we present here is a case in the neighborhood of the Beneš filter.

We present the main results and our strategy in Section 1.2 below. Before this, we have to go through some definitions.

For all $t>0$, the law of $Y_{0: t}$ under $\mathbb{P}$ and conditionally on $X_{0}, X_{t}$ has a density denoted by $y_{0: t} \mapsto \psi_{t}\left(y_{0: t}, X_{0}, X_{\tau}\right)$ with respect to the Wiener measure (see Lemma 2.2 below). The Kallianpur-Striebel formula (see [BC09], p. 57) gives us the following result (see the proof in Section 7.1). 
Lemma 1.1. For all $t>0$ and all bounded continuous $\varphi$,

$$
\pi_{t}(\varphi)=\frac{\int_{\mathbb{R}} \varphi(y) Q_{t}(x, d y) \psi_{t}\left(Y_{0: t}, x, y\right) \pi_{0}(d x)}{\int_{\mathbb{R}} Q_{t}(x, d y) \psi_{t}\left(Y_{0: t}, x, y\right) \pi_{0}(d x)}
$$

For any probability law $\pi_{0}^{\prime}$ on $\mathbb{R}$, we define the wrongly initialized filter (with initial condition $\left.\pi_{0}^{\prime}\right)$ by, for any $t>0$,

$$
\pi_{t}^{\prime}(\varphi)=\frac{\int_{\mathbb{R}} \varphi(y) Q_{t}(x, d y) \psi_{t}\left(Y_{0: t}, x, y\right) \pi_{0}^{\prime}(d x)}{\int_{\mathbb{R}} Q_{t}(x, d y) \psi_{t}\left(Y_{0: t}, x, y\right) \pi_{0}^{\prime}(d x)}
$$

\subsection{Main results and outline of the proofs}

All the results are given under Hypothesis 1 and 2. The first main result is the following $(\|\ldots\|$ is the total variation norm when applied to measures).

Theorem 1.2. There exists $\nu_{0}>0$ such that, if $\pi_{0}$ and $\pi_{0}^{\prime}$ are comparable,

$$
\mathbb{E}\left(\left\|\pi_{t}-\pi_{t}^{\prime}\right\|\right)=O\left(t^{-\nu_{0}}\right),
$$

when $t \rightarrow+\infty$ (see Section 3.1 for the definition of comparable).

Remark 1.3. We have here a polynomial stability whereas filters in discrete time are usually exponentially stable. We believe this result is not sharp (mainly because the proof is very convoluted).

In order to prove this Theorem, we first reduce the problem to a problem in discrete time by looking at the sequences $\left(\pi_{0}, \pi_{\tau}, \pi_{2 \tau}, \ldots\right),\left(\pi_{0}^{\prime}, \pi_{\tau}^{\prime}, \pi_{2 \tau}^{\prime}, \ldots\right)$. We then introduce a truncated filter $\left(\pi_{k \tau}^{\Delta}\right)_{k \geq 0}$ and a wrongly initialized truncated filter $\left(\left(\pi^{\prime}\right)_{k \tau}^{\Delta}\right)_{k \geq 0}$, where $\Delta$ is a truncation parameter. We do not get into more details since to get a closed definition of the truncated filter, one would have to look at Equations (4.29), (4.28), (3.1), (4.25), (4.19), (4.20), (4.26), (4.3), (7.19), (7.4), (7.21), (2.6), (2.7), (2.8), (2.5), (2.10), (2.13) and Lemma 2.3. The truncated filter can be viewed as a restriction of $\left(\pi_{k \tau}\right)_{k \geq 0}$ to some compacts. Equation (4.29) tells us that

$$
\pi_{k \tau}^{\Delta}=\bar{R}_{k}^{\Delta} \bar{R}_{k-1}^{\Delta} \ldots \bar{R}_{1}^{\Delta}\left(\pi_{0}\right), \text { for all } k \geq 1,
$$

for some operators $\bar{R}_{\ldots}^{\Delta}$. We define

$$
\left(\pi^{\prime}\right)_{k \tau}^{\Delta}=\bar{R}_{k}^{\Delta} \bar{R}_{k-1}^{\Delta} \ldots \bar{R}_{1}^{\Delta}\left(\pi_{0}^{\prime}\right), \text { for all } k \geq 1 .
$$

We first prove that the operators $\bar{R}_{\ldots}^{\Delta}$ are contracting (see Section 5, Equations (5.3), (5.8) for the definitions of $\left.\epsilon_{\ldots}, \epsilon_{\ldots}^{\prime}\right)$.

Lemma 1.4. We suppose that $\tau$ and $h$ are chose such that Equation (4.4) holds. For $n \geq 1$ and $k$ in $\{1,2, \ldots, n-1\}$, for all $\mu, \mu^{\prime}$ in $\mathcal{P}(\mathbb{R})$,

$$
\begin{aligned}
\left\|\bar{R}_{n}^{\Delta} \bar{R}_{n-1}^{\Delta} \ldots \bar{R}_{k+1}^{\Delta}(\mu)-\bar{R}_{n}^{\Delta} \bar{R}_{n-1}^{\Delta} \ldots \bar{R}_{k+1}^{\Delta}\left(\mu^{\prime}\right)\right\| & \\
\leq & \prod_{i=1}^{\lfloor(n-k) / 2\rfloor}\left(1-\epsilon_{k+2 i-1}^{2}\left(\epsilon_{k+2 i}^{\prime}\right)^{2}\right) \times 4 \inf \left(1, \frac{\left\|\mu-\mu^{\prime}\right\|}{\left(\epsilon_{k+2}^{\prime}\right)^{2} \epsilon_{k+1}^{4}}\right) .
\end{aligned}
$$

We then write a telescopic sum (Equation (6.2)) for the optimal filter

$$
\left\|\pi_{n \tau}-\pi_{n \tau}^{\Delta}\right\| \leq\left\|\pi_{n \tau}-\bar{R}_{n}^{\Delta}\left(\pi_{(n-1) \tau}\right)\right\|+\sum_{1 \leq k \leq n-1}\left\|\bar{R}_{n: k+1}^{\Delta}\left(\pi_{k \tau}\right)-\bar{R}_{n: k+1}^{\Delta}\left(\bar{R}_{k}^{\Delta}\left(\pi_{(k-1) \tau}\right)\right)\right\|
$$

where, for all $n \geq k, \bar{R}_{n: k+1}^{\Delta}=\bar{R}_{n}^{\Delta} \circ \bar{R}_{n-1}^{\Delta} \circ \cdots \circ \bar{R}_{k+1}^{\Delta}$ is a composition of operators (and we write a similar sum for the wrongly initialized filter). Due to the contractivity of the operators $\bar{R}_{\ldots}^{\Delta}$, we are then able to prove that the truncation error is uniform in time (see Section 2.5 for the definition of $\preceq$ ). 
Proposition 1.5. There exist a function of $h, \lambda_{1}^{\prime}(h)$ and a constant $\tau_{\infty}$ such that, if $\tau \geq \tau_{\infty}$, we have

$$
\sup _{n \geq 0} \log \left(\mathbb{E}\left(\left\|\pi_{n \tau}-\pi_{n \tau}^{\Delta}\right\|\right)\right) \underset{\Delta, c}{\preceq}-\Delta^{2} \times \lambda_{1}^{\prime}(h) ;
$$

and, if $\pi_{0}$ and $\pi_{0}^{\prime}$ are comparable,

$$
\sup _{n \geq 0} \log \left(\mathbb{E}\left(\left\|\pi_{n \tau}^{\prime}-\left(\pi^{\prime}\right)_{n \tau}^{\Delta}\right\|\right)\right) \underset{\Delta, c}{\preceq}-\Delta^{2} \times \lambda_{1}^{\prime}(h) .
$$

(See Section 3.1 for a definition of comparable.)

The proof of Theorem 1.2 then start with the inequality

$$
\left\|\pi_{n \tau}-\pi_{n \tau}^{\prime}\right\| \leq\left\|\pi_{n \tau}-\pi_{n \tau}^{\Delta}\right\|+\left\|\pi_{n \tau}^{\Delta}-\left(\pi^{\prime}\right)_{n \tau}^{\Delta}\right\|+\left\|\left(\pi^{\prime}\right)_{n \tau}^{\Delta}-\pi_{n \tau}^{\prime}\right\| .
$$

We choose $\Delta$ as a function of $n$ to make sure all the terms on the right go to zero when $n$ goes to infinity. After some technicalities, we are able to get rid of the discrete time index $n \tau$ and write a result for any $t \geq 0$.

The second main result of the paper is that these results can be used to show that a numerical approximation of the filter does not deteriorate in time. Suppose we have a numerical approximation $\pi_{n \tau}^{N, \Delta}$ of $\pi_{n \tau}^{\Delta}$ for all $n \geq 0$ (with $\pi_{0}^{N, \Delta}=\pi_{0}^{\Delta}$ ); $\Delta$ is again the truncation parameter and $N$ is the approximation parameter (one can think of a number of particles in case of a particle filter approximation). We write

$$
\left\|\pi_{n \tau}-\pi_{n \tau}^{N, \Delta}\right\| \leq\left\|\pi_{n \tau}-\pi_{n \tau}^{\Delta}\right\|+\left\|\pi_{n \tau}^{\Delta}-\pi_{n \tau}^{N, \Delta}\right\|, \text { for all } n \geq 0 .
$$

We already have the result that $\left\|\pi_{n \tau}-\pi_{n \tau}^{\Delta}\right\|$ is small when $\Delta$ goes to infinity (Proposition 1.5). For the second term, we write a telescopic sum

$$
\left\|\pi_{n \tau}^{N, \Delta}-\pi_{n \tau}^{\Delta}\right\| \leq\left\|\pi_{n \tau}^{N, \Delta}-\bar{R}_{n}^{\Delta}\left(\pi_{(n-1) \tau}^{N, \Delta}\right)\right\|+\sum_{1 \leq k \leq n-1}\left\|\bar{R}_{n: k+1}^{\Delta}\left(\pi_{k \tau}^{N, \Delta}\right)-\bar{R}_{n: k+1}^{\Delta}\left(\bar{R}_{k}^{\Delta}\left(\pi_{(k-1) \tau}^{N, \Delta}\right)\right)\right\| .
$$

Using Lemma 1.4 again, we would be able to show that $\left\|\pi_{n \tau}^{N, \Delta}-\pi_{n \tau}^{\Delta}\right\|$ remains small uniformly in $n$ (when $N$ is big). Choosing $\Delta$ as a function of $N_{-}$in the same way as in [OR05] _ would then allow to show that $\left\|\pi_{n \tau}-\pi_{n \tau}^{N, \Delta}\right\|$ remains small uniformly in the time $n$, when $N$ is big.

The fact that stability results have the potential to be used to show that a numerical approximation does not deteriorate in time is a novelty compared to the other papers in the area.

Another novelty lies in the proof of Lemma 1.4. In Proposition 5.6, we exhibit a representation of the truncated filter $\bar{R}_{n: 1}^{\Delta}\left(\pi_{0}\right)$ as the $n$-th point of a Feynman-Kac sequence based on a mixing Markov kernel (see Section 3 for the definition of the term Feynman-Kac sequence). This is not straightforward because the observations are given as a process in continuous time. Whatever transformation we adopt, the problem remains more difficult than in discrete time. We will write more comments about the novelty of the proof in Section 5 (it requires more notations to develop on the subject).

The outline of the paper is as follows. In Section 2, we compute useful estimates concerning $\psi_{t}$. In Section 3, we recall useful notions on filtering. In Section 4 and 5, we introduce the truncated filter and its properties. At the beginning of Section 5, we elaborate on our strategy. In Section 6, we prove that the optimal filter can be approximated by robust filters uniformly in time (Proposition 1.5), and that the optimal filter is stable with respect to its initial condition (Theorem 1.2).

\section{Computations around $\psi_{t}$}

\subsection{Estimation of the transition density and the likelihood}

We begin by bounding the transition density by above and below (see the proof in Section 7.1). 
Lemma 2.1. For all $x, y \in \mathbb{R}, Q(x, d y)$ has a density $Q(x, y)$ with respect to the Lebesgue measure and

$$
\frac{e^{-\frac{(y-x)^{2}}{2 \tau}}}{\sqrt{2 \pi \tau}} e^{-M|y-x|-\tau\left(\frac{M}{2}+\frac{M^{2}}{2}\right)} \leq Q(x, y) \leq \frac{\exp ^{-\frac{(y-x)^{2}}{2 \tau}}}{\sqrt{2 \pi \tau}} e^{M|y-x|+\frac{M \tau}{2}} .
$$

Following [BC09] (Chapter 6, Section 6.1), we define a new probability $\widehat{\mathbb{P}}$ by (for all $t \geq 0$ )

$$
\begin{gathered}
\widehat{V}_{t}=V_{t}+\int_{0}^{t} f\left(X_{s}\right) d s \\
\left.\frac{d \mathbb{P}}{d \widehat{\mathbb{P}}}\right|_{\mathcal{F}_{t}}=\widehat{Z}_{t}=\exp \left(\int_{0}^{t} f\left(X_{s}\right) d \widehat{V}_{s}-\frac{1}{2} \int_{0}^{t} f\left(X_{s}\right)^{2} d s+\int_{0}^{t} h X_{s} d Y_{s}-\frac{1}{2} \int_{0}^{t} h^{2} X_{s}^{2} d s\right)
\end{gathered}
$$

We define, for all $0 \leq s \leq t$,

$$
Y_{s: t}=\left(Y_{u}\right)_{s \leq u \leq t}
$$

We set

$$
\widehat{\psi}_{t}\left(y_{0: t}, x_{0}, x_{1}\right)=\mathbb{E}^{\widehat{\mathbb{P}}}\left(\exp \left(\int_{0}^{t} h X_{s} d Y_{s}-\frac{1}{2} \int_{0}^{t}\left(h X_{s}\right)^{2} d s\right) \mid X_{0}=x_{0}, X_{t}=x_{1}, Y_{0: t}=y_{0: t}\right) .
$$

We set

$$
\psi=\psi_{\tau}, \widehat{\psi}=\widehat{\psi}_{\tau}
$$

We have the following bounds for the likelihood (see the proof in Section 7.1).

Lemma 2.2. For all $t>0$, the law of $Y_{0: t}$ under $\mathbb{P}$ and conditionally on $X_{0}, X_{t}$ has a density denoted by $y_{0: t} \mapsto \psi_{t}\left(y_{0: t}, X_{0}, X_{\tau}\right)$ with respect to the Wiener measure. This density satisfies, for all $x, z \in \mathbb{R}$ and any continuous trajectory $y_{0: t}$

$$
\widehat{\psi}_{t}\left(y_{0: t}, x, z\right) e^{-2 M|z-x|-\tau\left(M+\frac{M^{2}}{2}\right)} \leq \psi_{t}\left(y_{0: t}, x, z\right) \leq e^{2 M|z-x|+\tau\left(M+\frac{M^{2}}{2}\right)} \widehat{\psi}_{t}\left(y_{0: t}, x, z\right)
$$

\subsection{Change of measure}

Under $\widehat{\mathbb{P}}, \widehat{V}$ is a standard Brownian motion. So, using a standard representation of a Brownian bridge, we can rewrite $\widehat{\psi}$ as

$$
\begin{aligned}
\widehat{\psi}\left(y_{0: \tau}, x, z\right)=\mathbb{E}\left(\operatorname { e x p } \left(\int _ { 0 } ^ { \tau } h \left(x\left(1-\frac{s}{\tau}\right)\right.\right.\right. & \left.+z \frac{s}{\tau}+\left(B_{s}-\frac{s}{\tau} B_{\tau}\right)\right) d y_{s} \\
& \left.\left.-\frac{h^{2}}{2} \int_{0}^{\tau}\left(x\left(1-\frac{s}{\tau}\right)+z \frac{s}{\tau}+\left(B_{s}-\frac{s}{\tau} B_{\tau}\right)\right)^{2} d s\right)\right)
\end{aligned}
$$

where $B$ is a standard Brownian motion (under $\mathbb{P}$ ). As we want to compute the above integral, where $B$ is the only random variable involved, we can suppose that $B$ is adapted to the filtration $\mathcal{F}$. We have (using the change of variable $s^{\prime}=s / \tau$ and the scaling property of the Brownian motion)

$$
\begin{aligned}
& \widehat{\psi}\left(y_{0: \tau}, x, z\right)=\mathbb{E}( \exp \left(\int_{0}^{1} h\left(x\left(1-s^{\prime}\right)+z s^{\prime}+B_{\tau s^{\prime}}-s^{\prime} B_{\tau}\right) d y_{\tau s^{\prime}}\right. \\
&\left.\left.-\frac{h^{2} \tau}{2} \int_{0}^{1}\left(x\left(1-s^{\prime}\right)+z s^{\prime}+B_{\tau s^{\prime}}-s^{\prime} B_{\tau}\right)^{2} d s^{\prime}\right)\right) \\
&=\mathbb{E}\left(\operatorname { e x p } \left(\int_{0}^{1} h\left(x\left(1-s^{\prime}\right)+z s^{\prime}+\sqrt{\tau}\left(B_{s^{\prime}}-s^{\prime} B_{1}\right)\right) d y_{\tau s^{\prime}}\right.\right.
\end{aligned}
$$




$$
\left.\left.-\frac{h^{2} \tau}{2} \int_{0}^{1}\left(x\left(1-s^{\prime}\right)+z s^{\prime}+\sqrt{\tau}\left(B_{s^{\prime}}-s^{\prime} B_{1}\right)\right)^{2} d s^{\prime}\right)\right)
$$

In the spirit of [MY08] (Section 2.1), we define a new probability $\mathbb{Q}$ by (for all $t$ )

$$
\left.\frac{d \mathbb{Q}}{d \mathbb{P}}\right|_{\mathcal{F}_{t}}=\exp \left(-\frac{h^{2} \tau^{2}}{2} \int_{0}^{1} B_{s}^{2} d s-h \tau \int_{0}^{1} B_{s} d B_{s}\right) .
$$

By Girsanov's theorem, under the probability $\mathbb{Q}$, the process

$$
\beta_{t}=B_{t}+\int_{0}^{t} h \tau B_{s} d s, \forall t \geq 0
$$

is a Brownian motion. We get

$$
\begin{array}{r}
\widehat{\psi}\left(y_{0: \tau}, x, z\right)=\exp \left(\int_{0}^{1} h(x(1-s)+z s) d y_{\tau s}-\frac{h^{2} \tau}{2} \int_{0}^{1}(x(1-s)+z s)^{2} d s\right) \\
\times \mathbb{E}^{\mathbb{Q}}\left(\operatorname { e x p } \left(\int_{0}^{1} h \sqrt{\tau}\left(B_{s}-s B_{1}\right) d y_{\tau s}-h^{2} \tau^{3 / 2} \int_{0}^{1}(x(1-s)+z s)\left(B_{s}-s B_{1}\right) d s\right.\right. \\
\left.\left.-\frac{h^{2} \tau^{2}}{2} \int_{0}^{1} s^{2} B_{1}^{2}-2 s B_{s} B_{1} d s+h \tau \int_{0}^{1} B_{s} d B_{s}\right)\right) .
\end{array}
$$

Using the integration by parts formula, we can rewrite the last expectation as

$$
\begin{aligned}
& \mathbb{E}^{\mathbb{Q}}\left(\operatorname { e x p } \left(-h \sqrt{\tau} \int_{0}^{1}\left(y_{\tau s}-\int_{0}^{1} y_{\tau u} d u\right) d B_{s}+h^{2} \tau^{3 / 2} \int_{0}^{1}\left(-x \frac{(1-s)^{2}}{2}+z \frac{s^{2}}{2}+\frac{x}{6}-\frac{z}{6}\right) d B_{s}\right.\right. \\
& \left.\left.+h^{2} \tau^{2} B_{1}\left(\frac{B_{1}}{3}-\int_{0}^{1} \frac{s^{2}}{2} d B_{s}\right)+h \tau\left(\frac{B_{1}^{2}}{2}-\frac{1}{2}\right)\right)\right)= \\
& \mathbb{E}^{\mathbb{Q}}\left(\operatorname { e x p } \left(-h \sqrt{\tau} \int_{0}^{1}\left(y_{\tau s}-\int_{0}^{1} y_{\tau u} d u\right) d B_{s}+h^{2} \tau^{3 / 2} x \int_{0}^{1} s d B_{s}+\frac{h^{2} \tau^{3 / 2}}{2}(z-x) \int_{0}^{1} s^{2} d B_{s}\right.\right. \\
& \left.\left.-h^{2} \tau^{3 / 2}\left(\frac{x}{3}+\frac{z}{6}\right) B_{1}+\left(\frac{h^{2} \tau^{2}}{3}+\frac{h \tau}{2}\right) B_{1}^{2}-\frac{h^{2} \tau^{2}}{2} B_{1} \int_{0}^{1} s^{2} d B_{s}-\frac{h \tau}{2}\right)\right) .
\end{aligned}
$$

\subsection{Covariances computation}

The last expectation contains an exponential of a polynomial of degree 2 of 4 Gaussians:

$$
G_{1}=B_{1}, G_{2}=\int_{0}^{1} s d B_{s}, G_{3}=\int_{0}^{1} s^{2} d B_{s}, G_{4}=\int_{0}^{1}\left(y_{\tau s}-\int_{0}^{1} y_{\tau u} d u\right) d B_{s} .
$$

So this expectation can be expressed as a function of the covariance matrix of these Gaussians. We compute here the covariances, which do not depend on $y_{0: \tau}$. We set

$$
\theta=h \tau .
$$

Lemma 2.3. We have:

$$
\begin{gathered}
\operatorname{Var}^{\mathbb{Q}}\left(G_{1}\right)=\frac{1-e^{-2 \theta}}{2 \theta}, \\
\operatorname{Var}^{\mathbb{Q}}\left(G_{2}\right)=\left(1+\frac{1}{\theta}\right)^{2} \frac{\left(1-e^{-2 \theta}\right)}{2 \theta}+\frac{1}{\theta^{2}}-\left(\frac{2}{\theta^{2}}+\frac{2}{\theta^{3}}\right)\left(1-e^{-\theta}\right), \\
\operatorname{Var}^{\mathbb{Q}}\left(G_{3}\right)=\left(1+\frac{2}{\theta}+\frac{2}{\theta^{2}}\right)^{2} \frac{\left(1-e^{-2 \theta}\right)}{2 \theta}+\left(\frac{2}{\theta}+\frac{2}{\theta^{2}}\right)^{3} \frac{\theta}{6}-\frac{8}{6 \theta^{5}}-\frac{4}{\theta^{2}}\left(1+\frac{2}{\theta}+\frac{2}{\theta^{2}}\right)
\end{gathered}
$$




$$
\begin{gathered}
\operatorname{Cov}^{\mathbb{Q}}\left(G_{1}, G_{2}\right)=\left(\frac{1}{2 \theta}+\frac{1}{2 \theta^{2}}\right)\left(1-e^{-2 \theta}\right)+\frac{e^{-\theta}-1}{\theta^{2}}, \\
\operatorname{Cov}^{\mathbb{Q}}\left(G_{1}, G_{3}\right)=\left(\frac{1}{2 \theta}+\frac{1}{\theta^{2}}+\frac{1}{\theta^{3}}\right)\left(1-e^{-2 \theta}\right)-\frac{2}{\theta^{2}}, \\
\operatorname{Cov}^{\mathbb{Q}}\left(G_{2}, G_{3}\right)=\left(1+\frac{1}{\theta}\right)\left(\frac{1}{2 \theta}+\frac{1}{\theta^{2}}+\frac{1}{\theta^{3}}\right)\left(1-e^{-2 \theta}\right)-\left(\frac{1}{\theta^{2}}+\frac{2}{\theta^{3}}+\frac{2}{\theta^{4}}\right)\left(1-e^{-\theta}\right)-\frac{1}{\theta^{2}} .
\end{gathered}
$$

See the proof in Section 7.2

Let $U_{1}, U_{2}, U_{3}, U_{4}$ be i.i.d. of law $\mathcal{N}(0,1)$. We can find $\alpha, \beta, \gamma, a, b, c, \lambda_{1}, \lambda_{2}, \lambda_{3}, \lambda_{4} \in \mathbb{R}$ such that (under $\mathbb{Q}$ )

$$
\left(\begin{array}{l}
G_{1} \\
G_{3} \\
G_{2} \\
G_{4}
\end{array}\right) \stackrel{\operatorname{law}}{=}\left(\begin{array}{cccc}
\alpha U_{1} & & & \\
\beta U_{1} & +\gamma U_{2} & & \\
a U_{1} & +b U_{2} & +c U_{3} & \\
\lambda_{1} U_{1} & +\lambda_{2} U_{2} & +\lambda_{3} U_{3} & +\lambda_{4} U_{4}
\end{array}\right) .
$$

(There is no mistake here, we do intend to look at the vector $\left(G_{1}, G_{3}, G_{2}, G_{4}\right)$.) Indeed, we take

$$
\begin{gathered}
\alpha=\sqrt{\operatorname{Var}^{\mathbb{Q}}\left(G_{1}\right)}, \beta=\frac{\operatorname{Cov}^{\mathbb{Q}}\left(G_{1}, G_{3}\right)}{\alpha}, \gamma=\sqrt{\operatorname{Var}^{\mathbb{Q}}\left(G_{3}\right)-\beta^{2}}, \\
a=\frac{\operatorname{Cov}^{\mathbb{Q}}\left(G_{1}, G_{2}\right)}{\alpha}, b=\frac{\operatorname{Cov}^{\mathbb{Q}}\left(G_{2}, G_{3}\right)-a \beta}{\gamma}, c=\sqrt{\operatorname{Var}^{\mathbb{Q}}\left(G_{2}\right)-a^{2}-b^{2}} .
\end{gathered}
$$

And we find $\lambda_{1}, \ldots, \lambda_{4}$ by solving

$$
\left\{\begin{array}{rlrl}
\alpha \lambda_{1} & & =\operatorname{Cov}^{\mathbb{Q}}\left(G_{1}, G_{4}\right) \\
\beta \lambda_{1}+\gamma \lambda_{2} & & =\operatorname{Cov}^{\mathbb{Q}}\left(G_{3}, G_{4}\right) \\
a \lambda_{1}+b \lambda_{2}+c \lambda_{3} & =\operatorname{Cov}^{\mathbb{Q}}\left(G_{2}, G_{4}\right) \\
\lambda_{1}^{2}+\lambda_{2}^{2}+\lambda_{3}^{2}+\lambda_{4}^{2} & =\operatorname{Var}^{\mathbb{Q}}\left(G_{4}\right) .
\end{array}\right.
$$

We observe that $\alpha, \beta, \gamma, a, b, c$ can be written explicitly in terms of the parameters of the problem.

\subsection{Integral computation}

The last part of (2.4) is equal to

$$
\begin{aligned}
& \mathbb{E}^{\mathbb{Q}}\left(\operatorname { e x p } \left(\left(\frac{\theta^{2}}{3}+\frac{\theta}{2}\right) G_{1}^{2}-\frac{\theta^{2}}{2} G_{1} G_{3}-h^{2} \tau^{3 / 2}\left(\frac{x}{3}+\frac{z}{6}\right) G_{1}\right.\right. \\
& \left.\left.\left.+h^{2} \tau^{3 / 2} x G_{2}+\frac{h^{2} \tau^{3 / 2}}{2}(z-x) G_{3}-h \sqrt{\tau} G_{4}-\frac{\theta}{2}\right)\right)\right)= \\
& \int_{u_{1, \ldots, u_{4} \in \mathbb{R}}} \exp \left(\left(\frac{\theta^{2}}{3}+\frac{\theta}{2}\right) \alpha^{2} u_{1}^{2}-\frac{\theta^{2}}{2} \alpha u_{1}\left(\beta u_{1}+\gamma u_{2}\right)-h^{2} \tau^{3 / 2}\left(\frac{x}{3}+\frac{z}{6}\right) \alpha u_{1}\right. \\
& \left.+h^{2} \tau^{3 / 2} x\left(a u_{1}+b u_{2}+c u_{3}\right)+\frac{h^{2} \tau^{3 / 2}}{2}(z-x)\left(\beta u_{1}+\gamma u_{2}\right)-h \sqrt{\tau}\left(\lambda_{1} u_{1}+\cdots+\lambda_{4} u_{4}\right)-\frac{\theta}{2}\right) \\
& \frac{\exp \left(-\frac{\left(u_{1}^{2}+\cdots+u_{4}^{2}\right)}{2}\right)}{(2 \pi)^{2}} d u_{1} \ldots d u_{4}= \\
& \int_{u_{1}, \ldots, u_{4} \in \mathbb{R}} \exp \left\{-\frac{1}{2 \sigma_{1}^{2}}\left[u_{1}-\sigma_{1}^{2}\left(-\frac{\theta^{2} \alpha \gamma}{2} u_{2}-h^{2} \tau^{3 / 2}\left(\frac{x}{3}+\frac{z}{6}\right) \alpha+h^{2} \tau^{3 / 2} x a\right.\right.\right. \\
& \left.\left.+\frac{h^{2} \tau^{3 / 2}}{2}(z-x) \beta-h \sqrt{\tau} \lambda_{1}\right)\right]^{2} \\
& +\frac{\sigma_{1}^{2}}{2}\left[-\frac{\theta^{2} \alpha \gamma}{2} u_{2}-h^{2} \tau^{3 / 2}\left(\frac{x}{3}+\frac{z}{6}\right) \alpha+h^{2} \tau^{3 / 2} x a+\frac{h^{2} \tau^{3 / 2}}{2}(z-x) \beta-h \sqrt{\tau} \lambda_{1}\right]^{2}
\end{aligned}
$$




$$
\begin{aligned}
+h^{2} \tau^{3 / 2} x\left(b u_{2}+c u_{3}\right)+\frac{h^{2} \tau^{3 / 2}}{2}(z-x) \gamma u_{2}-h \sqrt{\tau}\left(\lambda_{2} u_{2}+\cdots+\lambda_{4} u_{4}\right)-\frac{\theta}{2} \\
\left.-\frac{\left(u_{2}^{2}+\cdots+u_{4}^{2}\right)}{2}\right\} \frac{1}{(2 \pi)^{2}} d u_{1} \ldots d u_{4},
\end{aligned}
$$

where

$$
\sigma_{1}^{2}=\left(2\left(-\left(\frac{\theta^{2}}{3}+\frac{\theta}{2}\right) \alpha^{2}+\frac{\theta^{2} \alpha \beta}{2}+\frac{1}{2}\right)\right)^{-1} .
$$

As the above expectation is finite then $\sigma_{1}^{2}$ is well defined. We set

$$
m_{1}=\sigma_{1}^{2}\left(-h^{2} \tau^{3 / 2}\left(\frac{x}{3}+\frac{z}{6}\right) \alpha+h^{2} \tau^{3 / 2} x a+\frac{h^{2} \tau^{3 / 2}}{2}(z-x) \beta-h \sqrt{\tau} \lambda_{1}\right) .
$$

The above expectation (2.9) is equal to:

$$
\begin{gathered}
\int_{u_{1, \ldots, u_{4} \in \mathbb{R}} \exp (-} \frac{1}{2 \sigma_{1}^{2}}\left[u_{1}+\sigma_{1}^{2} \frac{\theta^{2} \alpha \gamma}{2} u_{2}-m_{1}\right]^{2} \\
+\left(\frac{\sigma_{1}^{2} \theta^{2} \alpha \gamma}{2}\right)^{2} u_{2}^{2} \frac{1}{2 \sigma_{1}^{2}}+\frac{m_{1}^{2}}{2 \sigma_{1}^{2}}-\frac{1}{2 \sigma_{1}^{2}} \times 2\left(\frac{\sigma_{1}^{2} \theta^{2} \alpha \gamma}{2}\right) m_{1} u_{2} \\
\quad+h^{2} \tau^{3 / 2} x\left(b u_{2}+c u_{3}\right)+\frac{h^{2} \tau^{3 / 2}}{2}(z-x) \gamma u_{2} \\
\left.-h \sqrt{\tau}\left(\lambda_{2} u_{2}+\lambda_{3} u_{3}+\lambda_{4} u_{4}\right)-\frac{\left(u_{2}^{2}+u_{3}^{2}+u_{4}^{2}\right)}{2}-\frac{\theta}{2}\right) \frac{1}{(2 \pi)^{2}} d u_{1} \ldots d u_{4}= \\
\quad \int_{u_{1}, \ldots, u_{4} \in \mathbb{R}} \exp \left(-\frac{1}{2 \sigma_{1}^{2}}\left[u_{1}+\sigma_{1}^{2} \frac{\theta^{2} \alpha \gamma}{2} u_{2}-m_{1}\right]^{2}\right. \\
-\frac{1}{2 \sigma_{2}^{2}}\left[u_{2}-\sigma_{2}^{2}\left(h^{2} \tau^{3 / 2} x b+\frac{h^{2} \tau^{3 / 2}}{2}(z-x) \gamma-h \sqrt{\tau} \lambda_{2}-\frac{\theta^{2} \alpha \gamma m_{1}}{2}\right)\right]^{2} \\
\left.+\frac{m_{2}^{2}}{2 \sigma_{2}^{2}}+\frac{m_{1}^{2}}{2 \sigma_{1}^{2}}+h^{2} \tau^{3 / 2} x c u_{3}-h \sqrt{\tau}\left(\lambda_{3} u_{3}+\lambda_{4} u_{4}\right)-\frac{\left(u_{3}^{2}+u_{4}^{2}\right)}{2}-\frac{\theta}{2}\right) \frac{1}{(2 \pi)^{2}},
\end{gathered}
$$

where

$$
\sigma_{2}^{2}=\left(2\left(-\frac{\sigma_{1}^{2} \theta^{4} \alpha^{2} \gamma^{2}}{8}+\frac{1}{2}\right)\right)^{-1}
$$

and

$$
m_{2}=\sigma_{2}^{2}\left(h^{2} \tau^{3 / 2} x b+\frac{h^{2} \tau^{3 / 2}}{2}(z-x) \gamma-h \sqrt{\tau} \lambda_{2}-\frac{\theta^{2} \alpha \gamma}{2} m_{1}\right) .
$$

Then (2.12) is equal to:

$$
\begin{gathered}
\int_{u_{1}, \ldots, u_{4} \in \mathbb{R}} \exp \left(-\frac{1}{2 \sigma_{1}^{2}}\left[u_{1}+\sigma_{1}^{2} \frac{\theta^{2} \alpha \gamma}{2} u_{2}-m_{1}\right]^{2}-\frac{1}{2 \sigma_{2}^{2}}\left[u_{2}-m_{2}\right]^{2}+\frac{m_{2}^{2}}{2 \sigma_{2}^{2}}+\frac{m_{1}^{2}}{2 \sigma_{1}^{2}}\right. \\
-\frac{1}{2}\left[u_{3}-h^{2} \tau^{3 / 2} c x+h \sqrt{\tau} \lambda_{3}\right]^{2}-\frac{1}{2}\left[u_{4}+h \sqrt{\tau} \lambda_{4}\right]^{2} \\
\left.+\frac{1}{2}\left(-h^{2} \tau^{3 / 2} c x+h \sqrt{\tau} \lambda_{3}\right)^{2}+\frac{1}{2}\left(-h \sqrt{\tau} \lambda_{4}\right)^{2}-\frac{\theta}{2}\right) \frac{1}{(2 \pi)^{2}} d u_{1} \ldots d u_{4}= \\
\sigma_{1} \sigma_{2} \exp \left(\frac{m_{1}^{2}}{2 \sigma_{1}^{2}}+\frac{m_{2}^{2}}{2 \sigma_{2}^{2}}+\frac{1}{2}\left(-h^{2} \tau^{3 / 2} c x+h \sqrt{\tau} \lambda_{3}\right)^{2}+\frac{1}{2}\left(h \sqrt{\tau} \lambda_{4}\right)^{2}-\frac{\theta}{2}\right)
\end{gathered}
$$




\subsection{Asymptotic $\tau \rightarrow+\infty$}

From $(2.3),(2.4),(2.9),(2.12),(2.15)$, we see that $\widehat{\psi}\left(y_{0: \tau}, x, z\right) \propto \exp (P(x, z))$ with $P$ a polynomial of degree 2 in $x, z$. Let us write $-A_{2}(\theta)$ for the coefficient of $x^{2}$ in $P,-B_{2}(\theta)$ for the coefficient of $z^{2}$ in $P, C_{1}(\theta)$ for the coefficient of $x z$ in $P, A_{1}(\theta)$ for the coefficient of $x$ in $P, B_{1}(\theta)$ for the coefficient of $z$ in $P$ and $C_{0}(\theta)$ for the "constant" coefficient. We will write $A_{1}^{y_{0: \tau}}(\theta)=A_{1}\left(y_{0: \tau}, \theta\right)$ (or simply $A_{1}^{y_{0: \tau}}$ ), etc, when in want of stressing the dependency in $y$. When there will be no ambiguity, we will drop the $y$. superscript. The coefficients $A_{2}^{y_{0: \tau}}, B_{2}^{y_{0: \tau}}, C_{1}^{y_{0: \tau}}$ do not depend on $y$ as it will be seen below. We have

$$
\widehat{\psi}\left(y_{0: \tau}, x, z\right)=\sigma_{1} \sigma_{2} \exp \left(-A_{2} x^{2}-B_{2} z^{2}+A_{1}^{y_{0: \tau}} x+B_{1}^{y_{0: \tau}} z+C_{1}^{y_{0: \tau}} x z+C_{0}^{y_{0: \tau}}\right) .
$$

We are interested in the limit $\tau \rightarrow+\infty$, with $h$ being fixed (or equivalently $\theta \rightarrow+\infty$ with $h$ being fixed).

Lemma 2.4. We have

$$
A_{2}(\theta) \underset{\theta \rightarrow+\infty}{\longrightarrow} \frac{h}{2}, B_{2}(\theta) \underset{\theta \rightarrow+\infty}{\longrightarrow} \frac{h}{2}, C_{1}(\theta)=-\frac{3 h}{2 \theta}+o\left(\frac{1}{\theta}\right) .
$$

The proof relies partly on a symbolic computation program (see Section 7.2).

Let us set, for all $s \leq t$,

$$
\mathcal{W}_{s, t}=\sup _{\left(s_{1}, s_{2}\right) \in[s, t]}\left|W_{s_{1}}-W_{s_{2}}\right|, \mathcal{V}_{s, t}=\sup _{\left(s_{1}, s_{2}\right) \in[s, t]}\left|V_{s_{1}}-V_{s_{2}}\right|
$$

Definition 2.5. Suppose we have functions $f_{1}, f_{2}$ going from some set $F$ to $\mathbb{R}$. We write

$$
f_{1} \preceq f_{2}
$$

if there exists a constant $B$ in $\mathbb{R}_{+}$, which does not depend on the parameters of our problem, such that $f_{1}(z) \leq B f_{2}(z)$, for all $z$ in $F$.

In the particular case when we are dealing with functions of a parameter $\Delta \in \mathbb{R}$, we write

$$
f_{1} \frac{\precsim}{\Delta} f_{2} \text { or } f_{1}(\Delta) \underset{\Delta}{\Delta} f_{2}(\Delta)
$$

if there exists a constant $B_{1}$ in $\mathbb{R}_{+}$, which does not depend on the parameters of our problem, and a constant $\Delta_{0}$, which may depend on the parameters of our problem, such that

$$
\Delta \geq \Delta_{0} \Rightarrow f_{1}(\Delta) \leq B_{1} f_{2}(\Delta)
$$

If, in addition, $\Delta_{0}$ depends continuously on the parameter $\tau$, we write

$$
f_{1} \underset{\Delta, c}{\preceq} f_{2}
$$

The notation $\preceq$ is akin to the notation $O(\ldots)$. It has the advantage that one can single out which asymptotic we are studying.

We state here (without proof) useful properties concerning the above Definition.

Lemma 2.6. Suppose we have functions $f, f_{1}, f_{2}, h_{1}, h_{2}$.

1. If $f \leq f_{1}+f_{2}$ and $f_{1} \preceq f_{2}$ then $f \preceq f_{2}$.

2. If $f \leq f_{1}+f_{2}$ and $\log \left(f_{1}\right) \underset{\Delta}{\preceq} h_{1}$ and $\log \left(f_{2}\right) \underset{\Delta}{\preceq} h_{2}$, with $h_{1}(\Delta), h_{2}(\Delta) \underset{\Delta \rightarrow \infty}{\longrightarrow}-\infty$, then $\log (f) \underset{\Delta}{\Delta} \sup \left(h_{1}, h_{2}\right)$. 
3. If we have $f_{1} \underset{\Delta, c}{\preceq} f_{2}$, say for $\tau \geq \tau_{0}\left(\tau_{0}>0\right)$, then there exists a constant $B_{1}$ and a continuous function $\Delta_{0}$ such that, for all $\tau \geq \tau_{0}$ and $\Delta \geq \Delta_{0}(\tau), f_{1}(\Delta) \leq B_{1} f_{2}(\Delta)$. In particular, for any $\tau_{1}>\tau_{0}$, if $\tau \in\left[\tau_{0}, \tau_{1}\right]$ and $\Delta \geq \sup _{t \in\left[\tau_{0}, \tau_{1}\right]} \Delta_{0}(t)$ then $f_{1}(\Delta) \leq B_{1} f_{2}(\Delta)$.

We have the following "variational" bounds on $B_{1}$ (see the proof in Section 7.2).

Lemma 2.7. For all $k \in \mathbb{N}$,

$$
\left|B_{1}\left(Y_{k \tau:(k+1) \tau}, \theta\right)-B_{1}\left(Y_{(k+1) \tau:(k+2) \tau}, \theta\right)\right| \preceq M h \tau+h \mathcal{V}_{k \tau,(k+2) \tau}+\left(h+\frac{1}{\tau}\right) \mathcal{W}_{k \tau,(k+2) \tau},
$$

and

$$
\left|B_{1}\left(Y_{0: \tau}, \theta\right)\right| \preceq M h \tau+h \mathcal{V}_{0,2 \tau}+\left(h+\frac{1}{\tau}\right) \mathcal{W}_{0,2 \tau}
$$

\section{Definitions and useful notions}

We follow here the ideas of [OR05].

\subsection{Notations}

We state here notations and definitions that will be useful throughout the paper.

- The set $\mathbb{R}, \mathbb{R}^{2}$ are endowed, respectively, with $\mathcal{B}(\mathbb{R}), \mathcal{B}\left(\mathbb{R}^{2}\right)$, their Borel tribes.

- The elements of $\mathbb{R}^{2}$ are treated like line vectors. If $(x, z) \in \mathbb{R}^{2}$ and $\delta>0, B((x, z), \epsilon)=$ $\left\{\left(x^{\prime}, z^{\prime}\right) \in \mathbb{R}^{2}: \sqrt{\left(x-x^{\prime}\right)^{2}+\left(z-z^{\prime}\right)^{2}}<\epsilon\right\}$ (the ball of center $(x, z)$ and radius $\epsilon$ ). The superscript $T$ denotes the transposition. For example, if $(x, z) \in \mathbb{R}^{2}$, then $(x, z)^{T}$ is a column vector.

- The set of probability distributions on a measurable space $(E, \mathcal{F})$ and the set of nonnegative measures on $(E, \mathcal{F})$ are denoted by $\mathcal{P}(E)$ and $\mathcal{M}^{+}(E)$ respectively. We write $\mathcal{C}(E)$ for the set of continuous function on a topological space $E$ and $\mathcal{C}_{b}^{+}(E)$ for the set of bounded, continuous, nonnegative functions on $E$.

- When applied to measures, $\|\ldots\|$ stands for the total variation norm (for $\mu, \nu$ probabilities on a measurable space $\left.(F, \mathcal{F}),\|\mu-\nu\|=\sup _{A \in \mathcal{F}}|\mu(A)-\nu(A)|\right)$.

- For any nonnegative kernel $K$ on a measurable space $E$ and any $\mu \in \mathcal{M}^{+}(E)$, we set

$$
K \mu\left(d v^{\prime}\right)=\int_{E} \mu(d v) K\left(v, d v^{\prime}\right) .
$$

- If we have a sequence of nonnegative kernels $K_{1}, K_{2}, \ldots$ on some measured spaces $E_{1}, E_{2}$, $\ldots$ (meaning that for all $i \geq 1, x \in E_{i-1}, K_{i}(x,$.$) is a nonnegative measure on E_{i}$, then for all $i<j$, we define the kernel

$$
K_{i+1: j}\left(x_{i}, d x_{j}\right)=\int_{x_{i+1} \in E_{i+1}} \cdots \int_{x_{j-1} \in E_{j-1}} K_{i+1}\left(x_{i}, d x_{i+1}\right) K_{i+2}\left(x_{i+1}, d x_{i+2}\right) \ldots K_{j}\left(x_{j-1}, d x_{j}\right) .
$$

- For any measurable space $E$ and any nonzero $\mu \in \mathcal{M}^{+}(E)$, we define the normalized nonnegative measure,

$$
\bar{\mu}=\frac{1}{\mu(E)} \mu .
$$


- For any measurable space $E$ and any nonnegative kernel $K$ defined on $E$, we define the normalized nonnegative nonlinear operator $\bar{K}$ on $\mathcal{M}^{+}(E)$, taking values in $\mathcal{P}(E)$, and defined by

$$
\bar{K}(\mu)=\frac{K \mu}{(K \mu)(E)}=\frac{K \bar{\mu}}{(K \bar{\mu})(E)}=\bar{K}(\bar{\mu}),
$$

for any $\mu \in \mathcal{M}^{+}(E)$ such that $K \mu(E) \neq 0$, and defined by $\bar{K}(\mu)=0$ otherwise.

- A kernel $K$ from a measurable space $E_{1}$ into another measurable space $E_{2}$ is said to be $\epsilon$-mixing $(\epsilon \in(0,1))$ if there exists $\lambda$ in $\mathcal{M}^{+}\left(E_{2}\right)$ and $\epsilon_{1}, \epsilon_{2}>0$ such that, for all $x_{1}$ in $E_{1}$,

$$
\epsilon_{1} \lambda(.) \leq K\left(x_{1}, .\right) \leq \frac{1}{\epsilon_{2}} \lambda(.) \text { with } \epsilon_{1} \epsilon_{2}=\epsilon^{2} .
$$

This property implies that, for all $A, \mu, \bar{K}(\mu)(A) \geq \epsilon^{2} \bar{\lambda}(A)$. If $\bar{K}$ is Markov, this last inequality implies that $\bar{K}$ is $\left(1-\epsilon^{2}\right)$-contracting in total variation (see [DG01] p. 161-162 for more details):

$$
\forall \mu, \nu \in \mathcal{P}(E),\|\bar{K}(\mu)-\bar{K}(\nu)\| \leq\left(1-\epsilon^{2}\right)\|\mu-\nu\| .
$$

- For any measurable space $E$ and any $\psi: E \rightarrow \mathbb{R}^{+}$(measurable) and $\mu \in \mathcal{M}^{+}(E)$, we set

$$
\langle\mu, \psi\rangle=\int_{E} \psi(x) \mu(d x) .
$$

If in addition, $\langle\mu, \psi\rangle>0$, we set

$$
\psi \bullet \mu(d v)=\frac{1}{\langle\mu, \psi\rangle} \times \psi(v) \mu(d v) .
$$

- For $\mu$ and $\mu^{\prime}$ in $\mathcal{M}^{+}(E)\left((E, \mathcal{F})\right.$ being a measurable space), we say that $\mu$ and $\mu^{\prime}$ are comparable if there exist positive constants $a$ and $b$ such that, for all $A \in \mathcal{F}$,

$$
a \mu^{\prime}(A) \leq \mu(A) \leq b \mu^{\prime}(A) .
$$

We then define the Hilbert metric between $\mu$ and $\mu^{\prime}$ by

$$
h\left(\mu, \mu^{\prime}\right)=\log \left(\frac{\sup _{A \in \mathcal{F}: \mu^{\prime}(A)>0} \frac{\mu(A)}{\mu^{\prime}(A)}}{\inf _{A \in \mathcal{F}: \mu^{\prime}(A)>0} \frac{\mu(A)}{\mu^{\prime}(A)}}\right) .
$$

It is easily seen (see for instance [Oud00], Chapter 2) that, for any nonnegative kernal $K$ and any $A$ in $\mathcal{F}$,

$$
\begin{aligned}
& h\left(K \mu, K \mu^{\prime}\right) \leq h\left(\mu, \mu^{\prime}\right), \\
& h\left(\bar{\mu}, \overline{\mu^{\prime}}\right) \leq h\left(\mu, \mu^{\prime}\right), \\
& \exp \left(-h\left(\mu, \mu^{\prime}\right)\right) \leq \frac{\mu(A)}{\mu^{\prime}(A)} \leq \exp \left(h\left(\mu, \mu^{\prime}\right)\right), \text { if } \mu^{\prime}(A)>0 .
\end{aligned}
$$

In addition, we have the following relation with the total variation norm:

$$
\left\|\bar{\mu}-\overline{\mu^{\prime}}\right\| \leq \frac{2}{\log (3)} h\left(\mu, \mu^{\prime}\right) .
$$

- We set $\widetilde{Q}$ to be the transition of the chain $\left(X_{k \tau}, X_{(k+1) \tau}\right)_{k \geq 0}$.

- We write $\propto$ between two quantities if they are equal up to a multiplicative constant. 
- For $\psi: \mathbb{R}^{2} \rightarrow \mathbb{R}$, we write $\psi(0,$.$) for the function such that, for all x$ in $\mathbb{R}, \psi(0,).(x)=$ $\psi(0, x)$.

- We write $\lambda_{W}$ for the Wiener measure on $\mathcal{C}([0, \tau])$.

We suppose here that the observation $\left(Y_{t}\right)_{t \geq 0}$ is fixed. For $k \in \mathbb{N}^{*}$ and $x, z \in \mathbb{R}$, we define

$$
\psi_{k}(x, z)=\psi\left(Y_{(k-1) \tau: k \tau}, x, z\right)
$$

(the density $\psi$ is defined in Lemma 2.2). For $x_{1} \in \mathbb{R}, x_{2} \in \mathbb{R}$ and $n \in \mathbb{N}^{*}$, we introduce the nonnegative kernel

$$
R_{n}\left(x_{1}, d x_{2}\right)=\psi_{n}\left(x_{1}, x_{2}\right) Q\left(x_{1}, d x_{2}\right) .
$$

Using the above notations, we now have, for all $n \in \mathbb{N}^{*}$, and for all probability law $\pi_{0}^{\prime}$ (with $\left(\pi_{t}^{\prime}\right)_{t \geq 0}$ defined in Equation (1.3))

$$
\pi_{n \tau}=\bar{R}_{n}\left(\pi_{(n-1) \tau}\right), \pi_{n \tau}^{\prime}=\bar{R}_{n}\left(\pi_{(n-1) \tau}^{\prime}\right)
$$

and for $0<m<n$,

$$
\pi_{n \tau}=\bar{R}_{n} \bar{R}_{n-1} \ldots \bar{R}_{m}\left(\pi_{(m-1) \tau}\right), \pi_{n \tau}^{\prime}=\bar{R}_{n} \bar{R}_{n-1} \ldots \bar{R}_{m}\left(\pi_{(m-1) \tau}^{\prime}\right) .
$$

\subsection{Representation of the optimal filter as the law of a Markov chain}

Regardless of the notations of the other sections, we suppose we have a Markov chain $\left(\mathfrak{X}_{n}\right)_{n \geq 0}$ taking values in measured spaces $E_{0}, E_{1}, \ldots$, with nonnegative kernels $\mathfrak{Q}_{1}, \mathfrak{Q}_{2}, \ldots$ (it might be a non-homogeneous Markov chain). Suppose we have potentials $\Psi_{1}: E_{1} \rightarrow \mathbb{R}_{+}, \Psi_{2}: E_{2} \rightarrow \mathbb{R}_{+}, \ldots$ (measurable functions with values in $\mathbb{R}_{+}$) and a law $\eta_{0}$ on $E_{0}$. We are interested in the sequence of probability measures $\left(\eta_{k}\right)_{k \geq 1}$, respectively on $E_{1}, E_{2}, \ldots$, defined by

$$
\forall k \geq 1, \forall \varphi \in \mathcal{C}_{b}^{+}\left(E_{k}\right), \eta_{k}(f)=\frac{\mathbb{E}_{\eta_{0}}\left(\varphi\left(\mathfrak{X}_{k}\right) \prod_{1 \leq i \leq k} \Psi_{i}\left(\mathfrak{X}_{i}\right)\right)}{\mathbb{E}_{\eta_{0}}\left(\prod_{1 \leq i \leq k} \Psi_{i}\left(\mathfrak{X}_{i}\right)\right)}
$$

where $\eta_{0} \in \mathcal{P}\left(E_{0}\right)$ and the index $\eta_{0}$ means we start with $\mathfrak{X}_{0}$ of law $\eta_{0}$. We will say that $\left(\eta_{k}\right)_{k \geq 0}$ is a Feynman-Kac sequence on $\left(E_{k}\right)_{k \geq 0}$ based on the transitions $\left(\mathfrak{Q}_{k}\right)_{k \geq 1}$, the potentials $\left(\Psi_{k}\right)_{k \geq 1}$ and the initial law $\eta_{0}$. Suppose we have another law $\eta_{0}^{\prime}$, we then set

$$
\forall k \geq 1, \forall \varphi \in \mathcal{C}_{b}^{+}\left(E_{k}\right), \eta_{k}^{\prime}(f)=\frac{\mathbb{E}_{\eta_{0}^{\prime}}\left(\varphi\left(\mathfrak{X}_{k}\right) \prod_{1 \leq i \leq k} \Psi_{i}\left(\mathfrak{X}_{i}\right)\right)}{\mathbb{E}_{\eta_{0}^{\prime}}\left(\prod_{1 \leq i \leq k} \Psi_{i}\left(\mathfrak{X}_{i}\right)\right)}
$$

If the functions $\Psi_{k}$ 's are likelihood associated to observations of a Markov chain with transitions $\mathfrak{Q}_{1}, \mathfrak{Q}_{2}, \ldots$ and initial law $\eta_{0}$, then the measures $\eta_{k}$ 's are optimal filters. We fix $n \geq 1$. We would like to express $\eta_{n}$ as the marginal law of some Markov process. We will do so using ideas from [DG01]. We set, for all $k \in\{1, \ldots, n\}$,

$$
\mathfrak{R}_{k}\left(x, d x^{\prime}\right)=\Psi_{k}\left(x^{\prime}\right) \mathfrak{Q}_{k}\left(x, d x^{\prime}\right) .
$$

We suppose that, for all $k, \mathfrak{R}_{k}$ is $\epsilon_{k}$-mixing (notice that $\mathfrak{Q}_{k}$ being $\epsilon_{k}$-mixing implies that $\mathfrak{R}_{k}$ is $\epsilon_{k}$-mixing). By a simple recursion, we have, for all $n$,

$$
\overline{\Re_{1: n}}=\bar{\Re}_{n} \bar{\Re}_{n-1} \ldots \bar{\Re}_{1} .
$$

We set, for all $k \in\{0,1, \ldots, n-1\}$,

$$
\Psi_{n \mid k}(x)=\int_{x_{k+1} \in E_{k+1}} \cdots \int_{x_{n} \in E_{n}} \mathfrak{R}_{k+1}\left(x, d x_{k+1}\right) \prod_{k+2 \leq i \leq n} \mathfrak{R}_{i}\left(x_{i-1}, d x_{i}\right) .
$$


If $k=n$, we set $\Psi_{n \mid n}$ to be constant equal to 1 . For $k \in\{1,2, \ldots, n\}$, we set

$$
\mathfrak{S}_{n \mid k}\left(x, d x^{\prime}\right)=\frac{\Psi_{n \mid k+1}\left(x^{\prime}\right)}{\Psi_{n \mid k}(x)} \mathfrak{R}_{k+1}\left(x, d x^{\prime}\right) .
$$

From [DG01], we get the following result (a simple proof can also be found in [OR05], Proposition $3.1)$.

Proposition 3.1. The operators $\left(\mathfrak{S}_{n \mid k}\right)_{0 \leq k \leq n-1}$ are Markov kernels. For all $k \in\{0, \ldots, n-1\}$, $\mathfrak{S}_{n \mid k}$ is $\epsilon_{k+1}$-mixing. We have

$$
\begin{aligned}
\eta_{n} & =\mathfrak{S}_{n \mid n-1} \mathfrak{S}_{n \mid n-1} \ldots \mathfrak{S}_{n \mid 0}\left(\Psi_{n \mid 0} \bullet \eta_{0}\right), \\
\eta_{n}^{\prime} & =\mathfrak{S}_{n \mid n-1} \mathfrak{S}_{n \mid n-1} \ldots \mathfrak{S}_{n \mid 0}\left(\Psi_{n \mid 0} \bullet \eta_{0}^{\prime}\right),
\end{aligned}
$$

and

$$
\left\|\eta_{n}-\eta_{n}^{\prime}\right\| \leq \prod_{1 \leq k \leq n}\left(1-\epsilon_{k}^{2}\right) \times\left\|\Psi_{n \mid 0} \bullet \eta_{0}-\Psi_{n \mid 0} \bullet \eta_{0}^{\prime}\right\| .
$$

Following the computations of [OR05], p. 434 (or [Oud00], p.66), we have, for all measurable $\Psi: \mathbb{R}^{2} \rightarrow \mathbb{R}^{+}$,

$$
\left\|\Psi \bullet \eta_{0}-\Psi \bullet \eta_{0}^{\prime}\right\| \leq 2 \int_{x \in E_{0}} \frac{\Psi(x)}{\left\langle\eta_{0}, \Psi\right\rangle}\left|\eta_{0}-\eta_{0}^{\prime}\right|(d x) \leq 2 \inf \left(1, \frac{\|\Psi\|_{\infty}}{\left\langle\eta_{0}, \Psi\right\rangle}\left\|\eta_{0}-\eta_{0}^{\prime}\right\|\right) .
$$

For all $x$ in $E_{0}$, as $\Re_{1}$ is $\epsilon_{1}$-mixing,

$$
\begin{aligned}
\frac{\Psi_{n \mid 0}(x)}{\left\langle\eta_{0}, \Psi_{n \mid 0}\right\rangle} & =\frac{\int_{z \in E_{1}} \mathfrak{R}_{2: n}\left(z, E_{n}\right) \mathfrak{R}_{1}(x, d z)}{\int_{y \in E_{0}} \int_{z \in E_{1}} \mathfrak{R}_{2: n}\left(z, E_{n}\right) \mathfrak{R}_{1}(y, d z) \eta_{0}(d y)} \\
\text { (for some } \left.\epsilon_{1}^{\prime}, \epsilon_{1}^{\prime \prime}, \lambda_{1} \text { with } \epsilon_{1}^{\prime} \epsilon_{1}^{\prime \prime}=\epsilon_{1}^{2}\right) & \leq \frac{\int_{z \in E_{1}} \mathfrak{R}_{2: n}\left(z, E_{n}\right) \frac{1}{\epsilon_{1}^{\prime}} \lambda_{1}(d z)}{\int_{z \in E_{1}} \mathfrak{R}_{2: n}\left(z, E_{n}\right) \epsilon_{1}^{\prime \prime} \lambda_{1}(d z)}=\frac{1}{\epsilon_{1}^{2}} .
\end{aligned}
$$

\section{Truncated filter}

We introduce in this section a filter built with truncated likelihoods. We will call it truncated filter or robust filter, the use of the adjective "robust" refers to the fact that it has stability properties (it appears in the proof of Proposition 1.5 below).

\subsection{Integrals of the potential}

We are look here at $\widehat{\psi}\left(y_{0: \tau}, x, z\right)$ for some $x, z$ in $\mathbb{R}$ and a fixed observation $y_{0: \tau}$ between 0 and $\tau$. All what will be said is also true for observations between $k \tau$ and $(k+1) \tau$ (for any $k$ in $\mathbb{N}$ ). From Equations (7.21), (7.23), (7.24), we see that $A_{1}^{y_{0: \tau}}, B_{1}^{y_{0: \tau}}$ are polynomials of degree 1 in $\lambda_{1}, \ldots$, $\lambda_{3}$ and that $C_{1}$ does not depend on $y_{0: \tau}$. We fix $x$ and $z$ in $\mathbb{R}$. Recall that, by Equation (2.8), Lemmas 7.1 and 7.2, $\lambda_{1}, \lambda_{2}, \lambda_{3}$ are functions of $y_{0: \tau}$ and that they can be expressed as integrals of deterministic functions against $d y_{\tau s}$ (this requires some integrations by parts, see Lemma 7.4). Under the law $\widetilde{\mathbb{P}}$ (defined in Equation (7.1)), conditioned to $X_{0}=x, X_{\tau}=z$, we can write

$$
X_{t}=\left(1-\frac{s}{\tau}\right) x+\frac{s}{\tau} z+\widetilde{B}_{s}-\frac{s}{\tau} \widetilde{B}_{\tau}
$$

where $\left(\widetilde{B}_{s}\right)_{s \geq 0}$ is a Brownian motion, independent of $W$. And we can write, using integration by parts and Equation (7.24) (see Lemma 7.5),

$$
A_{1}^{Y_{0: \tau}}=\int_{0}^{\tau}\left(f_{1}(s) d W_{s}+f_{2}(s) d X_{s}\right),
$$


for some deterministic functions $f_{1}, f_{2}$ (and the same goes for $B_{1}^{Y_{0: \tau}}$, see Equation (7.23)).

We set

$$
p_{1,1}=\left(1-\frac{C_{1}^{2}}{4 A_{2} B_{2}}\right)_{+}^{1 / 2}, p_{2,1}=-\frac{C_{1}}{2 B_{2}}, p_{2,2}=1 .
$$

Fact 4.1. We now fix a parameter $\iota \in(1 / 2,1)$. From now on, we suppose that the parameter $\tau$ is chosen such that

$$
\theta \geq 1, A_{2} \geq \frac{h}{4}, B_{2} \geq \frac{h}{4}, C_{1} \leq \frac{h}{8}, \frac{1}{1+p_{2,1}}-\left(2+\frac{4}{h}\right) B_{2} p_{2,1} \theta^{1-\iota}>0, p_{1,1}>\frac{1}{2},\left|p_{2,1}\right| \leq \frac{1}{2} .
$$

This is possible because of Lemma 2.4 and because this Lemma implies: $p_{2,1}=O\left(\theta^{-1}\right)$, $p_{1,1} \underset{\theta \rightarrow+\infty}{\longrightarrow} 1$.

Let us set

$$
\kappa=\left[\begin{array}{cc}
A_{2} & -\frac{C_{1}}{2} \\
-\frac{C_{1}}{2} & B_{2}
\end{array}\right]
$$

If we take

$$
P=\left[\begin{array}{cc}
p_{1,1} & 0 \\
p_{2,1} & p_{2,2}
\end{array}\right]=\left[\begin{array}{cc}
\left(1-\frac{C_{1}^{2}}{4 A_{2} B_{2}}\right)^{1 / 2} & 0 \\
-\frac{C_{1}}{2 B_{2}} & 1
\end{array}\right],
$$

then

$$
\kappa=P^{T}\left[\begin{array}{cc}
A_{2} & 0 \\
0 & B_{2}
\end{array}\right] P \text {. }
$$

We have

$$
P^{-1}=\left[\begin{array}{cc}
\frac{1}{p_{1,1}} & 0 \\
-\frac{p_{2,1}}{p_{1,1}} & 1
\end{array}\right] \text {. }
$$

First, we have to rule out the case where $A_{1}^{y_{0: \tau}}$ and $B_{1}^{y_{0: \tau}}$ are colinear.

Lemma 4.2. The quantities $A_{1}^{y_{0: \tau}}$ and $B_{1}^{y_{0: \tau}}$ are not colinear (as functions of $y_{0: \tau}$ ).

Proof. Suppose there exists $\lambda \in \mathbb{R}$ such that $B_{1}^{y_{0: \tau}}=\lambda A_{1}^{y_{0}: \tau}$ for $\lambda_{W}$-almost all $y_{0: \tau}$. We have, for all $\varphi$ in $\mathcal{C}_{b}^{+}(\mathbb{R})$, using Lemma 2.2 (remember Equations (2.3), (2.4), (2.9), (2.12), (2.15))

$$
\begin{aligned}
\int_{\mathcal{C}([0 ; \tau])} \varphi\left(A_{1}^{y_{0: \tau}}\right) \psi\left(y_{0: \tau}, x, z\right) \lambda_{W}\left(d y_{0: \tau}\right) & \\
\leq & e^{2 M|x-z|+\tau\left(M+\frac{M^{2}}{2}\right)} \int_{\mathcal{C}([0 ; \tau])} \varphi\left(A_{1}^{y_{0: \tau}}\right) \widehat{\psi}\left(y_{0: \tau}, x, z\right) \lambda_{W}\left(d y_{0: \tau}\right) \\
= & \left.\sigma_{1} \sigma_{2} e^{2 M|x-z|+\tau\left(M+\frac{M^{2}}{2}\right.}\right) \int_{\mathbb{R}} \varphi(t) \exp \left(-A_{2} x^{2}-B_{2} z^{2}+C_{1} x z+t x+\lambda t z\right) \Psi^{\prime}(t) d t
\end{aligned}
$$

where

$$
\Psi^{\prime}(t)=\mathbb{E}^{\mathbb{P}}\left(\exp \left(C_{0}^{W_{0: \tau}}\right) \mid A_{1}^{W_{0: \tau}}=t\right) .
$$

We know the last integral is finite (because $\int_{\mathcal{C}([0,1])} \psi\left(y_{0: \tau}, x, z\right)=1$ and because of Lemma 2.2). We introduce $\Psi_{1}^{\prime}$ such that

$$
\Psi^{\prime}(t)=\exp \left(-\frac{1}{4}(t, \lambda t) \kappa^{-1}(t, \lambda t)^{T}\right) \Psi_{1}^{\prime}(t)
$$

and

$$
\forall\left(t_{1}, t_{2}\right) \in \mathbb{R}^{2}, \mathcal{Q}\left(t_{1}, t_{2}\right)=\exp \left(-\frac{1}{4}\left(t_{1}, t_{2}\right) \kappa^{-1}\left(t_{1}, t_{2}\right)^{T}\right)
$$


We have, for all $t,($ remember that $\widetilde{\mathbb{P}}$ is defined in Equation (7.1))

$$
e^{-M\left|X_{0}-X_{t}\right|-\frac{M t}{2}-\frac{M^{2} t}{2}} \leq\left.\frac{d \mathbb{P}}{d \widetilde{\mathbb{P}}}\right|_{\mathcal{F}_{t}} \leq e^{M\left|X_{t}-X_{0}\right|+\frac{M t}{2}}
$$

(this can be deduced from the computations in the proof of Lemma 2.1). Thus, we have, for all $\varphi$ (the first equality being a consequence of Lemma 2.2)

$$
\begin{aligned}
\int_{\mathcal{C}([0 ; 1])} \varphi\left(A_{1}^{y_{0: \tau}}\right) \psi\left(y_{0: \tau}, x, z\right) \lambda_{W}\left(d y_{0: \tau}\right) & =\mathbb{E}^{\mathbb{P}}\left(\varphi\left(A_{1}^{Y_{0: \tau}}\right) \mid X_{0}=x, X_{\tau}=z\right) \\
& =\frac{\mathbb{E}^{\widetilde{\mathbb{P}}}\left(\left.\varphi\left(A_{1}^{Y_{0: \tau}}\right) \frac{d \mathbb{P}}{d \widetilde{\mathbb{P}}}\right|_{\mathcal{F}_{\tau}} \mid X_{0}=x, X_{\tau}=z\right)}{\mathbb{E}^{\widetilde{\mathbb{P}}}\left(\left.\frac{d \mathbb{P}}{d \mathbb{P}}\right|_{\mathcal{F}_{\tau}} \mid X_{0}=x, X_{\tau}=z\right)} \\
\text { ( by Equation }(4.2)) & \geq e^{-2 M|x-z|-\tau\left(M+\frac{M^{2}}{2}\right)} \int_{\mathbb{R}} \varphi(t) \mathcal{Q}_{x, z}^{\prime \prime}(t) d t
\end{aligned}
$$

for some Gaussian density $\mathcal{Q}_{x, z}^{\prime \prime}$ (the density of $A_{1}^{Y_{0: \tau}}$ knowing $X_{0}=x, X_{\tau}=z$, under $\widetilde{\mathbb{P}}$ ).

From Equations (4.8), (4.9), we deduce that, for $(x, z)$ fixed, we have for almost all $t$,

$$
e^{-2 M|x-z|-\tau\left(M+\frac{M^{2}}{2}\right)} \mathcal{Q}_{x, z}^{\prime \prime}(t) \leq e^{2 M|x-z|+\tau\left(M+\frac{M^{2}}{2}\right)} \sigma_{1} \sigma_{2} \mathcal{Q}((t, \lambda t)-2(x, z) \kappa) \Psi_{1}^{\prime}(t) .
$$

In the same way, for $(x, z)$ fixed, we have for almost all $t$,

$$
e^{2 M|x-z|+\tau\left(M+\frac{M^{2}}{2}\right)} \mathcal{Q}_{x, z}^{\prime \prime}(t) \geq e^{-2 M|x-z|-\tau\left(M+\frac{M^{2}}{2}\right)} \sigma_{1} \sigma_{2} \mathcal{Q}((t, \lambda t)-2(x, z) \kappa) \Psi_{1}^{\prime}(t) .
$$

Looking at Equations (4.1), (4.2), we can say that the density $\mathcal{Q}_{x, z}^{\prime \prime}(t)$ is of the form

$$
\mathcal{Q}_{x, z}^{\prime \prime}(t)=\frac{1}{\sqrt{2 \pi \sigma_{0}^{2}}} \exp \left(-\frac{1}{2 \sigma_{0}^{2}}\left(t-\left(a_{0} x+b_{0} z\right)\right)^{2}\right),
$$

with $\sigma_{0}, a_{0}, b_{0}$ independent of $(x, z)$. So, looking at the above inequalities in $(x, z)=(0,0)$, we see there exists $\epsilon>0$ and a constant $C_{\epsilon}$, such that, for almost all $t$ in $(-\epsilon, \epsilon)$,

$$
\left(C_{\epsilon} \sigma_{1} \sigma_{2}\right)^{-1} e^{-\tau\left(2 M+M^{2}\right)} \leq \Psi_{1}^{\prime}(t) \leq C_{\epsilon}\left(\sigma_{1} \sigma_{2}\right)^{-1} e^{\tau\left(2 M+M^{2}\right)} .
$$

For any $t$, the quantities $\log \left(\mathcal{Q}_{x, z}^{\prime \prime}(t)\right), \log (\mathcal{Q}((t, \lambda t)-2(x, z) \kappa))$ are polynomials in $x, z$, of degree less than 2. Using the above remarks and studying adequate sequences $\left(x_{n}, z_{n}\right)_{n \geq 0}$ (for example, with $x_{n} \underset{n \rightarrow+\infty}{\longrightarrow}+\infty, z_{n}$ remaining in a neighborhood of 0 ), one can show that the coefficients in $x^{2}, z^{2}$ and $x z$ in these two polynomials the same. We then have

$$
\frac{a_{0}^{2}}{2 \sigma_{0}^{2}}=A_{2}, \frac{b_{0}^{2}}{2 \sigma_{0}^{2}}=B_{2}, \frac{a_{0} b_{0}}{\sigma_{0}^{2}}=C_{1} .
$$

By Equation (4.4), we have

$$
\frac{a_{0} b_{0}}{\sigma_{0}^{2}}=2 \sqrt{A_{2} B_{2}} \geq \frac{h}{2}>\frac{h}{8}
$$

and $C_{1} \leq h / 8$, which is not possible, hence the result.

We can now write for any test function $\varphi$ in $\mathcal{C}_{b}^{+}([0, \tau])$ (remember Equation $(2.16)$ )

$$
\int_{\mathcal{C}([0, \tau])} \varphi\left(A_{1}^{y_{0: \tau}}, B_{1}^{y_{0: \tau}}\right) \widehat{\psi}\left(y_{0: \tau}, x, z\right) \lambda_{W}\left(d y_{0: \tau}\right)=
$$




$$
\sigma_{1} \sigma_{2} \int_{\mathbb{R}^{k}} \varphi\left(t_{1}, t_{2}\right) \exp \left[-A_{2} x^{2}-B_{2} z^{2}+C_{1} x z+t_{1} x+t_{2} z\right] \times \Psi\left(t_{1}, t_{2}\right) d t_{1} d t_{2}
$$

where

$$
\Psi\left(t_{1}, t_{1}\right)=\mathbb{E}^{\mathbb{P}}\left(\exp \left(C_{0}^{W_{0: \tau}}\right) \mid A_{1}^{W_{0: \tau}}=t_{1}, B_{1}^{W_{0: \tau}}=t_{2}\right) .
$$

We know the integral is finite (because $\int_{\mathcal{C}([0,1])} \psi\left(y_{0: \tau}, x, z\right) \lambda_{W}\left(d y_{0: \tau}\right)=1$ and because of Lemma 2.2 ). Let us define $\Psi_{1}$ by the formula

$$
\Psi\left(t_{1}, t_{2}\right)=\exp \left(-\frac{1}{4}\left(t_{1}, t_{2}\right) \kappa^{-1}\left(t_{1}, t_{2}\right)^{T}\right) \Psi_{1}\left(t_{1}, t_{2}\right) .
$$

The next result tells us that, somehow, $\log \left(\Psi_{1}\left(t_{1}, t_{2}\right)\right)$ is negligible before $t_{1}^{2}+t_{2}^{2}$ (when $\left(t_{1}, t_{2}\right) \rightarrow$ $+\infty)$.

Lemma 4.3. There exists a constant $C_{1}^{\prime}(h, \tau)$ (continuous in $(h, \tau)$ ) and $\epsilon>0$ such that for all $(x, z)$ and for almost all $\left(t_{1}, t_{2}\right)$ in $B(2(x, z) \kappa, \epsilon)$,

$$
\begin{aligned}
\frac{1}{C_{1}^{\prime}(h, \tau)} \exp \left(-4 M|x-z|-\tau\left(2 M+M^{2}\right)\right) & \leq \Psi_{1}\left(t_{1}, t_{2}\right) \\
\leq & C_{1}^{\prime}(h, \tau) \exp \left(4 M|x-z|+\tau\left(2 M+M^{2}\right)\right) .
\end{aligned}
$$

Proof. We fix $(x, z)$ in $\mathbb{R}^{2}$. Similarly as in Equation (4.8), we get, using Lemma 2.2, for all $\varphi \in \mathcal{C}_{b}^{+}\left(\mathbb{R}^{2}\right)$,

$$
\begin{gathered}
\int_{\mathcal{C}([0, \tau])} \varphi\left(A_{1}^{y_{0: \tau}}, B_{1}^{y_{0: \tau}}\right) \psi\left(y_{0: \tau}, x, z\right) \lambda_{W}\left(d y_{0: \tau}\right) \\
\leq \int_{\mathcal{C}([0, \tau])} \varphi\left(A_{1}^{y_{0: \tau}}, B_{1}^{y_{0: \tau}}\right) e^{2 M|x-z|+\tau\left(M+\frac{M^{2}}{2}\right)} \widehat{\psi}\left(y_{0: \tau}, x, z\right) \lambda_{W}\left(d y_{0: \tau}\right) \\
=\sigma_{1} \sigma_{2} e^{2 M|x-z|+\tau\left(M+\frac{M^{2}}{2}\right)} \int_{\mathbb{R}^{2}} \varphi\left(t_{1}, t_{2}\right) e^{-A_{2} x^{2}-B_{2} z^{2}+C_{1} x z+t_{1} x+t_{2} z} \Psi\left(t_{1}, t_{2}\right) d t_{1} d t_{2} \\
=\sigma_{1} \sigma_{2} e^{2 M|x-z|+\tau\left(M+\frac{M^{2}}{2}\right)} \int_{\mathbb{R}^{2}} \varphi\left(t_{1}, t_{2}\right) \exp \left(-\frac{1}{4}\left(\left(t_{1}, t_{2}\right)-2(x, z) \kappa\right) \kappa^{-1}\left(\left(t_{1}, t_{2}\right)^{T}-2 \kappa(x, z)^{T}\right)\right) \\
\times \Psi_{1}\left(t_{1}, t_{2}\right) d t_{1} d t_{2} .
\end{gathered}
$$

Similarly as in Equation (4.9), we get, for all $\varphi$,

$$
\begin{aligned}
& \int_{\mathcal{C}([0, \tau])} \varphi\left(A_{1}^{y_{0: \tau}}, B_{1}^{y_{0: \tau}}\right) \psi\left(y_{0: \tau}, x, z\right) \lambda_{W}\left(d y_{0: \tau}\right) \leq e^{2 M|z-x|+\tau\left(M+\frac{M^{2}}{2}\right)} \int_{\mathbb{R}^{2}} \varphi\left(t_{1}, t_{2}\right) \mathcal{Q}_{x, z}^{\prime}\left(t_{1}, t_{2}\right) d t_{1} d t_{2}, \\
& e^{-2 M|z-x|-\tau\left(M+\frac{M^{2}}{2}\right)} \int_{\mathbb{R}^{2}} \varphi\left(t_{1}, t_{2}\right) \mathcal{Q}_{x, z}^{\prime}\left(t_{1}, t_{2}\right) d t_{1} d t_{2} \leq \int_{\mathcal{C}([0, \tau])} \varphi\left(A_{1}^{y_{0: \tau}}, B_{1}^{y_{0: \tau}}\right) \psi\left(y_{0: \tau}, x, z\right) \lambda_{W}\left(d y_{0: \tau}\right),
\end{aligned}
$$

for some Gaussian density $\mathcal{Q}_{x, z}^{\prime}$ with covariance matrix which does not depend on $x, z$ (see Equation (4.2)). This is the density of $\left(A_{1}^{Y_{0: \tau}}, B_{1}^{Y_{0: \tau}}\right)$ knowing $X_{0}=x$ and $X_{\tau}=z$, under $\widetilde{\mathbb{P}}$. We then have, a.s. in $\left(t_{1}, t_{2}\right)$ (for the Lebesgue measure),

$$
\mathcal{Q}_{x, z}^{\prime}\left(t_{1}, t_{2}\right) \leq \sigma_{1} \sigma_{2} e^{4 M|x-z|+\tau\left(2 M+M^{2}\right)} \mathcal{Q}\left(\left(t_{1}, t_{2}\right)-2(x, z) \kappa\right) \Psi_{1}\left(t_{1}, t_{2}\right) .
$$

Using the lower bound in the inequality in Lemma 2.2, we get in the same way, a.s. in $\left(t_{1}, t_{2}\right)$,

$$
\mathcal{Q}_{x, z}^{\prime}\left(t_{1}, t_{2}\right) \geq \sigma_{1} \sigma_{2} e^{-4 M|x-z|-\tau\left(2 M+M^{2}\right)} \mathcal{Q}\left(\left(t_{1}, t_{2}\right)-2(x, z) \kappa\right) \Psi_{1}\left(t_{1}, t_{2}\right) .
$$

We deduce from Equation (4.17), that there exists $\epsilon_{1}>0$ such that, for all $(x, z)$ and for almost all $\left(t_{1}, t_{2}\right)$ in $B\left(2(x, z) \kappa, \epsilon_{1}\right)$

$$
\Psi_{1}\left(t_{1}, t_{2}\right) \leq C_{1}^{\prime}(\tau, h) e^{4 M|x-z|+\tau\left(2 M+M^{2}\right)},
$$


for some function $C_{1}^{\prime}(\tau, h)$ of the parameters $\tau, h$ (continuous in $(h, \tau)$ ). Looking at Equations (4.1), (4.2), one can also see that $\mathcal{Q}_{x, z}^{\prime}$ reaches its maximum at $(x, z) \kappa^{\prime}$, where $\kappa^{\prime}$ is fixed in $\mathcal{M}_{2,2}(\mathbb{R})$ (the set of $2 \times 2$ matrices with coefficients in $\mathbb{R}$ ). From Equation (4.16), we get that there exists $\epsilon_{2}>0$ such that, for all $x, z$ and for almost all $\left(t_{1}, t_{2}\right)$ in $B\left((x, z) \kappa^{\prime}, \epsilon_{2}^{\prime}\right)$,

$$
\begin{aligned}
\mathcal{Q}\left(\left(t_{1}, t_{2}\right)-2(x, z) \kappa\right) \geq \frac{1}{2} \mathcal{Q}_{x, z}^{\prime}\left((x, z) \kappa^{\prime}\right)\left(\sigma_{1} \sigma_{2}\right)^{-1} e^{-4 M|x-z|-\tau\left(4 M+2 M^{2}\right)}\left(C_{1}^{\prime}(h, \tau)\right)^{-1} & \\
& \times \exp \left(-4 M\left|\frac{1}{2}(x, z) \kappa^{\prime} \kappa^{-1}(1,-1)^{T}\right|\right),
\end{aligned}
$$

and so, by continuity,

$$
\begin{aligned}
\mathcal{Q}\left((x, z) \kappa^{\prime}-2(x, z) \kappa\right) \geq \frac{1}{2} \mathcal{Q}_{x, z}^{\prime}\left((x, z) \kappa^{\prime}\right)\left(\sigma_{1} \sigma_{2}\right)^{-1} e^{-4 M|x-z|-\tau\left(4 M+2 M^{2}\right)}\left(C_{1}^{\prime}(h, \tau)\right)^{-1} & \\
& \times \exp \left(-4 M\left|\frac{1}{2}(x, z) \kappa^{\prime} \kappa(1,-1)^{T}\right|\right),
\end{aligned}
$$

If $\kappa^{\prime} \neq 2 \kappa$, we can find a sequence $\left(x_{n}, z_{n}\right)$ such that $x_{n}^{2}+z_{n}^{2} \underset{n \rightarrow+\infty}{\longrightarrow}+\infty$ and

$$
\log \left(\mathcal{Q}\left(\left(x_{n}, z_{n}\right) \kappa^{\prime}-2\left(x_{n}, z_{n}\right) \kappa\right)\right) \preceq-\left(x_{n}^{2}+z_{n}^{2}\right),
$$

whereas

$$
\begin{array}{r}
\log \left(\mathcal{Q}_{x_{n}, z_{n}}^{\prime}\left(\left(x_{n}, z_{n}\right) \kappa^{\prime}\right)\left(\sigma_{1} \sigma_{2}\right)^{-1} e^{-4 M\left|x_{n}-z_{n}\right|-\tau\left(4 M+2 M^{2}\right)} \exp \left(-4 M\left|\frac{1}{2}(x, z) \kappa^{\prime} \kappa^{-1}(1,-1)^{T}\right|\right)\right) \\
\succeq-\left|x_{n}\right|-\left|z_{n}\right|,
\end{array}
$$

which is not possible. So $\kappa^{\prime}=2 \kappa$.

So, we get from Equations (4.16), (4.17) that there exists $\epsilon_{3}>0$ such that for all $x, z$, and for almost all $\left(t_{1}, t_{2}\right)$ in $B\left(2(x, z) \kappa, \epsilon_{3}\right)$,

$$
C_{1}^{\prime}(h, \tau) e^{4 M|x-z|+\tau\left(2 M+M^{2}\right)} \geq \Psi_{1}\left(t_{1}, t_{2}\right) \geq \frac{e^{-4 M|x-z|-\tau\left(2 M+M^{2}\right)}}{C_{1}^{\prime}(h, \tau)}
$$

(with, possibly, a new $C_{1}^{\prime}(h, \tau)$ ).

Lemma 4.4. If we have a set $\mathcal{A}=\left\{y_{0: \tau} \in \mathcal{C}([0 ; \tau]):\left(A_{1}^{y_{0}: \tau}, B_{1}^{y_{0: \tau}}\right) \in \mathcal{B}\right\}$ for some measurable subset $\mathcal{B}$ of $\mathbb{R}^{2}$, then

$$
\begin{aligned}
& \int_{\mathcal{A}} \widehat{\psi}\left(y_{0: \tau}, x, z\right) \lambda_{W}\left(d y_{0: \tau}\right) \leq \sigma_{1} \sigma_{2} C_{1}^{\prime}(h, \tau) \\
& \times \int_{\left(t_{2}, t_{2}\right) \in \mathcal{B}} \exp \left\{-\frac{A_{2}^{-1}}{4}\left(\left|\frac{t_{1}}{p_{1,1}}-\frac{p_{2,1} t_{2}}{p_{1,1}}-2 A_{2} p_{1,1} x\right|-4 M \mid \frac{1}{p_{1,1}}+\frac{p_{2,1}}{p_{1,1}}\right)_{+}^{2}\right. \\
&\left.\quad-\frac{B_{2}^{-1}}{4}\left(\left|t_{2}-2 B_{2}\left(p_{2,1} x+z\right)\right|-4 M\right)_{+}^{2}\right\} \\
& \times \exp \left(4 M^{2}(1,-1) \kappa^{-1}(1,-1)^{T}+\tau\left(2 M+M^{2}\right)+4 M|x-z|\right) d t_{1} d t_{2},
\end{aligned}
$$

and

$$
\begin{aligned}
& \int_{\mathcal{A}} \widehat{\psi}\left(y_{0: \tau}, x, z\right) \lambda_{W}\left(d y_{0: \tau}\right) \geq \sigma_{1} \sigma_{2} C_{1}^{\prime}(h, \tau)^{-1} \\
& \times \int_{\left(t_{2}, t_{2}\right) \in \mathcal{B}} \exp \left\{-\frac{A_{2}^{-1}}{4}\left(\left|\frac{t_{1}}{p_{1,1}}-\frac{p_{2,1} t_{2}}{p_{1,1}}-2 A_{2} p_{1,1} x\right|+4 M\left|\frac{1}{p_{1,1}}+\frac{p_{2,1}}{p_{1,1}}\right|\right)^{2}\right. \\
&\left.\quad-\frac{B_{2}^{-1}}{4}\left(\left|t_{2}-2 B_{2}\left(p_{2,1} x+z\right)\right|+4 M\right)^{2}\right\} \\
& \times \exp \left(4 M^{2}(1,-1) \kappa^{-1}(1,-1)^{T}-\tau\left(2 M+M^{2}\right)-4 M|x-z|\right) d t_{1} d t_{2} .
\end{aligned}
$$


Proof. We have (computing as in Equation (4.15))

$$
\begin{aligned}
& \int_{\mathcal{A}} \widehat{\psi}\left(y_{0: \tau}, x, z\right) \lambda_{W}\left(d y_{0: \tau}\right) \\
& \quad=\sigma_{1} \sigma_{2} \int_{\mathcal{B}} \exp \left(-(x, z) \kappa(x, z)^{T}+t_{1} x+t_{2} z\right) \exp \left(-\frac{1}{4}\left(t_{1}, t_{2}\right) \kappa^{-1}\left(t_{1}, t_{2}\right)^{T}\right) \Psi_{1}\left(t_{1}, t_{2}\right) d t_{1} d t_{2}
\end{aligned}
$$

(by Lemma 4.3, for a complete proof see Lemma 7.6)

$$
\begin{aligned}
\leq \sigma_{1} \sigma_{2} \int_{\mathcal{B}} \exp & \left(-\frac{1}{4}\left[\left(t_{1}, t_{2}\right)^{T}-2 \kappa(x, z)^{T}\right]^{T} \kappa^{-1}\left[\left(t_{1}, t_{2}\right)^{T}-2 \kappa(x, z)^{T}\right]\right) \\
& \times C_{1}^{\prime}(h, \tau) \exp \left(2 M \times\left|(1,-1) \kappa^{-1}\left(t_{1}, t_{2}\right)^{T}\right|+\tau\left(2 M+M^{2}\right)\right) d t_{1} d t_{2},
\end{aligned}
$$

and we can bound by below by

$$
\begin{aligned}
\sigma_{1} \sigma_{2} \int_{\mathcal{B}} \exp \left(-\frac{1}{4}\left[\left(t_{1}, t_{2}\right)^{T}-2 \kappa(x, z)^{T}\right]^{T} \kappa^{-1}\left[\left(t_{1}, t_{2}\right)^{T}-2 \kappa(x, z)^{T}\right]\right) \\
\times \frac{1}{C_{1}^{\prime}(h, \tau)} \exp \left(-2 M \times\left|(1,-1) \kappa^{-1}\left(t_{1}, t_{2}\right)^{T}\right|-\tau\left(2 M+M^{2}\right)\right) d t_{1} d t_{2}
\end{aligned}
$$

For $\left(t_{1}, t_{2}\right) \in \mathbb{R}^{2}$, we have, for any $\delta \in\{-1,1\}$

$$
\begin{gathered}
\exp \left(-\frac{1}{4}\left[\left(t_{1}, t_{2}\right)^{T}-2 \kappa(x, z)^{T}\right]^{T} \kappa^{-1}\left[\left(t_{1}, t_{2}\right)^{T}-2 \kappa(x, z)^{T}\right]+2 \delta M \times(1,-1) \kappa^{-1}\left(t_{1}, t_{2}\right)^{T}\right) \\
=\exp \left(-\frac{1}{4}\left[\left(t_{1}, t_{2}\right)^{T}-2 \kappa(x, z)^{T}-4 \delta M(1,-1)^{T}\right]^{T} \kappa^{-1}\left[\left(t_{1}, t_{2}\right)^{T}-2 \kappa(x, z)^{T}-4 \delta M(1,-1)^{T}\right]\right. \\
\left.+4 M^{2}(1,-1) \kappa^{-1}(1,-1)^{T}+4 \delta M(x, z)(1,-1)^{T}\right) \\
=\exp \left(-\frac{1}{4}\left[\left(P^{-1}\right)^{T}\left(t_{1}, t_{2}\right)^{T}-2\left[\begin{array}{cc}
A_{2} & 0 \\
0 & B_{2}
\end{array}\right] P(x, z)^{T}-4 \delta M\left(P^{-1}\right)^{T}(1,-1)^{T}\right]^{T}\right. \\
\left.\times\left[\begin{array}{cc}
A_{2}^{-1} & 0 \\
0 & B_{2}^{-1}
\end{array}\right]\left[\left(P^{-1}\right)^{T}\left(t_{1}, t_{2}\right)^{T}-2\left[\begin{array}{cc}
A_{2} & 0 \\
0 & B_{2}
\end{array}\right] P(x, z)^{T}-4 \delta M\left(P^{-1}\right)^{T}(1,-1)^{T}\right]\right) \\
\times \exp \left(4 M^{2}(1,-1) \kappa^{-1}(1,-1)^{T}+4 \delta M(x, z)(1,-1)^{T}\right) .
\end{gathered}
$$

From there, we get the result.

\subsection{Truncation}

In the following, the parameter $\Delta>0$ is to be understood as a truncation level. For $k \geq 0$ and $\Delta>0$, we set (for all $b$ )

$$
C_{k+1}(\Delta, b)=\left\{z:\left|2 B_{2}\left(1+p_{2,1}\right) z-b\right| \leq \Delta\right\}
$$

(which indeed does not depend on $k$ ),

$$
C_{k+1}(\Delta)=C_{k+1}\left(\Delta, B_{1}^{y_{k \tau:(k+1) \tau}}\right)
$$

and

$$
m_{k+1}(b)=\frac{b}{2 B_{2}\left(1+p_{2,1}\right)},
$$

(which indeed does not depend on $k$ ) and

$$
m_{k+1}=m_{k+1}\left(B_{1}^{Y_{k \tau:(k+1) \tau}}\right) .
$$


We suppose that $m_{0}$ is a point in the support of $\pi_{0}$ (the law of $X_{0}$ ) and we set

$$
C_{0}(\Delta)=\left[m_{0}-\frac{\Delta}{2 B_{2}\left(1+p_{2,1}\right)}, m_{0}+\frac{\Delta}{2 B_{2}\left(1+p_{2,1}\right)}\right] .
$$

Under Hypothesis 2, there exists constants $C_{0}$ and $C_{0}^{\prime}$ such that

$$
\pi_{0}\left(C_{0}(\Delta)^{\complement}\right) \leq C_{0}^{\prime} e^{-C_{0} \Delta^{2}}, \forall \Delta>0 .
$$

From Equation (4.4) and Lemma 2.7, we see that there exists a universal constant $C$ such that

$$
\left|m_{k}-m_{k-1}\right| \leq \begin{cases}C\left(M \tau+\mathcal{V}_{(k-2) \tau, k \tau}+\left(1+\frac{1}{\theta}\right) \mathcal{W}_{(k-2) \tau, k \tau}\right) & \text { if } k \geq 2 \\ \left|m_{0}\right|+C\left(M \tau+\mathcal{V}_{0,2 \tau}+\left(1+\frac{1}{\theta}\right) \mathcal{W}_{0,2 \tau}\right) & \text { if } k=1\end{cases}
$$

We set

$$
d(\Delta)=\frac{\Delta}{1+p_{2,1}}-\left(2+\frac{16}{h}\right) B_{2} p_{2,1} \theta^{1-\iota} \Delta-4 M,
$$

(because of Equation (4.4), we have $d(\Delta) \underset{\Delta \rightarrow+\infty}{\longrightarrow}+\infty)$ and

$$
\begin{gathered}
T(\Delta)=\frac{C \sqrt{\tau} \exp \left(-\frac{1}{2}\left(\frac{\theta^{1-\iota} \Delta}{12 C \sqrt{2 \tau}}\right)^{2}\right)}{\theta^{1-\iota} \Delta} \\
+\left(M \frac{\left(1+p_{2,1}\right)}{p_{1,1}}+\sqrt{A_{2}}\right) C_{1}^{\prime}(h, \tau) \sigma_{1} \sigma_{2} p_{1,1} \frac{B_{2} e^{26 M \tau+\frac{7 \tau M}{2}+40 M^{2} / h}}{d(\Delta)} \exp \left(-\frac{1}{4 B_{2}} d(\Delta)^{2}\right)+e^{-C_{0} \Delta^{2}} .
\end{gathered}
$$

We define, for all $k \geq 1, x$ and $x^{\prime}$ in $\mathbb{R}$ (recall that $\psi_{k}$ is defined in Equation 3.6)

$$
\begin{gathered}
\psi_{k}^{\Delta}\left(x, x^{\prime}\right)=\psi_{k}\left(x, x^{\prime}\right) \mathbb{1}_{C_{k}(\Delta)}\left(x^{\prime}\right), \\
D_{k}=\left|m_{k}-m_{k-1}\right|
\end{gathered}
$$

and for $D \geq 0$,

$$
\begin{gathered}
\xi_{1}(D, \Delta)=\frac{1}{\sqrt{2 \pi \tau}} \exp \left(-\frac{\left(D+\frac{\Delta}{B_{2}\left(1+p_{2,1}\right)}\right)^{2}}{2 \tau}\right) \exp \left(-M\left(D+\frac{\Delta}{B_{2}\left(1+p_{2,1}\right)}\right)-\left(\frac{\tau}{2}+\frac{\tau^{2}}{2}\right) M\right) \\
\xi_{2}(D, \Delta)=\frac{1}{\sqrt{2 \pi \tau}} \exp \left(-\frac{\left(\left(D-\frac{\Delta}{B_{2}\left(1+p_{2,1}\right)}\right)_{+}\right.}{2 \tau}\right) \exp \left(M\left(D+\frac{\Delta}{B_{2}\left(1+p_{2,1}\right)}\right)+\frac{\tau}{2} M\right)
\end{gathered}
$$

and,

$$
R_{k}^{\Delta}\left(x, d x^{\prime}\right)= \begin{cases}\psi_{k}^{\Delta}\left(x, x^{\prime}\right) Q\left(x, d x^{\prime}\right) & \text { if } x \in C_{k-1}(\Delta) \\ \psi_{k}^{\Delta}\left(x, x^{\prime}\right) \xi_{1}\left(D_{k}, \Delta\right) d x^{\prime} & \text { if } x \notin C_{k-1}(\Delta) .\end{cases}
$$

We define $\left(\pi_{n}^{\Delta}\right)_{n \geq 0}$ by the following

$$
\left\{\begin{array}{l}
\pi_{0}^{\Delta}=\pi_{0} \\
\pi_{k \tau}^{\Delta}=\bar{R}_{k}^{\Delta} \bar{R}_{k-1}^{\Delta} \ldots \bar{R}_{1}^{\Delta}\left(\pi_{0}\right) \quad \text { for all } k \geq 1
\end{array}\right.
$$

The next lemma tells us that the measures $\pi_{k}$ are concentrated on the compacts $C_{k}(\Delta)$. 
Lemma 4.5. If

$$
\theta^{1-\iota} \Delta>3\left|m_{0}\right|+3 C M \tau \text { and } d(\Delta)>0,
$$

then we have, for all $k \geq 0$,

$$
\mathbb{E}\left(\pi_{k \tau}\left(C_{k}(\Delta)^{\complement}\right) \preceq T(\Delta) .\right.
$$

Proof. We suppose $k \geq 1$ (the proof is similar for $k=0$ ). For a measure $\mu$ in $\mathcal{M}^{+}(\mathbb{R}$ ), we define

$$
\widetilde{Q} \mu\left(d x, d x^{\prime}\right)=\mu(d x) Q\left(x, d x^{\prime}\right), \forall x, x^{\prime} \in \mathbb{R},
$$

(recall $\widetilde{Q}$ has been defined as a Markov kernel on $\mathbb{R}^{2}$, so the above is an extension of the definition of $\widetilde{Q})$. We have

$$
\begin{aligned}
& \pi_{k}\left(C_{k}(\Delta)^{\complement}\right) \times \mathbb{1}_{\left|m_{k}-m_{k-1}\right| \leq \Delta \theta^{1-\iota}=} \\
& \mathbb{1}_{\left|m_{k}-m_{k-1}\right| \leq \Delta \theta^{1-\iota}} \int_{\left(x, x^{\prime}\right) \in \mathbb{R}^{2}} \frac{\psi_{k}\left(x, x^{\prime}\right)}{\left\langle\widetilde{Q} \pi_{k-1}, \psi_{k}\right\rangle} \mathbb{1}_{C_{k}(\Delta)^{\mathrm{C}}}(x)\left(\mathbb{1}_{C_{k-1}(2 \Delta)}(x)+\mathbb{1}_{C_{k-1}(2 \Delta)^{\mathrm{C}}}(x)\right) \widetilde{Q} \pi_{k-1}\left(d x, d x^{\prime}\right),
\end{aligned}
$$

and (using the same computations as in [LO03], proof of Proposition 5.3, [Oud00], p. 66)

$$
\begin{gathered}
\mathbb{E}\left(\mathbb{1}_{\left|m_{k}-m_{k-1}\right| \leq \Delta \theta^{1-\iota}} \int_{\mathbb{R}^{2}} \frac{\psi_{k}\left(x, x^{\prime}\right)}{\left\langle\widetilde{Q} \pi_{k-1}, \psi_{k}\right\rangle} \mathbb{1}_{C_{k}(\Delta)^{\mathrm{c}}}\left(x^{\prime}\right) \mathbb{1}_{C_{k-1}(2 \Delta)}(x) \widetilde{Q} \pi_{k-1}\left(d x, d x^{\prime}\right) \mid Y_{0:(k-1) \tau}\right) \\
=\int_{y \in \mathcal{C}([0, \tau])} \mathbb{1}_{\left|m_{k}\left(B_{1}^{y}\right)-m_{k-1}\right| \leq \Delta \theta^{1-\iota}} \\
\times\left(\int_{\mathbb{R}^{2}} \frac{\psi\left(y, x, x^{\prime}\right)}{\left\langle\widetilde{Q} \pi_{k-1}, \psi(y, ., .)\right\rangle} \mathbb{1}_{\left.C_{k}\left(\Delta, B_{1}^{y}\right)^{\mathrm{c}}\left(x^{\prime}\right) \mathbb{1}_{C_{k-1}(2 \Delta)}(x) \widetilde{Q} \pi_{k-1}\left(d x, d x^{\prime}\right)\right)}\right. \\
\times\left(\int_{\left(u, u^{\prime}\right) \in \mathbb{R}^{2}} \widetilde{Q} \pi_{k-1}\left(u, d u^{\prime}\right) \psi\left(y, u, u^{\prime}\right)\right) \lambda_{W}(d y)
\end{gathered}
$$

(by Fubini's theorem)

$=\int_{y \in \mathcal{C}([0,1])} \mathbb{1}_{\left|m_{k}\left(B_{1}^{y}\right)-m_{k-1}\right| \leq \Delta \theta^{1-\iota}} \int_{\mathbb{R}^{2}} \psi\left(y, x, x^{\prime}\right) \mathbb{1}_{C_{k}\left(\Delta, B_{1}^{y}\right) \mathrm{c}}\left(x^{\prime}\right) \mathbb{1}_{C_{k-1}(2 \Delta)}(x) \widetilde{Q} \pi_{k-1}\left(d x, d x^{\prime}\right) \lambda_{W}(d y)$ (using Lemma 2.2 and Lemma 4.4)

$$
\begin{gathered}
\leq \sigma_{1} \sigma_{2} C_{1}^{\prime}(h, \tau) \int_{\left(x, x^{\prime}\right) \in \mathbb{R}^{2}} \int_{\left(t_{1}, t_{2}\right) \in \mathbb{R}^{2}:\left|m_{k}\left(t_{2}\right)-m_{k-1}\right| \leq \Delta \theta^{1-\iota}} e^{6 M\left|x-x^{\prime}\right|+\tau\left(3 M+3 M^{2} / 2\right)+4 M^{2}\left|(1,-1) \kappa^{-1}(1,-1)^{T}\right|} \\
\times e^{-\frac{1}{4 A_{2}}\left(\left|\frac{t_{1}}{p_{1,1}}-\frac{p_{2,1} t_{2}}{p_{1,1}}-2 A_{2} p_{1,1} x\right|-4 M\left|\frac{1+p_{2,1}}{p_{1,1}}\right|\right)^{2}} \times e^{-\frac{1}{4 B_{2}}\left(\left|t_{2}-2 B_{2}\left(p_{2,1} x+x^{\prime}\right)\right|-4 M\right)_{+}^{2}} \\
\times \mathbb{1}_{C_{k}\left(\Delta, t_{2}\right)^{\mathrm{c}}}\left(x^{\prime}\right) \mathbb{1}_{C_{k-1}(2 \Delta)}(x) d t_{1} d t_{2} \widetilde{Q} \pi_{k-1}\left(d x, d x^{\prime}\right)
\end{gathered}
$$

By a similar computation, we get

$$
\begin{array}{r}
\mathbb{E}\left(\int_{\mathbb{R}^{2}} \frac{\psi_{k}\left(x, x^{\prime}\right)}{\left\langle\widetilde{Q} \pi_{k-1}, \psi_{k}\right\rangle} \mathbb{1}_{C_{k}(\Delta)^{\mathrm{c}}}(x) \mathbb{1}_{C_{k-1}(2 \Delta)^{\mathrm{c}}}(x) \widetilde{Q} \pi_{k-1}\left(d x, d x^{\prime}\right) \mid Y_{0:(k-1) \tau}\right) \\
=\int_{y \in \mathcal{C}([0,1])} \int_{\mathbb{R}^{2}} \psi\left(y, x, x^{\prime}\right) \mathbb{1}_{C_{k}\left(\Delta, B_{1}^{y}\right)^{\mathrm{C}}}\left(x^{\prime}\right) \mathbb{1}_{C_{k-1}(2 \Delta)^{\mathrm{C}}}(x) \widetilde{Q} \pi_{k-1}\left(d x, d x^{\prime}\right) \lambda_{W}(d y) \\
\quad \leq \int_{\mathbb{R}^{2}} \mathbb{1}_{C_{k-1}(2 \Delta)^{\mathrm{c}}}(x) \widetilde{Q} \pi_{k-1}\left(d x, d x^{\prime}\right) \leq \pi_{k-1}\left(C_{k-1}(2 \Delta)^{\mathrm{C}}\right) .
\end{array}
$$

For all $t_{2}$ such that $\left|m_{k}\left(t_{2}\right)-m_{k-1}\right| \leq \theta^{1-\iota} \Delta$, for $x \in C_{k-1}(2 \Delta), x^{\prime} \in C_{k}\left(\Delta, t_{2}\right)^{\complement}$,

$$
\left|t_{2}-2 B_{2}\left(p_{2,1} x+x^{\prime}\right)\right|
$$




$$
\begin{gathered}
=\left|-2 B_{2} x^{\prime}+\frac{t_{2}}{p_{2,1}+1}+\frac{p_{2,1}}{p_{2,1}+1} t_{2}-2 B_{2} p_{2,1} x\right| \\
\geq \frac{\Delta}{1+p_{2,1}}-2 B_{2} p_{2,1}\left|m_{k}\left(t_{2}\right)-m_{k-1}\right|-2 B_{2} p_{2,1}\left|m_{k-1}-x\right| \\
\quad \quad \text { (using Equation (4.4)) } \geq \frac{\Delta}{1+p_{2,1}}-\left(2+\frac{16}{h}\right) B_{2} p_{2,1} \theta^{1-\iota} \Delta .
\end{gathered}
$$

So

$$
\begin{aligned}
\mathbb{E}\left(\pi_{k}\left(C_{k}(\Delta)^{\complement}\right)\right) \leq \mathbb{P}\left(\left|m_{k}-m_{k-1}\right| \geq \theta^{1-\iota} \Delta\right)+\mathbb{E}\left(\pi_{k-1}\left(C_{k-1}(2 \Delta)^{\complement}\right)\right) \\
+\sigma_{1} \sigma_{2} C_{1}^{\prime}(h, \tau) \int_{\left(x, x^{\prime}\right) \in \mathbb{R}^{2}} \int_{\left(t_{1}, t_{2}\right) \in \mathbb{R}^{2}:\left|t_{2}-2 B_{2}\left(p_{2,1} x+x^{\prime}\right)\right|>\frac{\Delta}{1+p_{2,1}}-\left(2+\frac{4}{h}\right) \Delta \theta^{1-\iota} B_{2} p_{2,1}} \\
e^{6 M\left|x-x^{\prime}\right|+\tau\left(3 M+3 M^{2} / 2\right)+4 M^{2}(1,-1) \kappa^{-1}(1,-1)^{T}} \\
\times e^{-\frac{1}{4 A_{2}}\left(\left|\frac{t_{1}}{p_{1,1}}-\frac{p_{2,1} t_{2}}{p_{1,1}}-2 A_{2} p_{1,1} x\right|-4 M\left|\frac{1+p_{2,1}}{p_{1,1}}\right|\right)_{+}^{2}} \\
\times e^{-\frac{1}{4 B_{2}}\left(\left|t_{2}-2 B_{2}\left(p_{2,1} x+x^{\prime}\right)\right|-4 M\right)^{2}} d t_{1} d t_{2} \widetilde{Q} \pi_{k-1}\left(d x, d x^{\prime}\right) .
\end{aligned}
$$

We have, for all $x \geq 0$,

$$
\begin{aligned}
\mathbb{P}\left(\mathcal{V}_{0,2 \tau} \geq x\right) & =\mathbb{P}\left(\sup _{s \in[0,2 \tau]} V_{s}-\inf _{s \in[0,2 \tau]} V_{s} \geq x\right) \\
& \leq \mathbb{P}\left(\sup _{s \in[0,2 \tau]} V_{s} \geq x / 2\right)+\mathbb{P}\left(-\inf _{s \in[0,2 \tau]} V_{s} \geq x / 2\right) \\
& =4 \mathbb{P}\left(V_{2 \tau} \geq x / 2\right) \\
& =2 \mathbb{P}\left(2\left|V_{2 \tau}\right| \geq x\right) .
\end{aligned}
$$

And so, we can bound (for all $x$ )

$$
\begin{gathered}
\mathbb{P}\left(\mathcal{V}_{(k-2)_{+} \tau, k \tau} \geq x\right) \leq 2 \mathbb{P}\left(2\left|W_{2 \tau}\right| \geq x\right), \\
\mathbb{P}\left(\mathcal{W}_{(k-2)_{+} \tau, k \tau} \geq x\right) \leq 2 \mathbb{P}\left(2\left|W_{2 \tau}\right| \geq x\right) .
\end{gathered}
$$

So (with the constant $C$ defined in Equation (4.21)), using the inequality

$$
\forall z>0, \int_{z}^{+\infty} \frac{\exp \left(-\frac{t^{2}}{2 \sigma^{2}}\right)}{\sqrt{2 \pi \sigma^{2}}} d t \leq \frac{\sigma \exp \left(-\frac{z^{2}}{2 \sigma^{2}}\right)}{z \sqrt{2 \pi}},
$$

and using Equation (4.21), as $\theta^{1-\iota} \Delta>3\left|m_{0}\right|+3 C M \tau$, we get

$$
\begin{aligned}
\mathbb{P}\left(\left|m_{k}-m_{k-1}\right| \geq \theta^{1-\iota} \Delta\right) & \leq 4 \mathbb{P}\left(2 \times\left|W_{2 \tau}\right| \geq \frac{\theta^{1-\iota} \Delta}{6 C}\right) \\
& =4 \mathbb{P}\left(\left|W_{1}\right| \geq \frac{\theta^{1-\iota} \Delta}{12 C \sqrt{2 \tau}}\right) \\
& \leq \frac{96 C \sqrt{\tau}}{\theta^{1-\iota} \Delta \sqrt{\pi}} \exp \left(-\frac{1}{2}\left(\frac{\theta^{1-\iota} \Delta}{12 C \sqrt{2 \tau}}\right)^{2}\right) .
\end{aligned}
$$

For all $x, x^{\prime}$, we have

$$
\begin{aligned}
\int_{\left(t_{1}, t_{2}\right) \in \mathbb{R}^{2}:\left|t_{2}-2 B_{2}\left(p_{2,1} x+x^{\prime}\right)\right|>\frac{\Delta}{1+p_{2,1}}-\left(2+\frac{16}{h}\right) \Delta \theta^{1-\iota} B_{2} p_{2,1}} & e^{-\frac{1}{4 A_{2}}\left(\left|\frac{t_{1}}{p_{1,1}}-\frac{p_{2,1} t_{2}}{p_{1,1}}-2 A_{2} p_{1,1} x\right|-4 M\left|\frac{1+p_{2,1}}{p_{1,1}}\right|\right)_{+}^{2}}
\end{aligned}
$$




$$
\begin{gathered}
\times e^{-\frac{1}{4 B_{2}}\left(\left|t_{2}-2 B_{2}\left(p_{2,1} x+x^{\prime}\right)\right|-4 M\right)_{+}^{2}} d t_{1} d t_{2} \\
\left(\text { change of variables : }\left(\begin{array}{c}
t_{1}^{\prime} \\
t_{2}^{\prime}
\end{array}\right)=\left(P^{-1}\right)^{T}\left(\begin{array}{c}
t_{1} \\
t_{2}
\end{array}\right)\right) \\
=\int_{\left(t_{1}^{\prime}, t_{2}^{\prime}\right) \in \mathbb{R}^{2}:\left|t_{2}^{\prime}-2 B_{2}\left(p_{2,1} x+x^{\prime}\right)\right|>\frac{\Delta}{1+p_{2,1}}-\left(2+\frac{16}{h}\right) \Delta \theta^{1-\iota} B_{2} p_{2,1}} e^{-\frac{1}{4 A_{2}}\left(\left|t_{1}^{\prime}-2 A_{2} p_{1,1} x\right|-4 M\left|\frac{1+p_{2,1}}{p_{1,1}}\right|\right)_{+}^{2}} \\
\times e^{-\frac{1}{4 B_{2}}\left(\left|t_{2}^{\prime}-2 B_{2}\left(p_{2,1} x+x^{\prime}\right)\right|-4 M\right)^{2}}+p_{1,1} d t_{1}^{\prime} d t_{2}^{\prime} \\
(\text { by }(4.36)) \leq\left(8 M \frac{\left|1+p_{2,1}\right|}{p_{1,1}}+2 \sqrt{\pi A_{2}}\right) \\
\times \frac{4 B_{2}}{d(\Delta)} \exp \left(-\frac{1}{4 B_{2}} d(\Delta)^{2}\right) p_{1,1}
\end{gathered}
$$

We have, by Lemma 2.1 and Equation (4.4),

$$
\begin{aligned}
& \int_{\left(x, x^{\prime}\right) \in \mathbb{R}^{2}} e^{6 M\left|x-x^{\prime}\right|+\tau\left(3 M+3 M^{2} / 2\right)+4 M^{2}(1,-1) \kappa^{-1}(1,-1)^{T}} \widetilde{Q} \pi_{k-1}\left(d x, d x^{\prime}\right) \\
& \leq \int_{\left(x, x^{\prime}\right) \in \mathbb{R}^{2}} \frac{1}{\sqrt{2 \pi \tau}} \exp \left(-\frac{\left(x-x^{\prime}\right)^{2}}{2 \tau}+\right. \\
& \left.+7 M\left|x-x^{\prime}\right|+\tau\left(\frac{7}{2} M+\frac{3}{2} M^{2}\right)+40 M^{2}\right) d x^{\prime} \pi_{k-1}(d x) \\
& \leq 2 \exp \left(\frac{49}{2} M^{2} \tau+\tau\left(\frac{7}{2} M+\frac{3}{2} M^{2}\right)+\frac{40 M^{2}}{h}\right)
\end{aligned}
$$

So we have

$$
\begin{aligned}
\mathbb{E}\left(\pi_{k}\left(C_{k}(\Delta)^{\complement}\right)\right) \leq \mathbb{E}\left(\pi_{k-1}\left(C_{k-1}(2 \Delta)^{\complement}\right)\right)+ & \frac{96 C \sqrt{\tau}}{\theta^{1-\iota} \Delta \sqrt{\pi}} \exp \left(-\frac{1}{2}\left(\frac{\theta^{1-\iota} \Delta}{12 C \sqrt{2 \tau}}\right)^{2}\right) \\
+\left(8 M \frac{\left|1+p_{2,1}\right|}{p_{1,1}}+2\right. & \left.\sqrt{\pi A_{2}}\right) C_{1}^{\prime}(h, \tau) \sigma_{1} \sigma_{2} p_{1,1} \frac{8 B_{2}}{d(\Delta)} \\
& \times \exp \left(-\frac{1}{4 B_{2}} d(\Delta)^{2}+26 M^{2} \tau+\frac{7}{2} \tau M+\frac{40 M^{2}}{h}\right) .
\end{aligned}
$$

Then, by recurrence,

$$
\begin{aligned}
& \mathbb{E}\left(\pi_{k}\left(C_{k}(\Delta)^{\complement}\right)\right) \leq \mathbb{E}\left(\pi_{0}\left(C_{0}\left(2^{k} \Delta\right)^{\complement}\right)\right)+\sum_{i=0}^{k-1}\left[\frac{96 C \sqrt{\tau}}{2^{i} \theta^{1-\iota} \Delta \sqrt{\pi}} \exp \left(-\frac{1}{2}\left(\frac{2^{i} \theta^{1-\iota} \Delta}{12 C \sqrt{2 \tau}}\right)^{2}\right)\right. \\
& \left.+\left(8 M \frac{\left|1+p_{2,1}\right|}{p_{1,1}}+2 \sqrt{\pi A_{2}}\right) C_{1}^{\prime}(h, \tau) \sigma_{1} \sigma_{2} p_{1,1} \frac{8 B_{2} e^{26 M^{2} \tau+\frac{7}{2} \tau M+40 M^{2} / h}}{d\left(2^{i} \Delta\right)} \exp \left(-\frac{1}{4 B_{2}} d\left(2^{i} \Delta\right)^{2}\right)\right] .
\end{aligned}
$$

We have

$$
\begin{aligned}
\frac{1}{d(\Delta)}+\frac{1}{d(2 \Delta)}+\frac{1}{d(4 \Delta)}+\ldots \leq & \frac{1}{d(\Delta)}+\frac{1}{d(\Delta)}+\frac{1}{d(\Delta)+2 \Delta\left(\frac{1}{1+p_{2,1}}-\left(2+\frac{16}{h}\right) B_{2} p_{2,1} \theta^{1-\iota}\right)} \\
& +\frac{1}{d(\Delta)+4 \Delta\left(\frac{1}{1+p_{2,1}}-\left(2+\frac{16}{h}\right) B_{2} p_{2,1} \theta^{1-\iota}\right)}+\ldots \\
\leq & \frac{2}{d(\Delta)}+\frac{1}{\Delta\left(\frac{1}{1+p_{2,1}}-\left(2+\frac{16}{h}\right) B_{2} p_{2,1} \theta^{1-\iota}\right)} \leq \frac{3}{d(\Delta)} .
\end{aligned}
$$

So 


$$
\begin{aligned}
\mathbb{E}\left(\pi_{k}\left(C_{k}(\Delta)^{\complement}\right)\right) \leq \mathbb{E}\left(\pi_{0}\left(C_{0}\left(2^{k} \Delta\right)^{\complement}\right)\right)+\frac{192 C \sqrt{\tau}}{\theta^{1-\iota} \Delta \sqrt{\pi}} \exp \left(-\frac{1}{2}\left(\frac{\theta^{1-\iota} \Delta}{12 C \sqrt{2 \tau}}\right)^{2}\right) \\
+\left(8 M \frac{\left|1+p_{2,1}\right|}{p_{1,1}}+2 \sqrt{\pi A_{2}}\right) C_{1}^{\prime}(h, \tau) \sigma_{1} \sigma_{2} p_{1,1} 8 B_{2} e^{26 M^{2} \tau+\frac{7}{2} \tau M+40 M^{2} / h} \\
\times \frac{3}{d(\Delta)} \exp \left(-\frac{1}{4 B_{2}} d(\Delta)^{2}\right) .
\end{aligned}
$$

And we can conclude because of Hypothesis 2 .

Corollary 4.6. We suppose that $\pi_{0}^{\prime} \in \mathcal{P}(\mathbb{R})$ is such that $\pi_{0}$ and $\pi_{0}^{\prime}$ are comparable. We suppose that $\left(\pi_{t}^{\prime}\right)_{t \geq 0}$ is defined by Equation (1.3). Under the assumption of the previous Lemma, we have, for all $k \geq 0$,

$$
\mathbb{E}\left(\pi_{k \tau}^{\prime}\left(C_{k}(\Delta)^{\complement}\right) \preceq T(\Delta) e^{h\left(\pi_{0}, \pi_{0}^{\prime}\right)} .\right.
$$

Proof. By Equations (3.2), (3.3), (3.8), we have, for all $k$,

$$
h\left(\pi_{k \tau}^{\prime}, \pi_{k \tau}\right) \leq h\left(\pi_{0}^{\prime}, \pi_{0}\right) .
$$

So, by Equation (3.4),

$$
\begin{aligned}
\mathbb{E}\left(\pi_{k \tau}^{\prime}\left(C_{k}(\Delta)^{\complement}\right)\right. & \leq \mathbb{E}\left(e^{h\left(\pi_{0}^{\prime}, \pi_{0}\right)} \pi_{k \tau}\left(C_{k}(\Delta)^{\complement}\right)\right) \\
& \preceq T(\Delta) e^{h\left(\pi_{0}, \pi_{0}^{\prime}\right)} .
\end{aligned}
$$

The next result tells us that $\bar{R}_{k}$ and $\bar{R}_{k}^{\Delta}(k \geq 1)$ are close in some sense (recall that $\pi_{k \tau}^{\prime}=$ $\left.\bar{R}_{k}\left(\pi_{(k-1) \tau}^{\prime}\right)\right)$

Proposition 4.7. We suppose that $\Delta$ satisfies the assumption of the previous Lemma (Equation (4.30)). We suppose that $\left(\pi_{n \tau}^{\prime}\right)_{n \geq 0}$ satisfies the assumptions of the above Corollary. For all $k \geq 1$, we have

$$
\mathbb{E}\left(\left\|\pi_{k \tau}^{\prime}-\bar{R}_{k}^{\Delta}\left(\pi_{(k-1) \tau}^{\prime}\right)\right\|\right) \preceq T(\Delta) e^{2 h\left(\pi_{0}, \pi_{0}^{\prime}\right)} .
$$

Proof. We define measures on $\mathbb{R}^{2}$ (remember Equation (4.31)):

$$
\begin{aligned}
& \mu=\widetilde{Q} \pi_{(k-1) \tau}^{\prime} \\
& =\widetilde{Q}\left(\mathbb{1}_{C_{k-1}(\Delta)^{\mathrm{c}}} \pi_{(k-1) \tau}^{\prime}\right)\left(d x, d x^{\prime}\right)+\left(\mathbb{1}_{C_{k}(\Delta)}\left(x^{\prime}\right)+\mathbb{1}_{C_{k}(\Delta)^{\mathrm{c}}}\left(x^{\prime}\right)\right) \widetilde{Q}\left(\mathbb{1}_{C_{k-1}(\Delta)} \pi_{(k-1) \tau}^{\prime}\right)\left(d x, d x^{\prime}\right) \\
& \begin{array}{r}
\mu^{\prime}\left(d x, d x^{\prime}\right)=\mathbb{1}_{C_{k}(\Delta)}\left(x^{\prime}\right) \widetilde{Q}\left(\mathbb{1}_{C_{k-1}(\Delta)} \pi_{(k-1) \tau}^{\prime}\right)\left(d x, d x^{\prime}\right) \\
+\pi_{(k-1) \tau}^{\prime}\left(C_{k-1}(\Delta)^{\complement}\right) \xi_{1}\left(D_{k}, \Delta\right) \mathbb{1}_{C_{k}(\Delta)}\left(x^{\prime}\right) d x d x^{\prime}
\end{array}
\end{aligned}
$$

where (by a slight abuse of notation)

$$
\begin{aligned}
& \widetilde{Q}\left(\mathbb{1}_{C_{k-1}(\Delta)} \pi_{(k-1) \tau}^{\prime}\right)\left(d x, d x^{\prime}\right)=\mathbb{1}_{C_{k-1}(\Delta)}(x) \pi_{(k-1) \tau}^{\prime}(d x) Q\left(x, d x^{\prime}\right), \\
& \widetilde{Q}\left(\mathbb{1}_{C_{k-1}(\Delta)} \mathrm{c} \pi_{(k-1) \tau}^{\prime}\right)\left(d x, d x^{\prime}\right)=\mathbb{1}_{C_{k-1}(\Delta)^{\mathrm{c}}}(x) \pi_{(k-1) \tau}^{\prime}(d x) Q\left(x, d x^{\prime}\right),
\end{aligned}
$$

By the definition of $R^{\Delta}$ (Equation (4.28)) and computing as in [OR05], p. 433 (or as in [Oud00], p.66), we get

$$
\begin{aligned}
\left\|\pi_{k \tau}^{\prime}-\bar{R}_{k}^{\Delta}\left(\pi_{(k-1) \tau}^{\prime}\right)\right\| & =\left\|\psi_{k} \bullet \mu-\psi_{k} \bullet \mu^{\prime}\right\| \\
(\text { using Equation }(3.12)) & \leq 2 \int_{\mathbb{R}^{2}} \frac{\psi_{k}\left(x, x^{\prime}\right)}{\left\langle\widetilde{Q} \pi_{k-1}^{\prime}, \psi_{k}\right\rangle} \times\left[\mathbb{1}_{C_{k}(\Delta)^{\mathrm{c}}}\left(x^{\prime}\right) \widetilde{Q}\left(\mathbb{1}_{C_{k-1}(\Delta)} \pi_{(k-1) \tau}^{\prime}\right)\left(d x, d x^{\prime}\right)\right.
\end{aligned}
$$




$$
\begin{aligned}
& +\widetilde{Q}\left(\mathbb{1}_{C_{k-1}(\Delta)} \pi_{(k-1) \tau}^{\prime}\right)\left(d x, d x^{\prime}\right) \\
& \left.+\pi_{(k-1) \tau}^{\prime}\left(C_{k-1}(\Delta)^{\complement}\right) \xi_{1}\left(D_{k}, \Delta\right) \mathbb{1}_{C_{k}(\Delta)}\left(x^{\prime}\right) d x d x^{\prime}\right] .
\end{aligned}
$$

We have, by Lemma 2.2 ,

$$
\begin{aligned}
& \mathbb{E}\left(\mathbb{1}_{\left[0, \theta^{1-\iota} \Delta\right]}\left(\left|m_{k}-m_{k-1}\right|\right)\right. \\
& \left.\times \int_{\mathbb{R}^{2}} \frac{\psi_{k}\left(x, x^{\prime}\right)}{\left\langle\widetilde{Q} \pi_{k-1}^{\prime}, \psi_{k}\right\rangle} \times \mathbb{1}_{C_{k}(\Delta)^{\mathrm{c}}}\left(x^{\prime}\right) \widetilde{Q}\left(\mathbb{1}_{C_{k-1}(\Delta)} \pi_{(k-1) \tau}^{\prime}\right)\left(d x, d x^{\prime}\right) \mid Y_{0:(k-1) \tau}\right) \\
& =\int_{y_{0: \tau} \in \mathcal{C}([0, \tau])} \mathbb{1}_{\left[0, \theta^{1-\iota} \Delta\right]}\left(\left|m_{k}\left(B_{1}^{y 0: \tau}\right)-m_{k-1}\left(B_{1}^{Y_{(k-2) \tau:(k-1) \tau}}\right)\right|\right) \\
& \times \int_{\mathbb{R}^{2}} \frac{\psi\left(y_{0: \tau}, x, x^{\prime}\right)}{\left\langle\widetilde{Q} \pi_{k-1}^{\prime}, \psi\left(y_{0: \tau}, . .\right)\right\rangle} \mathbb{1}_{C_{k}\left(\Delta, B_{1}^{y_{0: \tau}}\right)^{\mathrm{c}}}\left(x^{\prime}\right) \widetilde{Q}\left(\mathbb{1}_{C_{k-1}(\Delta)} \pi_{(k-1) \tau}^{\prime}\right)\left(d x, d x^{\prime}\right) \\
& \times\left(\int_{\mathbb{R}^{2}} \widetilde{Q} \pi_{(k-1) \tau}\left(d u, d u^{\prime}\right) \psi\left(y_{0: \tau}, u, u^{\prime}\right)\right) \lambda_{W}\left(d y_{0: \tau}\right) \\
& \text { (using Equations (3.4), (4.40)) } \\
& \leq e^{2 h\left(\pi_{0}, \pi_{0}^{\prime}\right)} \int_{y_{0: \tau} \in \mathcal{C}([0, \tau])} \mathbb{1}_{\left[0, \theta^{1-\iota} \Delta\right]}\left(\left|m_{k}\left(B_{1}^{y_{0: \tau}}\right)-m_{k-1}\left(B_{1}^{Y_{(k-2) \tau:(k-1) \tau}}\right)\right|\right) \\
& \times \int_{\mathbb{R}^{2}} \psi\left(y_{0: \tau}, x, x^{\prime}\right) \mathbb{1}_{C_{k}\left(\Delta, B_{1}^{y} y_{0: \tau}\right)^{\mathrm{C}}}\left(x^{\prime}\right) \mathbb{1}_{C_{k-1}(\Delta)}(x) \widetilde{Q} \pi_{k-1}\left(d x, d x^{\prime}\right) \lambda_{W}\left(d y_{0: \tau}\right) \\
& \text { (using (4.32), (4.33), (4.38), (4.39) and the fact that } C_{k-1}(\Delta) \subset C_{k-1}(2 \Delta) \text { ) } \\
& \preceq T(\Delta) e^{2 h\left(\pi_{0}, \pi_{0}^{\prime}\right)} .
\end{aligned}
$$

We have, in the same way,

$$
\begin{aligned}
\mathbb{E}\left(\int_{\mathbb{R}^{2}} \frac{\psi_{k}\left(x, x^{\prime}\right)}{\left\langle\widetilde{Q} \pi_{k-1}^{\prime}, \psi_{k}\right\rangle} \times \widetilde{Q}\left(\mathbb{1}_{C_{k-1}(\Delta)^{\mathrm{c}}} \pi_{(k-1) \tau}^{\prime}\right)\left(d x, d x^{\prime}\right) \mid Y_{0:(k-1) \tau}\right) \\
\leq e^{h\left(\pi_{0}, \pi_{0}^{\prime}\right)} \int_{y_{0: \tau} \in \mathcal{C}([0, \tau])} \int_{\mathbb{R}^{2}} \psi\left(y_{0: \tau}, x, x^{\prime}\right) \mathbb{1}_{C_{k-1}(\Delta)^{c}(x)}\left(x \pi_{(k-1) \tau}^{\prime}\left(d x, d x^{\prime}\right) \lambda_{W}\left(d y_{0: \tau}\right)\right. \\
=e^{h\left(\pi_{0}, \pi_{0}^{\prime}\right)} \pi_{(k-1) \tau}^{\prime}\left(C_{k-1}(\Delta)^{\complement}\right)
\end{aligned}
$$

and

$$
\begin{array}{r}
\mathbb{E}\left(\int_{\mathbb{R}^{2}} \frac{\psi_{k}\left(x, x^{\prime}\right)}{\left\langle\widetilde{Q} \pi_{k-1}^{\prime}, \psi_{k}\right\rangle} \times \pi_{(k-1) \tau}^{\prime}\left(C_{k-1}(\Delta)^{\complement}\right) \xi_{1}\left(D_{k}, \Delta\right) \mathbb{1}_{C_{k}\left(\Delta, B_{1}^{y}\right)}\left(x^{\prime}\right) d x d x^{\prime} \mid Y_{0:(k-1) \tau}\right) \\
\leq e^{h\left(\pi_{0}, \pi_{0}^{\prime}\right)} \int_{y_{0: \tau} \in \mathcal{C}([0, \tau])} \int_{\mathbb{R}^{2}} \psi\left(y_{0: \tau}, x, x^{\prime}\right) \pi_{(k-1) \tau}^{\prime}\left(C_{k-1}(\Delta)^{\complement}\right) \xi_{1}\left(m_{k}\left(B_{1}^{y_{0: \tau}}\right)-m_{k-1}, \Delta\right) \\
\times \mathbb{1}_{C_{k}\left(\Delta, B_{1}^{y}\right)}\left(x^{\prime}\right) d x d x^{\prime} \lambda_{W}\left(d y_{0: \tau}\right) \\
\leq e^{h\left(\pi_{0}, \pi_{0}^{\prime}\right)} \pi_{(k-1) \tau}^{\prime}\left(C_{k-1}(\Delta)^{\complement}\right) .
\end{array}
$$

So, using Equation (4.37) and Corollary 4.6, we get the result.

\section{New formula for the truncated filter}

We have reduced the problem to a discrete-time problem. For all $n, \pi_{n \tau}$ is the marginal of a Feynman-Kac sequence based on the transition $\widetilde{Q}$ and the potentials $\left(\psi_{k}\right)_{k \geq 1}$ (see Equations (3.6), (3.7), (3.8), Section 3.1 for the definition of $\widetilde{Q}$, Section 3.2 for the definition of a Feynman-Kac sequence). As in [OR05], we restrict the state space to the compacts $\left(C_{k}(\Delta)\right)_{k \geq 0}$. If $\widetilde{Q}$ restricted 
to compacts was mixing, then, due to Proposition 3.1 (and to the remark below Equation (3.10)), $\pi_{n \tau}^{\Delta}$ could be viewed as the law at time $n$ of a Markov chain with contracting Markov kernels ; and so Lemma 1.4 would be relatively easy to prove. By construction, $\widetilde{Q}$ restricted to some compacts cannot be mixing. This is an effect of the fact that the observations are continuous in time.

The purpose of this Section is to find another representation of the sequence $\left(\pi_{n \tau}\right)_{n \geq 0}$ as a Feynman-Kac sequence, in such a way that the underlying Markov operators would be mixing, when restricted to compacts. Looking at Equation (3.9), we see that a Feynman-Kac sequence is a result of the deformation of a measure on trajectories (we weight the trajectories with potentials $\left(\psi_{k}\right)_{k \geq 1}$ ). The main idea of the following is to incorporate the deformations delicately (in two steps), in order to retain something of the mixing property of the operator $Q$ (which is mixing when restricted to compacts).

In this Section, we work with a fixed observation $\left(Y_{s}\right)_{s \geq 0}=\left(y_{s}\right)_{s \geq 0}$.

\subsection{Filter based on partial information}

We define, for all $x=\left(x_{1}, x_{2}\right), x^{\prime}=\left(x_{1}^{\prime}, x_{2}^{\prime}\right)$ in $\mathbb{R}^{2}, k$ in $\mathbb{N}^{*}, n$ in $\mathbb{N}^{*}, n \geq k$, (recall that $\psi_{k}^{\Delta}$ is defined in Equation (4.24) and that $\xi_{1}, \xi_{2}$ are defnied in (4.26), (4.27))

$$
\text { for } \begin{array}{r}
k \geq 2, \widetilde{R}_{k}^{\Delta}\left(x, d x^{\prime}\right)= \begin{cases}\mathbb{1}_{C_{k-1}(\Delta)}\left(x_{1}^{\prime}\right) \psi_{k}^{\Delta}\left(x^{\prime}\right) \widetilde{Q}^{2}\left(x, d x^{\prime}\right) & \text { if } x_{2} \in C_{k-2}(\Delta), \\
\mathbb{1}_{C_{k-1}(\Delta)}\left(x_{1}^{\prime}\right) \psi_{k}^{\Delta}\left(x^{\prime}\right) \xi_{1}\left(D_{k-1}, \Delta\right) d x^{\prime} & \text { otherwise },\end{cases} \\
\psi_{2 n \mid 2 k}^{\Delta}(x)= \begin{cases}\widetilde{R}_{2 n}^{\Delta} \widetilde{R}_{2 n-2}^{\Delta} \cdots \widetilde{R}_{2 k+2}^{\Delta}\left(x, \mathbb{R}^{2}\right) & \text { if } k \leq n-1, \\
1 & \text { if } k=n,\end{cases}
\end{array}
$$

(so $\psi_{2 n \mid 2 k}^{\Delta}(x)$ does not depend on $x_{1}$ ),

$$
S_{2 n \mid 2 k}^{\Delta}\left(x, d x^{\prime}\right)= \begin{cases}\frac{\psi_{2 n \mid 2 k+2}^{\Delta}\left(x^{\prime}\right)}{\psi_{2 n \mid 2 k}^{\Delta}(x)} \widetilde{R}_{2 k+2}^{\Delta}\left(x, d x^{\prime}\right) & \text { if } k \leq n-1 \\ d x^{\prime} & \text { if } k=n-1\end{cases}
$$

These notations come from [DG01]. As $Q$ has a density with respect to the Lebesgue measure on $\mathbb{R}$, so has $S_{2 n \mid 2 k}^{\Delta}$ (with respect to the Lebesgue measure on $\mathbb{R}^{2}$ ). We write $\left(x, x^{\prime}\right) \in E^{2} \mapsto S_{2 n \mid 2 k}^{\Delta}\left(x, x^{\prime}\right)$ for this density. We fix $n$ in $\mathbb{N}^{*}$ in the rest of this subsection and in the following subsection. We define $S_{2 n \mid 2 k}^{\Delta,(p)}, \psi_{2 n \mid 2 k}^{\Delta,(p)}$, for $0 \leq k \leq n$, with the same formulas used above to define $S_{2 n \mid 2 k}^{\Delta}, \psi_{2 n \mid 2 k}^{\Delta}$, except we replace $\psi_{2 n}^{\Delta}$ by 1 . For all $D>0$, we set

$$
\epsilon(D, \Delta)=\frac{\xi_{1}(D, \Delta)}{\xi_{2}(D, \Delta)}
$$

and, for all $k$,

$$
\epsilon_{k}=\epsilon\left(D_{k}, \Delta\right)
$$

Lemma 5.1. For $k \leq n-1, S_{2 n \mid 2 k}^{\Delta}$ is a Markov operator and $S_{2 n \mid 2 k}^{\Delta}$ is $\left(1-\epsilon_{2 k+1}^{2}\right)$-contracting for the total variation norm, $S_{2 n \mid 2 k}^{\Delta,(p)}$ is a Markov operator and $S_{2 n \mid 2 k}^{\Delta,(p)}$ is $\left(1-\epsilon_{2 k+1}^{2}\right)$-contracting for the total variation norm

Proof. We write the proof only for the kernels $S_{\ldots}^{\Delta}$, it would be very similar for the kernels $S_{\ldots}^{\Delta,(p)}$. By Proposition 3.1, $S_{2 n \mid 2 k}^{\Delta}$ is a Markov operator. We set, for all $k \geq 1, x_{1}, x_{2}$ in $\mathbb{R}$,

$$
\lambda_{k}\left(d x_{1}, d x_{2}\right)=\mathbb{1}_{C_{k-1}(\Delta)}\left(x_{1}\right) \mathbb{1}_{C_{k}(\Delta)}\left(x_{2}\right) \psi_{k}\left(x_{1}, x_{2}\right) Q\left(x_{1}, x_{2}\right) d x_{1} d x_{2} .
$$

By Lemma 2.1, we have, for all $x_{1}, x_{2}, z_{1}, z_{2}$ in $\mathbb{R}, k \geq 2$ (we use here the second line of Equation $(5.1))$

$$
\xi_{1}\left(D_{k-1}, \Delta\right) \lambda_{k}\left(d z_{1}, d z_{2}\right) \leq \widetilde{R}_{k}^{\Delta}\left(x_{1}, x_{2}, d z_{1}, d z_{2}\right) \leq \xi_{2}\left(D_{k-1}, \Delta\right) \lambda_{k}\left(d z_{1}, d z_{2}\right)
$$


So $\widetilde{R}_{k}^{\Delta}$ is $\sqrt{\epsilon_{k-1}}$-mixing. So, for all $x$ in $\mathbb{R}^{2}$, all $k$ such that $0 \leq k \leq n-1$ (the convention being that, if $\left.k=n-1,\left(\widetilde{R}_{2 n}^{\Delta} \ldots \widetilde{R}_{2 k+4}^{\Delta}\right)(y, d z)=\delta_{y}(d z)\right)$

$$
\begin{aligned}
\psi_{2 n \mid 2 k}^{\Delta}(x) & =\int_{\mathbb{R}^{2} \times \mathbb{R}^{2}}\left(\widetilde{R}_{2 n}^{\Delta} \ldots \widetilde{R}_{2 k+4}^{\Delta}\right)(y, d z) \widetilde{R}_{2 k+2}^{\Delta}(x, d y) \\
& \leq \int_{\mathbb{R}^{2} \times \mathbb{R}^{2}}\left(\widetilde{R}_{2 n}^{\Delta} \ldots \widetilde{R}_{2 k+4}^{\Delta}\right)(y, d z) \xi_{2}\left(D_{2 k+1}, \Delta\right) \lambda_{2 k+2}(d y)
\end{aligned}
$$

and, for $x^{\prime}$ in $\mathbb{R}^{2}$,

$$
\widetilde{R}_{2 k+2}^{\Delta}\left(x, d x^{\prime}\right) \geq \xi_{1}\left(D_{2 k+1}, \Delta\right) \lambda_{2 k+2}\left(d x^{\prime}\right)
$$

SO

$$
S_{2 n \mid 2 k}^{\Delta}\left(x, d x^{\prime}\right) \geq \frac{\xi_{1}\left(D_{2 k+1}, \Delta\right)}{\xi_{2}\left(D_{2 k+1}, \Delta\right)} \times \frac{\widetilde{R}_{2 k+4: 2 n}^{\Delta}\left(x^{\prime}, \mathbb{R}^{2}\right) \lambda_{2 k+2}\left(d x^{\prime}\right)}{\int_{\mathbb{R}^{2}} \widetilde{R}_{2 k+4: 2 n}^{\Delta}(y, \mathbb{R}) \lambda_{2 k+2}(d y)} .
$$

In the same way as above, we can also obtain

$$
S_{2 n \mid 2 k}^{\Delta}\left(x, d x^{\prime}\right) \leq \frac{\xi_{2}\left(D_{2 k+1}, \Delta\right)}{\xi_{1}\left(D_{2 k+1}, \Delta\right)} \times \frac{\widetilde{R}_{2 k+4: 2 n}\left(x^{\prime}, \mathbb{R}^{2}\right) \lambda_{2 k+2}\left(d x^{\prime}\right)}{\int_{\mathbb{R}^{2}} \widetilde{R}_{2 k+4: 2 n}\left(y, \mathbb{R}^{2}\right) \lambda_{2 k+2}(d y)} .
$$

This implies that $S_{2 n \mid 2 k}^{\Delta}$ is $\left(1-\epsilon_{2 k+1}^{2}\right)$-contracting for the total variation norm (see Subsection 3.1). One can also use Proposition 3.1 to prove this result. We did it this way because we will re-use Equations (5.5), (5.6).

We set $Z_{0}$ to be of the form $Z_{0}=\left(0, Z_{0}^{(2)}\right)$, with $Z_{0}^{(2)}$ a random variable. We set $\left(Z_{2 k}\right)_{0 \leq k \leq n}$ to be a non-homogeneous Markov chain with kernels $S_{2 n \mid 0}^{\Delta}, S_{2 n \mid 2}^{\Delta}, \ldots, S_{2 n \mid 2 n-2}^{\Delta}$ (for $k$ in $\{1,2, \ldots, n\}$, the law of $Z_{2 k}$ knowing $Z_{2 k-2}$ is $\left.S_{2 n \mid 2 k-2}^{\Delta}\left(Z_{2 k-2},.\right)\right)$. For $Z_{2 k}$ being a element of this chain, we denote by $Z_{2 k}^{(1)}$ and $Z_{2 k}^{(2)}$ its first and second component respectively. Recalling Proposition 3.1 (or Proposition 3.1, p. 428 in [OR05], or similar results in [DG01]), if the law of $Z_{0}$ is chosen properly, then $Z_{2 n}^{(2)}$ has the same law as $X_{2 n}$ knowing $Y_{\tau: 2 \tau}, \ldots, Y_{(2 n-1) \tau: 2 n \tau}$, hence the title of this Subsection.

Remark 5.2. We have that, for all $k \geq 1, Z_{2 k}^{(2)}$ takes values in $C_{2 k}(\Delta)$ and $Z_{2 k}^{(1)}$ takes values in $C_{2 k-1}(\Delta)$.

We set $\left(Z_{2 k}^{(p)}\right)_{0 \leq k \leq n}$ to be a non-homogeneous Markov chain with $Z_{0}^{(p)}=Z_{0}$ and with kernel $S_{2 n \mid 0}^{\Delta,(p)}, S_{2 n \mid 2}^{\Delta,(p)}, \ldots, S_{2 n \mid 2 n-2}^{\Delta,(p)}$.

We set $U_{2 k+1}=\left(Z_{2 k}^{(2)}, Z_{2 k+2}^{(1)}\right)$ for $k \in\{0,1, \ldots, n-1\}$ and $U_{2 n+1}^{(1)}=Z_{2 n}^{(2)}$. We set $U_{2 k+1}^{(p)}=$ $\left(Z_{2 k}^{(p)(2)}, Z_{2 k+2}^{(p)(1)}\right)$ for $k \in\{0,1,2, \ldots, n-1\}$.

\subsection{New Markov chain}

Lemma 5.3. The sequence $\left(U_{1}, U_{3}, \ldots, U_{2 n-1}, U_{2 n+1}^{(1)}\right)$ is a non-homogeneous Markov chain. The sequence $\left(U_{1}^{(p)}, U_{3}^{(p)}, \ldots, U_{2 n-3}^{(p)}, U_{2 n-1}^{(p)}\right)$ is a non-homogeneous Markov chain.

If $Z_{0}^{(2)}$ is of law $\mu$, then the law of $U_{1}$ is given by, for all $\left(z, z^{\prime}\right)$ in $\mathbb{R}^{2}$,

$$
\mathbb{P}\left(U_{1} \in\left(d z, d z^{\prime}\right)\right)=\int_{x \in \mathbb{R}} S_{2 n \mid 0}^{\Delta}\left((0, z),\left(d z^{\prime}, d x\right)\right) \mu(d z) .
$$

We write $S_{2 n \mid 2 k+1}^{U}$ for the transition kernel between $U_{2 k-1}$ and $U_{2 k+1}$ (for $k=1,2, \ldots, n-1$ ) and $S_{2 n \mid 2 n+1}^{U}$ for the transition between $U_{2 n-1}$ and $U_{2 n+1}^{(1)}$. We write $S_{2 n \mid 2 k+1}^{(p) U}$ for the transition kernel between $U_{2 n \mid 2 k-1}^{(p)}$ and $U_{2 n \mid 2 k+1}^{(p)}($ for $k=1,2, \ldots, n-1)$ 
Proof. We write the proof only for $\left(U_{1}, U_{3}, \ldots, U_{2 n-1}, U_{2 n+1}^{(1)}\right)$, it would be very similar for the sequence $\left(U_{1}^{(p)}, U_{3}^{(p)}, \ldots, U_{2 n-1}^{(p)}\right)$. Let $\varphi$ be a test function (in $\left.\mathcal{C}_{b}^{+}\left(\mathbb{R}^{2}\right)\right)$. For $k \in\{1, \ldots, n-1\}$, we have (for $z_{0}^{(2)}, z_{2}^{(1)}, \ldots, z_{2 k}^{(1)}$ in $\mathbb{R}$ )

$$
\begin{gathered}
\mathbb{E}\left(\varphi\left(U_{2 k+1}\right) \mid U_{1}=\left(z_{0}^{(2)}, z_{2}^{(1)}\right), \ldots, U_{2 k-1}=\left(z_{2 k-2}^{(2)}, z_{2 k}^{(1)}\right)\right)= \\
\mathbb{E}\left(\varphi\left(Z_{2 k}^{(2)}, Z_{2 k+2}^{(1)}\right) \mid Z_{0}^{(2)}=z_{0}^{(2)}, \ldots, Z_{2 k-2}^{(2)}=z_{2 k-2}^{(2)}, Z_{2 k}^{(1)}=z_{2 k}^{(1)}\right)= \\
\mathbb{E}\left(\varphi\left(Z_{2 k}^{(2)}, Z_{2 k+2}^{(1)}\right) \mid Z_{2 k-2}^{(2)}=z_{2 k-2}^{(2)}, Z_{2 k}^{(1)}=z_{2 k}^{(1)}\right),
\end{gathered}
$$

as $S_{2 n \mid 2 k-2}^{\Delta}\left(z_{2 k-2}^{(1)}, z_{2 k-2}^{(2)}, . ..\right)$ does not depend on $z_{2 k-2}^{(1)}$. So the quantity above is equal to, for any $z \in C_{2 k-1}(\Delta)$,

$$
\begin{aligned}
& \int_{\mathbb{R}^{2}} \varphi\left(z_{2 k}^{(2)}, z_{2 k+2}^{(1)}\right) \frac{S_{2 n \mid 2 k-2}^{\Delta}\left(\left(z, z_{2 k-2}^{(2)}\right),\left(z_{2 k}^{(1)}, z_{2 k}^{(2)}\right)\right)}{\int_{\mathbb{R}} S_{2 n \mid 2 k-2}^{\Delta}\left(\left(z, z_{2 k-2}^{(2)}\right),\left(z_{2 k}^{(1)}, z^{\prime}\right)\right) d z^{\prime}} \\
& \quad \times\left(\int_{\mathbb{R}} S_{2 n \mid 2 k}^{\Delta}\left(\left(z_{2 k}^{(1)}, z_{2 k}^{(2)}\right),\left(z_{2 k+2}^{(1)}, z_{2 k+2}^{(2)}\right)\right) d z_{2 k+2}^{(2)}\right) d z_{2 k}^{(2)} d z_{2 k+2}^{(1)} .
\end{aligned}
$$

A similar computation can be made for $\mathbb{E}\left(\varphi\left(U_{2 n+1}^{(1)}\right) \mid U_{1}, \ldots U_{2 n-1}\right)$.

We set, for all $k$,

$$
\begin{gathered}
\epsilon^{\prime}(D, \Delta)=\exp \left[-\left(\frac{B_{2} p_{2,1}^{2}}{2}+\frac{1}{4 \tau}\right)\left(\frac{\Delta}{B_{2}\left(1+p_{2,1}\right)}+D\right)^{2}\right. \\
\left.-\left(\Delta p_{2,1}+3 M\right)\left(\frac{\Delta}{B_{2}\left(1+p_{2,1}\right)}+D\right)-3 \tau\left(\frac{M}{2}+\frac{M^{2}}{4}\right)\right] \\
\epsilon_{k}^{\prime}=\epsilon^{\prime}\left(\left|m_{k}-m_{k-1}\right|, \Delta\right) .
\end{gathered}
$$

Proposition 5.4. For any $k=1,2, \ldots, n$, the Markov kernel $S_{2 n \mid 2 k+1}^{U}$ is $\left(\epsilon_{2 k-1}^{2}\left(\epsilon_{2 k}^{\prime}\right)^{2}\right)$-contracting. For any $k=1,2, \ldots, n-1$, the Markov kernel $S_{2 n \mid 2 k+1}^{(p) U}$ is is $\left(\epsilon_{2 k-1}^{2}\left(\epsilon_{2 k}^{\prime}\right)^{2}\right)$-contracting.

Before going into the proof of the above Proposition, we need the following technical result. We are interested in the bounds appearing in Lemma 4.4. We suppose that $t_{1}, t_{2}, x, z$ in $\mathbb{R}$ are fixed. To simplify the computations, we introduce the following notations:

$$
\begin{gathered}
\left(\begin{array}{c}
t_{1}^{\prime} \\
t_{2}^{\prime}
\end{array}\right)=\left(P^{-1}\right)^{T}\left(\begin{array}{c}
t_{1} \\
t_{2}
\end{array}\right), \\
M_{1}=\frac{2 M\left(\left|1+p_{2,1}\right|\right)}{p_{1,1}} .
\end{gathered}
$$

Lemma 5.5. Suppose that, for some $D \geq 0$,

$$
\begin{gathered}
|x-z| \leq D, \\
\left|2 B_{2}\left(p_{1,2}+1\right) z-t_{2}^{\prime}\right| \leq \Delta
\end{gathered}
$$

then

$$
\begin{aligned}
& \exp \left(-\frac{1}{4 B_{2}}\left(t_{2}^{\prime}-2 B_{2}\left(p_{2,1}+1\right) z\right)^{2}-B_{2} p_{2,1}^{2} D^{2}-p_{2,1} D \Delta\right) \\
& \leq \exp \left(-\frac{1}{4 B_{2}}\left(t_{2}^{\prime}-2 B_{2}\left(p_{2,1} x+z\right)\right)^{2}\right) \\
& \quad \leq \exp \left(-\frac{1}{4 B_{2}}\left(t_{2}^{\prime}-2 B_{2}\left(p_{2,1}+1\right) z\right)^{2}+p_{2,1} D \Delta\right)
\end{aligned}
$$


Proof. We have

$$
\begin{aligned}
& \exp \left(-\frac{1}{4 B_{2}}\left(t_{2}^{\prime}-2 B_{2}\left(p_{2,1} x+z\right)\right)^{2}\right) \\
& \left.=\exp \left(-\frac{1}{4 B_{2}}\left(t_{2}^{\prime}-2 B_{2}\left(p_{2,1}+1\right) z\right)+2 B_{2} p_{2,1}(z-x)\right)^{2}\right) \\
& \leq \exp \left(-\frac{1}{4 B_{2}}\left(t_{2}^{\prime}-2 B_{2}\left(p_{2,1}+1\right) z\right)^{2}-B_{2} p_{2,1}^{2}(z-x)^{2}+\left|p_{2,1}(z-x)\right| \times\left|t_{2}^{\prime}-2 B_{2}\left(p_{2,1}+1\right) z\right|\right) \\
& \leq \exp \left(-\frac{1}{4 B_{2}}\left(t_{2}^{\prime}-2 B_{2}\left(p_{2,1}+1\right) z\right)^{2}+p_{2,1} D \Delta\right)
\end{aligned}
$$

and

$$
\begin{aligned}
& \exp \left(-\frac{1}{4 B_{2}}\left(t_{2}^{\prime}-2 B_{2}\left(p_{2,1} x+z\right)\right)^{2}\right) \\
& \geq \exp \left(-\frac{1}{4 B_{2}}\left(t_{2}^{\prime}-2 B_{2}\left(p_{2,1}+1\right) z\right)^{2}-B_{2} p_{2,1}^{2}(z-x)^{2}-\left|p_{2,1}(z-x)\right| \times\left|t_{2}^{\prime}-2 B_{2}\left(p_{2,1}+1\right) z\right|\right) \\
& \geq \exp \left(-\frac{1}{4 B_{2}}\left(t_{2}^{\prime}-2 B_{2}\left(p_{2,1}+1\right) z\right)^{2}-B_{2} p_{2,1}^{2} D^{2}-p_{2,1} D \Delta\right)
\end{aligned}
$$

Proof of Proposition 5.4. We write the proof in the case $k \in\{1,2, \ldots, n-1\}$ and for $S_{2 n \mid 2 k+1}^{U}$ (the other cases being very similar). Let $\varphi$ be a test function $\left(\right.$ in $\left.\mathcal{C}_{b}^{+}(\mathbb{R})\right)$. By Remark 5.2 , we have that $U_{2 k-1}^{(2)}$ takes its values in $C_{2 k-1}(\Delta)$. We write, for any $z_{2 k-2}^{(2)} \in \mathbb{R}, z_{2 k}^{(1)} \in C_{2 k-1}(\Delta), z \in \mathbb{R}$ (like in the proof of Lemma 5.3)

$$
\begin{aligned}
\mathbb{E}\left(\varphi\left(U_{2 k+1}\right) \mid U_{2 k-1}=\right. & \left.\left(z_{2 k-2}^{(2)}, z_{2 k}^{(1)}\right)\right)= \\
& \int_{\mathbb{R}^{2}} \varphi\left(z_{2 k}^{(2)}, z_{2 k+2}^{(1)}\right) \frac{S_{2 n \mid 2 k-2}^{\Delta}\left(\left(z, z_{2 k-2}^{(2)}\right),\left(z_{2 k}^{(1)}, z_{2 k}^{(2)}\right)\right)}{\int_{\mathbb{R}} S_{2 n \mid 2 k-2}^{\Delta}\left(\left(z, z_{2 k-2}^{(2)}\right),\left(z_{2 k}^{(1)}, z^{\prime}\right)\right) d z^{\prime}} \\
\times & \left(\int_{\mathbb{R}} S_{2 n \mid 2 k}^{\Delta}\left(\left(z_{2 k}^{(1)}, z_{2 k}^{(2)}\right),\left(z_{2 k+2}^{(1)}, z_{2 k+2}^{(2)}\right) d z_{2 k+2}^{(2)}\right) d z_{2 k}^{(2)} d z_{2 k+2}^{(1)} \geq\right.
\end{aligned}
$$

(by Equations (5.5), (5.6))

$$
\begin{gathered}
\int_{\mathbb{R}^{2}} \varphi\left(z_{2 k}^{(2)}, z_{2 k+2}^{(1)}\right) \epsilon_{2 k-1}^{2} \\
\times \frac{\widetilde{R}_{2 n: 2 k+2}^{\Delta}\left(\left(z_{2 k}^{(1)}, z_{2 k}^{(2)}\right), \mathbb{R}^{2}\right) \mathbb{1}_{C_{2 k-1}(\Delta)}\left(z_{2 k}^{(1)}\right) \mathbb{1}_{C_{2 k}(\Delta)}\left(z_{2 k}^{(2)}\right) \psi_{2 k}\left(z_{2 k}^{(1)}, z_{2 k}^{(2)}\right) Q\left(z_{2 k}^{(1)}, z_{2 k}^{(2)}\right)}{\int_{\mathbb{R}} \widetilde{R}_{2 n: 2 k+2}^{\Delta}\left(\left(z_{2 k}^{(1)}, z^{\prime}\right), \mathbb{R}^{2}\right) \mathbb{1}_{C_{2 k-1}(\Delta)}\left(z_{2 k}^{(1)}\right) \mathbb{1}_{C_{2 k}(\Delta)}\left(z^{\prime}\right) \psi_{2 k}\left(z_{2 k}^{(1)}, z^{\prime}\right) Q\left(z_{2 k}^{(1)}, z^{\prime}\right) d z^{\prime}} \\
\times\left(\int_{\mathbb{R}} S_{2 n \mid 2 k}^{\Delta}\left(\left(z_{2 k}^{(1)}, z_{2 k}^{(2)}\right),\left(z_{2 k+2}^{(1)}, z_{2 k+2}^{(2)}\right) d z_{2 k+2}^{(2)}\right) d z_{2 k}^{(2)} d z_{2 k+2}^{(1)}\right.
\end{gathered}
$$

From Lemma 2.2 and using the same kind of computation as in the proof of Lemma 4.4 and Equation (4.15), we get, for all $z_{2 k}$ such that $z_{2 k}^{(2)} \in C_{2 k}(\Delta)$,

$$
\begin{aligned}
& \psi_{2 k}\left(z_{2 k}^{(1)}, z_{2 k}^{(2)}\right) \geq \sigma_{1} \sigma_{2} e^{-2 M\left|z_{2 k}^{(1)}-z_{2 k}^{(2)}\right|-\tau\left(M+\frac{M^{2}}{2}\right)} \\
\times & \exp \left(-A_{2}\left(z_{2 k}^{(1)}\right)^{2}-B_{2}\left(z_{2 k}^{(2)}\right)^{2}+C_{1} z_{2 k}^{(1)} z_{2 k}^{(2)}+A_{1}^{y_{(2 k-1) \tau:(2 k) \tau}} z_{2 k}^{(1)}+B_{1}^{y_{(2 k-1) \tau:(2 k) \tau}} z_{2 k}^{(2)}+C_{0}^{y_{(2 k-1) \tau:(2 k) \tau}}\right) \\
= & \sigma_{1} \sigma_{2} e^{-2 M\left|z_{2 k}^{(1)}-z_{2 k}^{(2)}\right|-\tau\left(M+\frac{M^{2}}{2}\right)} \times \exp \left[-\frac{1}{4 A_{2}}\left(\frac{A_{1}^{y_{(2 k-1) \tau:(2 k) \tau}}}{p_{1,1}}-\frac{p_{2,1} B_{1}^{y_{(2 k-1) \tau:(2 k) \tau}}}{p_{1,1}}-2 A_{2} p_{1,1} z_{2 k}^{(1)}\right)^{2}\right.
\end{aligned}
$$




$$
\begin{aligned}
& -\frac{1}{4 B_{2}}\left(B_{1}^{y_{(2 k-1) \tau:(2 k) \tau}}-2 B_{2}\left(p_{2,1} z_{2 k}^{(1)}+z_{2 k}^{(2)}\right)\right)^{2}+C_{0}^{y_{(2 k-1) \tau:(2 k) \tau}} \\
& \left.+\frac{1}{4}\left(A_{1}^{y_{(2 k-1) \tau:(2 k) \tau}}, B_{1}^{y(2 k-1) \tau:(2 k) \tau}\right) \kappa^{-1}\left(A_{1}^{y(2 k-1) \tau:(2 k) \tau}, B_{1}^{y(2 k-1) \tau:(2 k) \tau}\right)^{T}\right] \\
& \geq \text { (by Lemma 5.5) } \sigma_{1} \sigma_{2} e^{-2 M\left|z_{2 k}^{(1)}-z_{2 k}^{(2)}\right|+C_{0}^{y(2 k-1) \tau:(2 k) \tau}} \times e^{-\tau\left(M+\frac{M^{2}}{2}\right)} \\
& \times \exp \left[-\frac{1}{4 A_{2}}\left(\frac{A_{1}^{y_{(2 k-1) \tau:(2 k) \tau}}}{p_{1,1}}-\frac{p_{2,1} B_{1}^{y_{(2 k-1) \tau:(2 k) \tau}}}{p_{1,1}}-2 A_{2} p_{1,1} z_{2 k}^{(1)}\right)^{2}\right. \\
& -\frac{1}{4 B_{2}}\left(B_{1}^{y(2 k-1) \tau:(2 k) \tau}-2 B_{2}\left(p_{2,1}+1\right) z_{2 k}^{(2)}\right)^{2}-B_{2} p_{2,1}^{2}\left(z_{2 k}^{(1)}-z_{2 k}^{(2)}\right)^{2}-\Delta p_{2,1}\left|z_{2 k}^{(1)}-z_{2 k}^{(2)}\right| \\
& \left.+\frac{1}{4}\left(A_{1}^{y_{(2 k-1) \tau:(2 k) \tau}}, B_{1}^{y_{(2 k-1) \tau:(2 k) \tau}}\right) \kappa^{-1}\left(A_{1}^{y_{(2 k-1) \tau:(2 k) \tau}}, B_{1}^{y_{(2 k-1) \tau:(2 k) \tau}}\right)^{T}\right] .
\end{aligned}
$$

We set

$$
\begin{gathered}
\psi_{2 k}^{(1)}(x)=\exp \left(-\frac{1}{4 A_{2}}\left(\frac{A_{1}^{y(2 k-1) \tau:(2 k) \tau}}{p_{1,1}}-\frac{p_{2,1} B_{1}^{y_{(2 k-1) \tau:(2 k 2) \tau}}}{p_{1,1}}-2 A_{2} p_{1,1} x\right)^{2}\right), \\
\psi_{2 k}^{(2)}(x)=\exp \left(-\frac{1}{4 B_{2}}\left(B_{1}^{y_{(2 k-1) \tau:(2 k) \tau}}-2 B_{2}\left(p_{2,1}+1\right) x\right)^{2}\right)
\end{gathered}
$$

In the same way as above:

$$
\begin{aligned}
& \psi_{2 k}\left(z_{2 k}^{(1)}, z_{2 k}^{(2)}\right) \leq \sigma_{1} \sigma_{2} e^{2 M\left|z_{2 k}^{(1)}-z_{2 k}^{(2)}\right|+\tau\left(M+\frac{M^{2}}{2}\right)+C_{0}^{y(2 k-1) \tau:(2 k) \tau}} \psi_{2 k}^{(1)}\left(z_{2 k}^{(1)}\right) \psi_{2 k}^{(2)}\left(z_{2 k}^{(2)}\right) \\
\times & \exp \left(\Delta p_{2,1}\left|z_{2 k}^{(1)}-z_{2 k}^{(2)}\right|+\frac{1}{4}\left(A_{1}^{y(2 k-1) \tau:(2 k) \tau}, B_{1}^{y(2 k-1) \tau:(2 k) \tau}\right) \kappa^{-1}\left(A_{1}^{y_{(2 k-1) \tau:(2 k) \tau}}, B_{1}^{y_{(2 k-1) \tau:(2 k) \tau}}\right)^{T}\right) .
\end{aligned}
$$

From Lemma 2.1, we get for all $z_{2 k}^{(1)} \in C_{2 k-1}(\Delta), z_{2 k}^{(2)} \in C_{2 k}(\Delta)$,

$$
\begin{array}{r}
\frac{1}{\sqrt{2 \pi \tau}} \exp \left(-\frac{1}{2 \tau}\left(\frac{\Delta}{B_{2}\left(1+p_{2,1}\right)}+D_{2 k}\right)^{2}-M\left(\frac{\Delta}{B_{2}\left(1+p_{2,1}\right)}+D_{2 k}\right)-\tau\left(\frac{M}{2}+\frac{M^{2}}{2}\right)\right) \\
\leq Q\left(z_{2 k}^{(1)}, z_{2 k}^{(2)}\right) \leq \frac{1}{\sqrt{2 \pi \tau}} \exp \left(M\left(\frac{\Delta}{B_{2}\left(1+p_{2,1}\right)}+D_{2 k}\right)+\frac{M \tau}{2}\right)
\end{array}
$$

Looking back at (5.9), we get

$$
\begin{aligned}
& \mathbb{E}\left(\varphi\left(U_{2 k+1}\right) \mid U_{2 k-1}=\left(z_{2 k-2}^{(2)}, z_{2 k}^{(1)}\right)\right) \geq \\
& \int_{\mathbb{R}^{2}} \varphi\left(z_{2 k}^{(2)}, z_{2 k+2}^{(1)}\right) \epsilon_{2 k-1}^{2}\left(\epsilon_{2 k}^{\prime}\right)^{2} \frac{\widetilde{R}_{2 n: 2 k+2}^{\Delta}\left(\left(z_{2 k}^{(1)}, z_{2 k}^{(2)}\right), \mathbb{R}^{2}\right) \mathbb{1}_{C_{2 k-1}(\Delta)}\left(z_{2 k}^{(1)}\right) \mathbb{1}_{C_{2 k}(\Delta)}\left(z_{2 k}^{(2)}\right) \psi_{2 k}^{(2)}\left(z_{2 k}^{(2)}\right)}{\int_{\mathbb{R}} \widetilde{R}_{2 n: 2 k+2}^{\Delta}\left(\left(z_{2 k}^{(1)}, z^{\prime}\right), \mathbb{R}^{2}\right) \mathbb{1}_{C_{2 k-1}(\Delta)}\left(z_{2 k}^{(1)}\right) \mathbb{1}_{C_{2 k}(\Delta)}\left(z_{2 k}^{(2)}\right) \psi_{2 k}^{(2)}\left(z^{\prime}\right) d z^{\prime}} \\
& \quad \times\left(\int_{\mathbb{R}} S_{2 n \mid 2 k}^{\Delta}\left(\left(z_{2 k}^{(1)}, z_{2 k}^{(2)}\right),\left(z_{2 k+2}^{(1)}, z_{2 k+2}^{(2)}\right) d z_{2 k+2}^{(2)}\right) d z_{2 k}^{(2)} d z_{2 k+2}^{(1)} .\right.
\end{aligned}
$$

As $R_{2 n: 2 k+2}\left(\left(z_{2 k}^{(1)}, z^{\prime}\right),.\right)$ and $S_{2 n \mid 2 k}^{\Delta}\left(\left(z_{2 k}^{(1)}, z^{\prime}\right),.\right)$ do not depend on $z_{2 k}^{(1)}$ for any $z^{\prime}$, we get that $S_{2 n \mid 2 k+1}^{U}$ is $\left(1-\epsilon_{2 k-1}^{2}\left(\epsilon_{2 k}^{\prime}\right)^{2}\right)$-contracting (remember Section 3.1).

\subsection{New representation}

Proposition 5.6. Let $n \geq 1$. If we suppose that $Z_{0}^{(2)}$ is of law $\psi_{2 n \mid 0}^{\Delta}(0,.) \bullet \mu$, then, for all test function $\varphi\left(\right.$ in $\left.\mathcal{C}_{b}^{+}(\mathbb{R})\right)$,

$$
\frac{\mathbb{E}\left(\varphi\left(U_{2 n+1}^{(1)}\right) \prod_{1 \leq i \leq n} \psi_{2 i-1}^{\Delta}\left(U_{2 i-1}\right)\right)}{\mathbb{E}\left(\prod_{1 \leq i \leq n} \psi_{2 i-1}^{\Delta}\left(U_{2 i-1}\right)\right)}=\left(\bar{R}_{2 n}^{\Delta} \bar{R}_{2 n-1}^{\Delta} \ldots \bar{R}_{1}^{\Delta}(\mu)\right)(\varphi) .
$$


If we suppose that $Z_{0}^{(2)}$ is of law $\psi_{2 n \mid 0}^{\Delta,(p)}(0,.) \bullet \mu$, then, for all test function $\varphi\left(\right.$ in $\left.\mathcal{C}_{b}^{+}(\mathbb{R})\right)$,

$$
\frac{\mathbb{E}\left(\varphi\left(U_{2 n-1}^{(p)(2)}\right) \prod_{1 \leq i \leq n} \psi_{2 i-1}^{\Delta}\left(U_{2 i-1}^{(p)}\right)\right)}{\mathbb{E}\left(\prod_{1 \leq i \leq n} \psi_{2 i-1}^{\Delta}\left(U_{2 i-1}^{(p)}\right)\right)}=\left(\bar{R}_{2 n-1}^{\Delta} \bar{R}_{2 n-2}^{\Delta} \ldots \bar{R}_{1}^{\Delta}(\mu)\right)(\varphi) .
$$

Remark 5.7. Recall that we are working with a fixed observation $\left(Y_{s}\right)_{s \geq 0}=\left(y_{s}\right)_{s \geq 0}$. The above Proposition tells that, for all $n, \bar{R}_{n}^{\Delta} \bar{R}_{n-1}^{\Delta} \ldots \bar{R}_{1}^{\Delta}(\mu)$ can be written as the $n$-th term of a FeynmanKac sequence based on mixing kernels (by Proposition 5.4). We can apply Proposition 3.1 to this Feynman-Kac sequence. This representation and this result are also true for a measure $\bar{R}_{n}^{\Delta} \bar{R}_{n-1}^{\Delta} \ldots \bar{R}_{k}^{\Delta}(\eta)$ for any $k \leq n, \eta$ probability measure on $\mathbb{R}$.

Proof. We write the proof only for Equation (5.10). The computation leading to Equation (5.11) would be very similar. It would simplify nicely because we replace $\psi_{2 n}^{\Delta}$ by 1 in the definition of the $S_{2 n \mid \ldots}^{\Delta(p)}, \psi_{2 n \mid \ldots}^{\Delta(p)}$.

We have, for any test function $\varphi\left(\right.$ in $\left.\mathcal{C}_{b}^{+}(\mathbb{R})\right)$,

$$
\begin{aligned}
& \mathbb{E}\left(\varphi\left(U_{2 n+1}^{(1)}\right) \prod_{1 \leq i \leq n} \psi_{2 i-1}^{\Delta}\left(U_{2 i-1}\right)\right)= \\
& \int_{\mathbb{R} \times\left(\mathbb{R}^{2}\right)^{n}} \varphi\left(z_{2 n}^{(2)}\right) \prod_{0 \leq k \leq n-1}\left[S_{2 n \mid 2 k}^{\Delta}\left(z_{2 k}, z_{2 k+2}\right) \psi_{2 k+1}^{\Delta}\left(z_{2 k}^{(2)}, z_{2 k+2}^{(1)}\right)\right] \\
& \delta_{0}\left(d z_{0}^{(1)}\right)\left(\psi_{2 n \mid 0}^{\Delta}(0, .) \bullet \mu\right)\left(d z_{0}^{(2)}\right) d z_{2} \ldots d z_{2 n}= \\
& \int_{\mathbb{R} \times\left(\mathbb{R}^{2}\right)^{n}} \varphi\left(z_{2 n}^{(2)}\right) \prod_{0 \leq k \leq n-1}\left[\frac{\psi_{2 n \mid 2 k+2}^{\Delta}\left(z_{2 k+2}\right)}{\psi_{2 n \mid 2 k}^{\Delta}\left(z_{2 k}\right)} \widetilde{R}_{2 k+2}^{\Delta}\left(z_{2 k}, d z_{2 k+2}\right) \psi_{2 k+1}^{\Delta}\left(z_{2 k}^{(2)}, z_{2 k+2}^{(1)}\right)\right] \\
& \times \delta_{0}\left(d z_{0}^{(1)}\right)\left(\psi_{2 n \mid 0}^{\Delta}(0, .) \bullet \mu\right)\left(d z_{0}^{(2)}\right) d z_{2} \ldots d z_{2 n}= \\
& \int_{\mathbb{R} \times\left(\mathbb{R}^{2}\right)^{n}} \varphi\left(z_{2 n}^{(2)}\right) \prod_{0 \leq k \leq n-1}\left[\widetilde{R}_{2 k+2}^{\Delta}\left(z_{2 k}, d z_{2 k+2}\right) \psi_{2 k+1}^{\Delta}\left(z_{2 k}^{(2)}, z_{2 k+2}^{(1)}\right)\right] \\
& \times \frac{1}{\mu\left(\psi_{2 n \mid 0}^{\Delta}(0, .)\right)} \delta_{0}\left(d z_{0}^{(1)}\right) \mu\left(d z_{0}^{(2)}\right) d z_{2} \ldots d z_{2 n}= \\
& \int_{\mathbb{R} \times\left(\mathbb{R}^{2}\right)^{n}} \varphi\left(z_{2 n}^{(2)}\right) \\
& \prod_{0 \leq k \leq n-1}\left[\psi_{2 k+2}^{\Delta}\left(z_{2 k+2}^{(1)}, z_{2 k+2}^{(2)}\right) \psi_{2 k+1}^{\Delta}\left(z_{2 k}^{(2)}, z_{2 k+2}^{(1)}\right) \widetilde{Q}^{2}\left(z_{2 k}, d z_{2 k+2}\right)\right] \\
& \times \frac{1}{\mu\left(\psi_{2 n \mid 0}^{\Delta}(0, .)\right)} \delta_{0}\left(d z_{0}^{(1)}\right) \mu\left(d z_{0}^{(2)}\right) d z_{2} \ldots d z_{2 n},
\end{aligned}
$$

which proves the desired result (recall Equation (3.11)).

\section{$6 \quad$ Stability results}

In this section, the observations are non longer fixed.

\subsection{Stability of the truncated filter}

We show here that a product of coefficients $\tau$. decays geometrically in expectation (see the Lemma below). These coefficients are the contraction coefficients of the operators $S^{U}, S^{U,(p)}$, which are related to the truncated filter through Proposition 5.6. This is why we say that the result below means the stability of the truncated filter.

We set, for all $t$ in $\mathbb{R}, k \geq 1$,

$$
\tau(t, \Delta)=1-\left(\epsilon^{\prime}(t, \Delta) \epsilon(t, \Delta)\right)^{2},
$$




$$
\tau_{k}=1-\left(\epsilon_{k}^{\prime} \epsilon_{k-1}\right)^{2} .
$$

We set, for $L>0$,

$$
\widetilde{\alpha}(L)=\frac{192 C \sqrt{\tau}}{L \sqrt{\pi}} \exp \left(-\frac{1}{2}\left(\frac{L}{12 C \sqrt{2 \tau}}\right)^{2}\right) \text {. }
$$

We fix $L>0$ such that

$$
L>3\left|m_{0}\right|+3 C M \tau \text { and } \widetilde{\alpha}(L) \leq \frac{1}{4} .
$$

We set

$$
\rho=\frac{\tau(L, \Delta)+\sqrt{\tau(L, \Delta)^{2}+4 \widetilde{\alpha}(L)(1-\tau(L, \Delta))}}{2} .
$$

Lemma 6.1. For $0 \leq k \leq n-1$, we have

$$
\begin{gathered}
\mathbb{E}\left(\tau_{2 n+1} \tau_{2 n-1} \ldots \tau_{2 k+3} \mid \mathcal{F}_{0:(2 k+1) \tau}\right) \leq\left(1-\frac{\epsilon(L, \Delta)^{2} \epsilon^{\prime}(L, \Delta)^{2}}{2}\right)^{\left\lceil\frac{(n-k-2)+}{2}\right\rceil}, \\
\mathbb{E}\left(\tau_{2 n} \tau_{2 n-2} \ldots \tau_{2 k+2} \mid \mathcal{F}_{0: 2 k \tau}\right) \leq\left(1-\frac{\epsilon(L, \Delta)^{2} \epsilon^{\prime}(L, \Delta)^{2}}{2}\right)^{\left\lceil\frac{(n-k-2)+}{2}\right\rceil} .
\end{gathered}
$$

Proof. We only write the proof of the second Equation above (the proof of the other equation is very similar). We take $L>0$ and we set

$$
\theta_{2 k}= \begin{cases}\tau(L, \Delta) & \text { if }\left|m_{2 k}-m_{2 k-1}\right|<L \text { and }\left|m_{2 k-1}-m_{2 k-2}\right|<L \\ 1 & \text { otherwise. }\end{cases}
$$

For all $k$, we have $\tau_{2 k} \leq \theta_{2 k}$. For any $k \geq 1,\left|m_{k}-m_{k-1}\right|$ is a function of $Y_{(k-2)+\tau: k \tau}$. So, for all $k, \theta_{2 k}$ is a function of $Y_{(2 k-3)_{+} \tau: 2 k \tau}$ We fix $k \geq 0$ and we define, for $n \geq 0$,

$$
e_{2 n \mid 2 k+2}= \begin{cases}\mathbb{E}\left(\theta_{2 n} \theta_{2 n-2} \ldots \theta_{2 k+2} \mid \mathcal{F}_{2 k \tau}\right) & \text { if } k \leq n-1, \\ 1 & \text { otherwise. }\end{cases}
$$

We suppose now that $n \geq k+2$. We then have

$$
e_{2 n \mid 2 k+2}=\mathbb{E}\left(\mathbb{E}\left(\theta_{2 n} \theta_{2 n-2} \mid \mathcal{F}_{(2 n-3) \tau}\right) \theta_{2 n-4} \ldots \theta_{2 k+2} \mid \mathcal{F}_{2 k \tau}\right)
$$

and

$$
\begin{aligned}
& \mathbb{E}\left(\theta_{2 n} \theta_{2 n-2} \mid \mathcal{F}_{(2 n-3) \tau}\right) \\
& =\mathbb{E}\left(\theta_{2 n-2}\left(1-\mathbb{1}_{[0, L)}\left(D_{2 n}\right) \mathbb{1}_{[0, L)}\left(D_{2 n-1}\right)\right)+\tau(L, \Delta) \theta_{2 n-2} \mathbb{1}_{[0, L)}\left(D_{2 n}\right) \mathbb{1}_{[0, L)}\left(D_{2 n-1}\right) \mid \mathcal{F}_{(2 n-3) \tau}\right) \\
& =\mathbb{E}\left(\theta_{2 n-2} \tau(L, \Delta)+(1-\tau(L, \Delta)) \theta_{2 n-2}\left(1-\mathbb{1}_{[0, L)}\left(D_{2 n}\right) \mathbb{1}_{[0, L)}\left(D_{2 n-1}\right)\right) \mid \mathcal{F}_{(2 n-3) \tau}\right) \\
& \leq \tau(L, \Delta) \mathbb{E}\left(\theta_{2 n-2} \mid \mathcal{F}_{(2 n-3) \tau}\right)+(1-\tau(L, \Delta))\left[\mathbb{P}\left(\left|m_{2 n}-m_{2 n-1}\right| \geq L \mid \mathcal{F}_{(2 n-3) \tau}\right)\right. \\
& \left.+\mathbb{P}\left(\left|m_{2 n-1}-m_{2 n-2}\right| \geq L \mid \mathcal{F}_{(2 n-3) \tau}\right)\right]
\end{aligned}
$$

Using Equation (4.21), we get

$$
\begin{gathered}
\mathbb{E}\left(\theta_{2 n} \theta_{2 n-2} \mid \mathcal{F}_{(2 n-3) \tau}\right) \leq \tau(L, \Delta) \mathbb{E}\left(\theta_{2 n-2} \mid \mathcal{F}_{(2 n-3) \tau}\right) \\
+(1-\tau(L, \Delta))\left(\mathbb{P}\left(C \mathcal{V}_{(2 n-2) \tau, 2 n \tau} \geq \frac{L}{3} \mid \mathcal{F}_{(2 n-3) \tau}\right)+\mathbb{P}\left(C \mathcal{W}_{(2 n-2) \tau, 2 n \tau}\left(1+\frac{1}{\theta}\right) \geq \frac{L}{3} \mid \mathcal{F}_{(2 n-3) \tau}\right)\right) \\
+(1-\tau(L, \Delta))\left(\mathbb{P}\left(C \mathcal{V}_{(2 n-3) \tau,(2 n-1) \tau} \geq \frac{L}{3} \mid \mathcal{F}_{(2 n-3) \tau}\right)+\mathbb{P}\left(C \mathcal{W}_{(2 n-3) \tau,(2 n-1) \tau}\left(1+\frac{1}{\theta}\right) \geq \frac{L}{3} \mid \mathcal{F}_{(2 n-3) \tau}\right)\right) \\
\leq \tau(L, \Delta) \mathbb{E}\left(\theta_{2 n-2} \mid \mathcal{F}_{(2 n-3) \tau}\right)+4(1-\tau(L, \Delta)) \mathbb{P}\left(C \mathcal{V}_{0,2 \tau} \geq \frac{L}{6}\right)
\end{gathered}
$$


(like in Equations (4.34), (4.35))

$$
\begin{aligned}
& \leq \tau(L, \Delta) \mathbb{E}\left(\theta_{2 n-2} \mid \mathcal{F}_{(2 n-3) \tau}\right)+8(1-\tau(L, \Delta)) \mathbb{P}\left(2 C\left|W_{2 \tau}\right| \geq \frac{L}{6}\right) \\
&(\text { using Equation }(4.36)) \\
& \leq \tau(L, \Delta) \mathbb{E}\left(\theta_{2 n-2} \mid \mathcal{F}_{(2 n-3) \tau}\right)+(1-\tau(L, \Delta)) \widetilde{\alpha}(L) .
\end{aligned}
$$

The constant $\rho$ is the positive root of the polynomial $X^{2}-\tau(L, \Delta) X-(1-\tau(L, \Delta)) \widetilde{\alpha}(L)$. So we have

$$
1>\rho=\tau(L, \Delta)+\frac{1}{\rho}(1-\tau(L, \Delta)) \widetilde{\alpha}(L) \geq \tau(L, \Delta)+(1-\tau(L, \Delta)) \widetilde{\alpha}(L) .
$$

So, we have

$$
e_{2 n \mid 2 k+2} \leq \tau(L, \Delta) e_{2 n-2 \mid 2 k+2}+(1-\tau(L, \Delta)) \widetilde{\alpha}(L) e_{2 n-4 \mid 2 k+2} \leq \rho \times \sup \left(e_{2 n-2 \mid 2 k+2}, e_{2 n-4 \mid 2 k+2}\right) .
$$

Suppose now that $k$ is fixed. We have

$$
e_{2 k+2 \mid 2 k+2} \leq 1, e_{2 k+4 \mid 2 k+2} \leq 1 .
$$

So, by recurrence,

$$
e_{2 n \mid 2 k+2} \leq \rho^{\left\lceil\frac{(n-k-2)_{+}}{2}\right\rceil} .
$$

As $\widetilde{\alpha}(L) \leq 1 / 4$, we have

$$
\rho \leq \frac{1}{2}\left(\tau(L, \Delta)+\sqrt{\tau(L, \Delta)^{2}+1-\tau(L, \Delta)}\right) \leq \frac{\tau(L, \Delta)+1}{2}=1-\frac{\left(\epsilon(L, \Delta)^{2} \epsilon^{\prime}(L, \Delta)\right)^{2}}{2} .
$$

Proof of Lemma 1.4. We write the proof in the case where $n$ and $k$ are even. If $k$ was even and $n$ was odd, we would have to use the operators $S_{\ldots}^{(p) U}$. If $k$ was odd, the proof would be very similar but would require to introduce new and heavy notations.

By Proposition 5.6, Remark 5.7 and Equation (5.7), we have, for all $\mu$ in $\mathcal{P}(\mathbb{R})$ and all test function $\varphi$ in $\mathcal{C}_{b}^{+}(\mathbb{R})$,

$$
\begin{aligned}
&\left(\bar{R}_{n}^{\Delta} \bar{R}_{n-1}^{\Delta} \ldots \bar{R}_{k+1}^{\Delta}(\mu)\right)(\varphi) \propto \int \varphi\left(u_{n+1}^{(1)}\right)\left(\prod_{i=1}^{(n-k) / 2} \psi_{k+2 i-1}^{\Delta}\left(u_{k+2 i-1}\right)\right) \\
& \times\left(\prod_{i=1}^{(n-k-2) / 2} S_{n \mid k+2 i+1}^{U}\left(u_{k+2 i-1}, d u_{k+2 i+1}\right)\right) \\
& \times S_{n \mid n+1}^{U}\left(u_{n-1}, d u_{n+1}^{(1)}\right)\left(\int_{z^{\prime} \in \mathbb{R}} S_{n \mid k}^{\Delta}\left(\left(0, u_{k+1}^{(1)}\right),\left(d u_{k+1}^{(2)}, d z^{\prime}\right)\right)\left(\psi_{n \mid k}^{\Delta}(0, .) \bullet \mu\right)\left(d u_{k+1}^{(1)}\right),\right.
\end{aligned}
$$

where we integrate over $u_{k+1}^{(1)} \in \mathbb{R}, u_{k+1}, u_{k+3}, \ldots, u_{n-1} \in \mathbb{R}^{2}, u_{n+1}^{(1)} \in \mathbb{R}$.

By Proposition 5.4, we know that $S_{n \mid k+2 i+1}^{U}$ is $\left(\epsilon_{k+2 i-1}^{2}\left(\epsilon_{k+2 i}^{\prime}\right)^{2}\right)$-mixing for all $i$ in $\{1,2, \ldots, 1+$ $(n-k) / 2\}$. We now apply Proposition 3.1 with the $S_{n \mid k+2 i+1}^{U}$ playing the roles of the $\mathfrak{Q} \ldots$ and the $\psi_{k+2 i-1}^{\Delta}$ playing the roles of the $\Psi_{\ldots .}$. By Equations (3.12), (3.13), we then have, for all $\mu$ and $\mu^{\prime}$ in $\mathcal{P}(\mathbb{R})$,

$$
\begin{aligned}
& \left\|\bar{R}_{n}^{\Delta} \bar{R}_{n-1}^{\Delta} \ldots \bar{R}_{k+1}^{\Delta}(\mu)-\bar{R}_{n}^{\Delta} \bar{R}_{n-1}^{\Delta} \ldots \bar{R}_{k+1}^{\Delta}\left(\mu^{\prime}\right)\right\| \\
& \quad \leq \prod_{i=1}^{(n-k) / 2}\left(1-\epsilon_{k+2 i-1}^{2}\left(\epsilon_{k+2 i}^{\prime}\right)^{2}\right) \times 2 \inf \left(1, \frac{\left\|\psi_{n \mid k}^{\Delta}(0, .) \bullet \mu-\psi_{n \mid k}^{\Delta}(0, .) \bullet \mu^{\prime}\right\|}{\epsilon_{k+1}^{2}\left(\epsilon_{k+2}^{\prime}\right)^{2}}\right) .
\end{aligned}
$$


By Equations (5.4), (3.12), (3.13), we have

$$
\begin{aligned}
\left\|\psi_{n \mid k}^{\Delta}(0, .) \bullet \mu-\psi_{n \mid k}^{\Delta}(0, .) \bullet \mu^{\prime}\right\| & \leq 2 \inf \left(1, \frac{\left\|\psi_{n \mid k}^{\Delta}(0, .)\right\|_{\infty}}{\left\langle\mu, \psi_{n \mid k}^{\Delta}(0, .)\right\rangle}\left\|\mu-\mu^{\prime}\right\|\right) \\
& \leq 2 \inf \left(1, \frac{\left\|\mu-\mu^{\prime}\right\|}{\epsilon_{k+1}^{2}}\right) .
\end{aligned}
$$

From which we get the result.

\subsection{Approximation of the optimal filter by the truncated filter}

We recall that " $\frac{\preceq}{\Delta}$ is defined in Definition 2.5.

Proof of Proposition 1.5. We write the proof only for Equation (1.4), the proof for Equation (1.5) being very similar. We have

$$
\left\|\pi_{n \tau}-\pi_{n \tau}^{\Delta}\right\| \leq\left\|\pi_{n \tau}-\bar{R}_{n}^{\Delta}\left(\pi_{(n-1) \tau}\right)\right\|+\sum_{1 \leq k \leq n-1}\left\|\bar{R}_{n: k+1}^{\Delta}\left(\pi_{k \tau}\right)-\bar{R}_{n: k+1}^{\Delta}\left(\bar{R}_{k}^{\Delta}\left(\pi_{(k-1) \tau}\right)\right)\right\| .
$$

Let us fix $k \in\{1,2, \ldots, n-1\}$. From Lemma 1.4, we get

$$
\begin{aligned}
& \mathbb{E}\left(\left\|\bar{R}_{n: k+1}^{\Delta}\left(\pi_{k \tau}\right)-\bar{R}_{n: k+1}^{\Delta}\left(\bar{R}_{k}^{\Delta}\left(\pi_{(k-1) \tau}\right)\right)\right\|\right) \\
& \leq \mathbb{E}\left(\mathbb{E}\left(\prod_{3 \leq i \leq\left\lfloor\frac{n-k}{2}\right\rfloor}\left(1-\left(\epsilon_{k+2 i-1}^{2}\left(\epsilon_{k+2 i}^{\prime}\right)^{2}\right)\right) \mid \mathcal{F}_{(k+2) \tau}\right) \times 2 \inf \left(1, \frac{\left\|\pi_{k \tau}-\bar{R}_{k}^{\Delta}\left(\pi_{(k-1) \tau}\right)\right\|}{\left(\epsilon_{k+2}^{\prime}\right)^{2} \epsilon_{k+1}^{4}}\right)\right),
\end{aligned}
$$

with the convention that a product over indexes in the null set is equal to one. From Lemma 6.1, we get

$$
\begin{aligned}
\mathbb{E}\left(\left\|\bar{R}_{n: k+1}^{\Delta}\left(\pi_{k \tau}\right)-\bar{R}_{n: k+1}^{\Delta}\left(\bar{R}_{k}^{\Delta}\left(\pi_{(k-1) \tau}\right)\right)\right\|\right) \leq & 1 \\
& \left.=\frac{\left(\epsilon(L, \Delta)^{2} \epsilon^{\prime}(L, \Delta)\right)^{2}}{2}\right)^{\left\lceil\frac{1}{2}\left(\left\lfloor\frac{n-k}{2}\right\rfloor-4\right)_{+}\right\rceil} \\
& \times 2 \mathbb{E}\left(\inf \left(1, \frac{\left\|\pi_{k \tau}-\bar{R}_{k}^{\Delta}\left(\pi_{(k-1) \tau}\right)\right\|}{\left(\epsilon_{k+2}^{\prime}\right)^{2} \epsilon^{4} k+1}\right)\right) .
\end{aligned}
$$

As in [OR05], p. 434, we can bound

$$
\inf \left(1, \frac{\left\|\pi_{n \tau}-\bar{R}_{k}^{\Delta}\left(\pi_{(k-1) \tau}\right)\right\|}{\left(\epsilon_{k+2}^{\prime}\right)^{2} \epsilon_{k+1}^{4}}\right) \leq \inf \left(1, \frac{T(\Delta)}{\left(\epsilon_{k+2}^{\prime}\right)^{4} \epsilon_{k+1}^{8}}\right)+\inf \left(1, \frac{\left\|\pi_{n \tau}-\bar{R}_{k}^{\Delta}\left(\pi_{(k-1) \tau}\right)\right\|^{2}}{T(\Delta)}\right) .
$$

We have, if $\Delta$ satisfies the assumption of Proposition 4.7,

$$
\begin{gathered}
\mathbb{E}\left(\inf \left(1, \frac{\left\|\pi_{k \tau}-\bar{R}_{k}^{\Delta}\left(\pi_{(k-1) \tau}\right)\right\|^{2}}{T(\Delta)}\right)\right) \\
=\mathbb{E}\left(\frac{\left\|\pi_{k \tau}-\bar{R}_{k}^{\Delta}\left(\pi_{(k-1) \tau}\right)\right\|^{2}}{T(\Delta)} \mathbb{1}_{[0,1]}\left(\frac{\left\|\pi_{k \tau}-\bar{R}_{k}^{\Delta}\left(\pi_{(k-1) \tau}\right)\right\|^{2}}{T(\Delta)}\right)\right) \\
+\mathbb{P}\left(\frac{\left\|\pi_{k \tau}-\bar{R}_{k}^{\Delta}\left(\pi_{(k-1) \tau}\right)\right\|^{2}}{T(\Delta)}>1\right) \\
\leq \frac{2}{\sqrt{T(\Delta)}} \mathbb{E}\left(\left\|\pi_{k \tau}-\bar{R}_{k}^{\Delta}\left(\pi_{(k-1) \tau}\right)\right\|\right)
\end{gathered}
$$


We look now at the term $\inf \left(1, T(\Delta)\left(\epsilon_{k+2}^{\prime}\right)^{-4} \epsilon_{k+1}^{-8}\right)$. Using Equations (4.4), (4.22), (4.23), (7.12), (7.17), (7.18), (7.20) and the remarks below Equation (4.4), we have, for all $k$,

$$
\begin{aligned}
& T(\Delta) \underset{\Delta, c}{\preceq} \frac{h^{-1+\iota} \tau^{\iota-\frac{1}{2}}}{\Delta} \exp \left(-\frac{1}{2}\left(\frac{\tau^{\frac{1}{2}-\iota} h^{1-\iota} \Delta}{12 C \sqrt{2}}\right)^{2}\right) \\
& \quad+(M+\sqrt{h}) \frac{h e^{26 M^{2} \tau+\frac{7 \tau M}{2}+40 M^{2} / h}}{\Delta \theta^{3 / 2}} \exp \left(-\frac{1}{4 B_{2}} d(\Delta)^{2}\right) C_{1}^{\prime}(h, \tau)+e^{-C_{0} \Delta^{2}}
\end{aligned}
$$

and using Equations (4.26), (4.27), (5.3),

$$
\begin{aligned}
& \left.\epsilon(D, \Delta)^{-1}=\exp \left(\frac{\left(D+\frac{\Delta}{B_{2}\left(1+p_{2,1}\right)}\right)^{2}}{2 \tau}-\frac{\left(\left(D-\frac{\Delta}{B_{2}\left(1+p_{2,1}\right)}\right)\right.}{2 \tau}\right)^{2}\right) e^{2 M\left(D+\frac{\Delta}{B_{2}\left(1+p_{2,1}\right.}\right)+\left(\tau+\frac{\tau^{2}}{2}\right) M} \\
& = \begin{cases}\exp \left(\frac{2 D \Delta}{\tau B_{2}\left(1+p_{2,1)}\right.}+2 M\left(D+\frac{\Delta}{B_{2}\left(1+p_{2,1}\right)}\right)+\left(\tau+\frac{\tau^{2}}{2}\right) M\right) & \text { if } D \geq \frac{\Delta}{B_{2}\left(1+p_{2,1}\right)} \\
\exp \left(\frac{\left(D+\frac{\Delta}{B_{2}\left(1+p_{2,1}\right)}\right)^{2}}{2 \tau}+2 M\left(D+\frac{\Delta}{B_{2}\left(1+p_{2,1}\right)}\right)+\left(\tau+\frac{\tau^{2}}{2}\right) M\right) & \text { otherwise. }\end{cases}
\end{aligned}
$$

and using Equation (5.8),

$$
\begin{aligned}
\left(\epsilon^{\prime}(D, \Delta)\right)^{-2}=\exp \left[\left(B_{2} p_{2,1}^{2}\right.\right. & \left.+\frac{1}{2 \tau}\right)\left(\frac{\Delta}{B_{2}\left(1+p_{2,1}\right)}+D\right)^{2} \\
& \left.+\left(2 \Delta p_{2,1}+6 M\right)\left(\frac{\Delta}{B_{2}\left(1+p_{2,1}\right)}+D\right)+3 \tau\left(M+\frac{M^{2}}{2}\right)\right] .
\end{aligned}
$$

We note that the above expressions are nondecreasing functions of $D$. From Equation (4.21), we get, for $j=k+1, k+2$.

$$
D_{j} \leq C\left(\tau M+\mathcal{V}_{(k-1) \tau,(k+2) \tau}+2 \mathcal{W}_{(k-1) \tau,(k+2) \tau}\right) .
$$

The variables $\mathcal{V}_{(k-1) \tau,(k+2) \tau}$ and $\mathcal{W}_{(k-1) \tau,(k+2) \tau}$ are independent and can be controlled as in Equations $(4.34),(4.35)$. So we can bound

$$
\forall x \in \mathbb{R}, \mathbb{P}\left(\mathcal{V}_{(k-1) \tau,(k+2) \tau}+2 \mathcal{W}_{(k-1) \tau,(k+2) \tau} \geq x\right) \leq 2 \mathbb{P}\left(2 \mathcal{V}_{0,3 \tau} \geq \frac{x}{2}\right) \leq 4 \mathbb{P}\left(8\left|W_{3 \tau}\right| \geq x\right) .
$$

So, by Lemma 7.7,

$$
\begin{aligned}
& \mathbb{E}\left(\inf \left(1, \frac{T(\Delta)}{\left(\epsilon_{k+2}^{\prime}\right)^{4} \epsilon_{k+1}^{8}}\right)\right) \leq \int_{0}^{+\infty} \inf \{1, T(\Delta) \epsilon(C \tau M+C z, \Delta)^{-8} \\
&\left.\times \epsilon^{\prime}(C \tau M+C z, \Delta)^{-4}\right\} \frac{8 \exp \left(-\frac{z^{2}}{2(192 \tau)}\right)}{\sqrt{2 \pi \times 192 \tau}} d z .
\end{aligned}
$$

We have

$$
\int_{0}^{\left(\frac{\Delta}{C B_{2}\left(1+p_{2,1}\right.}-\tau M\right)}+{ }^{+} \epsilon(C \tau M+C z, \Delta)^{-8} \epsilon^{\prime}(C \tau M+C z, \Delta)^{-4} \frac{8 \exp \left(-\frac{z^{2}}{2(192 \tau)}\right)}{\sqrt{2 \pi \times 192 \tau}} d z
$$




$$
\begin{gathered}
\leq 8 \epsilon\left(\frac{\Delta}{B_{2}\left(1+p_{2,1}\right)}, \Delta\right)^{-8} \epsilon^{\prime}\left(\frac{\Delta}{B_{2}\left(1+p_{2,1}\right)}, \Delta\right)^{-4} \\
=8 \exp \left[\frac{20}{\tau}\left(\frac{\Delta}{B_{2}\left(1+p_{2,1}\right)}\right)^{2}+32\left(\frac{M \Delta}{B_{2}\left(1+p_{2,1}\right)}\right)+8\left(\tau+\frac{\tau^{2}}{2}\right) M+8 B_{2} p_{2,1}^{2}\left(\frac{\Delta}{B_{2}\left(1+p_{2,1}\right)}\right)^{2}\right. \\
\left.+8 \Delta p_{2,1}\left(\frac{\Delta}{B_{2}\left(1+p_{2,1}\right)}\right)+24 M\left(\frac{\Delta}{B_{2}\left(1+p_{2,1}\right)}\right)+6 \tau\left(M+\frac{M^{2}}{2}\right)\right] . \quad(6.12)
\end{gathered}
$$

From Subsection 2.5 (remember also Equation (4.3)), we get

$$
p_{2,1}=O\left(\frac{1}{\theta}\right), B_{2} \underset{\theta \rightarrow+\infty}{\longrightarrow} \frac{h}{2} .
$$

So there exists $\tau_{0}$, such that, for $\tau \geq \tau_{0}$,

$$
\begin{array}{r}
\log \left(\int_{0}^{\left(\frac{\Delta}{C B_{2}\left(1+p_{2,1}\right.}-\tau M\right)}{ }^{+} \inf \left\{1, T(\Delta) \epsilon(C \tau M+C z, \Delta)^{-8} \times \epsilon^{\prime}(C \tau M+C z, \Delta)^{-4}\right\}\right. \\
\left.\frac{8 \exp \left(-\frac{z^{2}}{2(192 \tau)}\right)}{\sqrt{2 \pi \times 192 \tau}} d z\right) \underset{\Delta, c}{\prec}-\inf \left(\frac{1}{h}, C_{0}\right) \Delta^{2} .
\end{array}
$$

We now want to bound

$$
\int_{\left(\frac{\Delta}{C B_{2}\left(1+p_{2,1}\right.}-\tau M\right)_{+}}^{+\infty} \inf \left\{1, T(\Delta) \epsilon(C \tau M+C z, \Delta)^{-8} \times \epsilon^{\prime}(C \tau M+C z, \Delta)^{-4}\right\} \frac{8 \exp \left(-\frac{z^{2}}{2(192 \tau)}\right)}{\sqrt{2 \pi \times 192 \tau}} d z .
$$

Let us set, for $z \geq\left(\frac{\Delta}{C B_{2}\left(1+p_{2,1}\right)}-\tau M\right)_{+}$,

$$
\begin{aligned}
& \Phi(\Delta, z)=T(\Delta) \epsilon(C \tau M+C z, \Delta)^{-8} \times \epsilon^{\prime}(C \tau M+C z, \Delta)^{-4} \\
& =T(\Delta) \times \exp \left(\frac{16(C \tau M+C z) \Delta}{\tau B_{2}\left(1+p_{2,1}\right)}+16 M\left(C \tau M+C z+\frac{\Delta}{B_{2}\left(1+p_{2,1}\right)}\right)+8\left(\tau+\frac{\tau^{2}}{2}\right) M\right) \\
& \quad \times \exp \left[\left(2 B_{2} p_{2,1}^{2}+\frac{1}{\tau}\right)\left(\frac{\Delta}{B_{2}\left(1+p_{2,1}\right)}+C \tau M+C z\right)^{2}\right. \\
& \left.\quad+\left(4 \Delta p_{2,1}+12 M\right)\left(\frac{\Delta}{B_{2}\left(1+p_{2,1}\right)}+C \tau M+C z\right)+6 \tau\left(M+\frac{M^{2}}{2}\right)\right] .
\end{aligned}
$$

For $z \leq\left(\frac{\Delta}{C B_{2}\left(1+p_{2,1}\right)}-\tau M\right)_{+}$, we define $\Phi(\Delta, z)$ by

$$
\begin{aligned}
& \Phi(\Delta, z)= \\
& T(\Delta) \times \exp \left(\frac{16(C \tau M+C z) \Delta}{\tau B_{2}\left(1+p_{2,1}\right)}+16 M\left(C \tau M+C z+\frac{\Delta}{B_{2}\left(1+p_{2,1}\right)}\right)+8\left(\tau+\frac{\tau^{2}}{2}\right) M\right) \\
& \times \exp \left[\left(2 B_{2} p_{2,1}^{2}+\frac{1}{\tau}\right)\left(\frac{\Delta}{B_{2}\left(1+p_{2,1}\right)}+C \tau M+C z\right)^{2}\right. \\
& \left.+\left(4 \Delta p_{2,1}+12 M\right)\left(\frac{\Delta}{B_{2}\left(1+p_{2,1}\right)}+C \tau M+C z\right)+6 \tau\left(M+\frac{M^{2}}{2}\right)\right] .
\end{aligned}
$$

For a constant $\bar{C}$ bigger than $C$, we define $\bar{\Phi}(\Delta, z)$ to have the same expression as $\Phi$, except that we replace $C$ by $\bar{C}$. We choose a

$$
\bar{C}=\sup \left(C, \frac{1}{\sqrt{2 \times 192}}\right)
$$


so that $z \mapsto \bar{\Phi}(\Delta, z) \times \exp \left(-z^{2} /(2 \times 192 \tau)\right)$ is nondecreasing in $z$. We have $\bar{\Phi}(\Delta, z) \rightarrow \infty$ when $z \rightarrow+\infty$ and $\Delta$ is fixed. Let us set, for a fixed $\Delta$,

$$
z_{0}=\inf \{z: \bar{\Phi}(\Delta, z) \geq 1\} .
$$

There exists $\lambda_{1}>0$ and $\tau_{1}>0$ such that for all $\tau \geq \tau_{1}$,

$$
T(\Delta) \epsilon\left(\bar{C} \tau M+\bar{C} \lambda_{1} \sqrt{\tau} \Delta, \Delta\right)^{-8} \epsilon^{\prime}\left(\bar{C} \tau M+\bar{C} \lambda_{1} \sqrt{\tau} \Delta, \Delta\right)^{-4} \underset{\Delta \rightarrow+\infty}{\longrightarrow} 0 .
$$

Looking at the definition of $p_{2,1}$ (Equation (4.3)) and at Equations (7.22), (7.20), we see that $\lambda_{1}$ can be chosen as a function of $h$, which we denote by $\lambda_{1}(h)$. And there exists $\lambda_{2}>0$ and $\tau_{2}>0$ such that for all $\tau \geq \tau_{2}$,

$$
T(\Delta) \epsilon\left(\bar{C} \tau M+\bar{C} \lambda_{2} \sqrt{\tau} \Delta, \Delta\right)^{-8} \epsilon^{\prime}\left(\bar{C} \tau M+\bar{C} \lambda_{2} \sqrt{\tau} \Delta, \Delta\right)^{-4} \underset{\Delta \rightarrow+\infty}{\longrightarrow}+\infty .
$$

So there exists $\Delta_{1}$ such that, for $\Delta$ bigger than $\Delta_{1}$ and $\tau$ bigger than $\sup \left(\tau_{1}, \tau_{2}\right)$,

$$
\lambda_{1} \sqrt{\tau} \Delta \leq z_{0} \leq \lambda_{2} \sqrt{\tau} \Delta .
$$

We can then bound, if $\tau \geq \sup \left(\tau_{1}, \tau_{2}\right)$,

$$
\begin{aligned}
\int_{0}^{z_{0}} \inf (1, \Phi(\Delta, z)) \frac{8 \exp \left(-\frac{z^{2}}{2(192 \tau)}\right)}{\sqrt{2 \pi(192 \tau)}} d z & \leq \int_{0}^{z_{0}} \inf (1, \bar{\Phi}(\Delta, z)) \frac{8 \exp \left(-\frac{z^{2}}{2(192 \tau)}\right)}{\sqrt{2 \pi(192 \tau)}} d z \\
& \leq z_{0} \bar{\Phi}\left(\Delta, z_{0}\right) \frac{8 \exp \left(-\frac{z_{0}^{2}}{2(192 \tau)}\right)}{\sqrt{2 \pi(192 \tau)}} \\
& =z_{0} \frac{8 \exp \left(-\frac{z_{0}^{2}}{2(192 \tau)}\right)}{\sqrt{2 \pi(192 \tau)}} \\
& \leq \lambda_{2} \sqrt{\tau} \Delta \frac{8 \exp \left(-\frac{\lambda_{1}^{2} \Delta^{2}}{2 \times 192}\right)}{\sqrt{2 \pi(192 \tau)}} .
\end{aligned}
$$

So, if $\tau \geq \sup \left(\tau_{0}, \tau_{1}, \tau_{2}\right)$, we get, using again Equation (4.36)

$$
\begin{gathered}
\int_{\left(\frac{\Delta}{C B_{2}\left(1+p_{2,1}\right)}-\tau^{2} M\right)_{+}}^{+\infty} \inf \left\{1, T(\Delta) \epsilon\left(C \tau^{2} M+C z, \Delta\right)^{-8} \times \epsilon^{\prime}\left(C \tau^{2} M+C z, \Delta\right)^{-4}\right\} \\
\quad \times \frac{8 \exp \left(-\frac{z^{2}}{2(192 \tau)}\right)}{\sqrt{2 \pi \times 192 \tau}} d z \\
\preceq \int_{0}^{z_{0}} \Phi(\Delta, z) \frac{e^{-\frac{z^{2}}{2(192 \tau)}}}{\sqrt{2 \pi \times 192 \tau}} d z+\int_{z_{0}}^{+\infty} \frac{e^{-\frac{z^{2}}{2(192 \tau)}}}{\sqrt{2 \pi \times 192 \tau}} d z \\
\leq \lambda_{2} \sqrt{\tau} \Delta \frac{8 \exp \left(-\frac{\lambda_{1}^{2} \Delta^{2}}{384}\right)}{\sqrt{2 \pi(192 \tau)}}+\frac{e^{-\frac{z_{0}^{2}}{2(192 \tau)}}}{z_{0} \sqrt{2 \pi}} \times \sqrt{192 \tau}
\end{gathered}
$$

and so

$$
\begin{array}{r}
\log \left(\int_{-\infty}^{+\infty} \inf \left(1, T(\Delta) \epsilon(C \tau M+C z, \Delta)^{-8} \times \epsilon^{\prime}(C \tau M+C z, \Delta)^{-4}\right) \frac{e^{-\frac{z^{2}}{2(192 \tau)}}}{\sqrt{2 \pi \times 192 \tau}} d z\right) \\
\preceq-\Delta^{2}-\Delta^{2} \inf \left(\frac{1}{h}, C_{0}, \lambda_{1}^{2}\right) .
\end{array}
$$


In the remaining of the proof, we will suppose $\tau \geq \sup \left(\tau_{0}, \tau_{1}, \tau_{2}\right)$. Looking at Equation (6.2), we see that we can now bound all the terms on its right-hand side. We have

$$
\mathbb{E}\left(\left\|\pi_{n \tau}-\bar{R}_{n}^{\Delta}\left(\pi_{(n-1) \tau}\right)\right\|\right) \preceq T(\Delta),
$$

by Proposition 4.7. Recall, that, from Equations (6.7), (4.22), we get

$$
\log (T(\Delta)) \underset{\Delta, c}{\preceq}-\Delta^{2} \inf \left(\frac{1}{h}, C_{0}\right) .
$$

For $k$ in $\{1, \ldots, n-1\}$, we have bounded

$$
\begin{aligned}
& \mathbb{E}\left(\| \bar{R}_{n: k+1}^{\Delta}\left(\pi_{k \Delta}\right)-\bar{R}_{n: k+1}^{\Delta}\left(\bar{R}_{k}^{\Delta}\left(\pi_{(k-1) \Delta}\right) \|\right)\right. \\
& \quad \leq\left(1-\frac{\epsilon(L, \Delta)^{2} \epsilon^{\prime}(L, \Delta)^{2}}{2}\right)^{\left\lceil\frac{1}{2}\left(\left\lfloor\frac{n-k}{2}\right\rfloor-4\right)_{+}\right\rceil} \times 2 \mathbb{E}\left(\inf \left(1, \frac{\left\|\pi_{k \tau}-\bar{R}_{k}^{\Delta}\left(\pi_{(k-1) \tau}\right)\right\|}{\left(\epsilon_{k+2}^{\prime}\right)^{2} \epsilon_{k+1}^{4}}\right)\right) .
\end{aligned}
$$

And the last expectation can be bounded by the sum of the following expectations :

$$
\begin{gathered}
\mathbb{E}\left(\inf \left(1, \frac{\left\|\pi_{k \tau}-\bar{R}_{k}^{\Delta}\left(\pi_{(k-1) \tau}\right)\right\|^{2}}{T(\Delta)}\right)\right) \preceq \sqrt{T(\Delta)}, \\
\mathbb{E}\left(\inf \left(1, \frac{T(\Delta)}{\left(\epsilon_{k+2}^{\prime}\right)^{4} \epsilon_{k+1}^{8}}\right)\right) \leq \exp \left(-\widehat{B}_{1} \Delta^{2} \inf \left(\frac{1}{h}, C_{0}, \lambda_{1}^{2}\right)\right) \text { for } \Delta \geq \Delta_{0}(\tau),
\end{gathered}
$$

for some constant $\widehat{B}_{1}$ and some function $\Delta_{0}$, where the bounds come from Equations (6.6), (6.11), (6.17) (we also use Lemma 2.6). The constant $\widehat{B}_{1}$ above is universal and $\Delta_{0}$ is continuous in $\tau$. So we get, for all $\Delta \geq \Delta_{0}(\tau)$, using Equation (6.18),

$$
\mathbb{E}\left(\left\|\pi_{n \tau}-\pi_{n \tau}^{\Delta}\right\|\right) \leq \exp \left(-\widehat{C}_{1} \Delta^{2} \inf \left(\frac{1}{h}, C_{0}, \lambda_{1}^{2}\right)\right) \sum_{k \geq 0}\left(1-\frac{\epsilon(L, \Delta)^{4} \epsilon^{\prime}(L, \Delta)^{2}}{2}\right)^{\left\lceil\frac{1}{2}\left(\left\lfloor\frac{n-k}{2}\right\rfloor-4\right)_{+}\right\rceil},
$$

(for some universal constant $\widehat{C}_{1}$ ) from which we get

$$
\sup _{n \geq 0} \log \mathbb{E}\left(\left\|\pi_{n \tau}-\pi_{n \tau}^{\Delta}\right\|\right) \underset{\Delta, c}{\preceq}-\log \left(\epsilon(L, \Delta) \epsilon^{\prime}(L, \Delta)\right)-\Delta^{2} \inf \left(\frac{1}{h}, C_{0}, \lambda_{1}^{2}\right) .
$$

Looking at Equations (6.8), (6.9), we see there exists $\tau_{3}$, such that, for $\tau>\tau_{3}$,

$$
\sup _{n \geq 0} \log \mathbb{E}\left(\left\|\pi_{n \tau}-\pi_{n \tau}^{\Delta}\right\|\right) \underset{\Delta, c}{\preceq}-\Delta^{2} \inf \left(\frac{1}{h}, C_{0}, \lambda_{1}^{2}\right) .
$$

\subsection{Stability of the optimal filter}

Proof of Theorem 1.2. We decompose, for all $n$,

$$
\begin{aligned}
\left\|\pi_{n \tau}-\pi_{n \tau}^{\prime}\right\| \leq\left\|\pi_{n \tau}-\bar{R}_{n}^{\Delta} \ldots \bar{R}_{1}^{\Delta}\left(\pi_{0}\right)\right\|+\left\|\bar{R}_{n}^{\Delta} \ldots \bar{R}_{1}^{\Delta}\left(\pi_{0}\right)-\bar{R}_{n}^{\Delta} \ldots \bar{R}_{1}^{\Delta}\left(\pi_{0}^{\prime}\right)\right\| & \\
& +\left\|\bar{R}_{n}^{\Delta} \ldots \bar{R}_{1}^{\Delta}\left(\pi_{0}\right)-\pi_{n \tau}^{\prime}\right\| .
\end{aligned}
$$

Let $\tau_{\infty}$ be the parameter defined in Proposition 1.5. Recall that the operators $\left(R_{n}\right)_{n \geq 0},\left(R_{n}^{\Delta}\right)_{n \geq 0}$ depend on $\tau$. Suppose that $L$ is such that (as in Equation (6.1))

$$
L>3\left|m_{0}\right|+3 C M \tau_{\infty}, \widetilde{\alpha}(L) \leq \frac{1}{4} .
$$


Then, as in Equation (6.4), we have, for all $\tau \in\left[\tau_{\infty}, 2 \tau_{\infty}\right]$, for all $n \geq 0$,

$$
\begin{aligned}
\mathbb{E}\left(\left\|\bar{R}_{n}^{\Delta} \ldots \bar{R}_{1}^{\Delta}\left(\pi_{0}\right)-\bar{R}_{n}^{\Delta} \ldots \bar{R}_{1}^{\Delta}\left(\pi_{0}^{\prime}\right)\right\|\right) & \\
\leq\left(1-\frac{\epsilon(L, \Delta)^{2} \epsilon^{\prime}(L, \Delta)^{2}}{2}\right)^{\left\lceil\frac{1}{2}\left(\left\lfloor\frac{n}{2}\right\rfloor-4\right)_{+}\right\rceil} & \times 2 \mathbb{E}\left(\inf \left(1, \frac{\left\|\pi_{0}-\pi_{0}^{\prime}\right\|}{\left(\epsilon_{2}^{\prime}\right)^{2} \epsilon_{1}^{4}}\right)\right) \\
& \leq 2\left(1-\frac{\epsilon(L, \Delta)^{2} \epsilon^{\prime}(L, \Delta)^{2}}{2}\right)^{\left\lceil\frac{1}{2}\left(\left\lfloor\frac{n}{2}\right\rfloor-4\right)_{+}\right\rceil} .
\end{aligned}
$$

We have (using Equations (6.8), (6.9))

$$
\log \left(\epsilon(L, \Delta) \epsilon^{\prime}(L, \Delta)\right) \underset{\Delta, c}{\succeq}-\left(\frac{\Delta^{2}}{\tau B_{2}^{2}\left(1+p_{2,1}\right)^{2}}+\frac{\left(B_{2} p_{2,1}^{2}+\frac{1}{\tau}\right) \Delta^{2}}{B_{2}^{2}\left(1+p_{2,1}\right)^{2}}+\frac{p_{2,1} \Delta^{2}}{B_{2}\left(1+p_{2,1}\right)}\right) .
$$

We now take a sequence $\Delta_{n}=\sqrt{\nu \log (n)}$, for some $\nu>0$. By Lemma 2.6, 3, there exist a constants $b_{1}$ and an integer $n_{0}$ such that, for all $\tau \in\left[\tau_{\infty}, 2 \tau_{\infty}\right]$, for $n>n_{0}$,

$$
\begin{aligned}
& \left(1-\frac{\left(\epsilon\left(L, \Delta_{n}\right) \epsilon^{\prime}\left(L, \Delta_{n}\right)\right)^{2}}{2}\right)^{\left\lceil\frac{1}{2}\left(\left\lfloor\frac{n}{2}\right\rfloor-4\right)_{+}\right\rceil} \\
& \quad \leq \exp \left[-\frac{1}{2}\left[\frac{1}{2}\left(\left\lfloor\frac{n}{2}\right\rfloor-4\right)_{+}\right]\right. \\
& \left.\quad \times \exp \left(-b_{1} \Delta_{n}^{2}\left(\frac{1}{\tau B_{2}^{2}\left(1+p_{2,1}\right)^{2}}+\frac{B_{2} p_{2,1}^{2}+\frac{1}{\tau}}{B_{2}^{2}\left(1+p_{2,1}\right)^{2}}+\frac{p_{2,1}}{B_{2}\left(1+p_{2,1}\right)}\right)\right)\right] \\
& \quad=\exp \left[-\frac{1}{2}\left[\frac{1}{2}\left(\left\lfloor\frac{n}{2}\right\rfloor-4\right)_{+}\right] n^{-\nu^{\prime}}\right],
\end{aligned}
$$

with

$$
\nu^{\prime}=b_{1} \nu\left(\frac{1}{\tau B_{2}^{2}\left(1+p_{2,1}\right)^{2}}+\frac{B_{2} p_{2,1}^{2}+\frac{1}{\tau}}{B_{2}^{2}\left(1+p_{2,1}\right)^{2}}+\frac{p_{2,1}}{B_{2}\left(1+p_{2,1}\right)}\right) .
$$

By Proposition 1.5, we know there exists a constants $b_{1}^{\prime}$ and a integer $n_{0}^{\prime}$ such that, for all $\tau \in$ $\left[\tau_{\infty}, 2 \tau_{\infty}\right]$ and $n \geq n_{0}^{\prime}$,

$$
\begin{array}{r}
\sup \left(\mathbb{E}\left(\left\|\pi_{n \tau}-\bar{R}_{n}^{\Delta_{n}} \ldots \bar{R}_{1}^{\Delta_{n}}\left(\pi_{0}\right)\right\|\right), \mathbb{E}\left(\left\|\pi_{n \tau}^{\prime}-\bar{R}_{n}^{\Delta_{n}} \ldots \bar{R}_{1}^{\Delta_{n}}\left(\pi_{0}^{\prime}\right)\right\|\right)\right) \\
\quad \leq \exp \left(-b_{1}^{\prime} \Delta_{n}^{2} \lambda_{1}^{\prime}(h)\right) \leq n^{-\nu^{\prime \prime}}
\end{array}
$$

with $\nu^{\prime \prime}=b_{1}^{\prime} \nu \lambda_{1}^{\prime}(h)$. Let us set $\epsilon \in(0,1)$. We choose

$$
\nu=\frac{(1-\epsilon)}{b_{1}}\left(\frac{1}{\tau B_{2}^{2}\left(1+p_{2,1}\right)^{2}}+\frac{B_{2} p_{2,1}^{2}+\frac{1}{\tau}}{B_{2}^{2}\left(1+p_{2,1}\right)^{2}}+\frac{p_{2,1}}{B_{2}\left(1+p_{2,1}\right)}\right)^{-1},
$$

which leads to $\nu^{\prime}=1-\epsilon$. We set $\nu_{0}=\nu^{\prime \prime}$. For any $t \geq \tau_{\infty}$, if we set $n=\left\lfloor t / \tau_{\infty}\right\rfloor$, then $t=n \tau$ with $\tau \in\left[\tau_{\infty}, 2 \tau_{\infty}\right]$, and so :

$$
\begin{gathered}
\mathbb{E}\left(\left\|\pi_{t}-\pi_{t}^{\prime}\right\|\right) \leq 2 n^{-\nu_{0}}+2 \exp \left(-\frac{1}{2}\left\lceil\frac{1}{2}\left(\left\lfloor\frac{n-k}{2}\right\rfloor-4\right)_{+}\right\rceil n^{-\nu^{\prime}}\right), \\
\mathbb{E}\left(\left\|\pi_{t}-\pi_{t}^{\prime}\right\|\right)=O\left(t^{-\nu_{0}}\right) .
\end{gathered}
$$

Remark 6.2. One could seek to obtain a sharper bound in the above Theorem by choosing another sequence $\left(\Delta_{n}\right)_{n \geq 0}$. Up to some logarithmic terms, the bound would still be a power of $t$. 


\section{Appendix}

\subsection{Proofs of Section 1}

Proof of Lemma 2.1. Following [BC09] (Chapter 6, Section 6.1), we introduce the process

$$
\widehat{V}_{t}=V_{t}+\int_{0}^{t} f\left(X_{s}\right) d s, t \geq 0 .
$$

We introduce a new probability $\widetilde{\mathbb{P}}$ defined by

$$
\left.\frac{d \mathbb{P}}{d \widetilde{\mathbb{P}}}\right|_{\mathcal{F}_{t}}=\exp \left(\int_{0}^{t} f\left(X_{s}\right) d \widehat{V}_{s}-\frac{1}{2} \int_{0}^{t} f\left(X_{s}\right)^{2} d s\right) .
$$

By Girsanov's theorem, $\widehat{V}$ is a standard Brownian motion under $\widetilde{\mathbb{P}}$. We set $F$ to be a primitive of $f$. We have, for all $t \geq 0$,

$$
\begin{aligned}
\int_{0}^{t} f\left(X_{s}\right) d \widehat{V}_{s}-\frac{1}{2} \int_{0}^{t} f\left(X_{s}\right)^{2} d s & =\int_{0}^{t} f\left(X_{s}\right) d X_{s}-\frac{1}{2} \int_{0}^{t} f\left(X_{s}\right)^{2} d s \\
& =F\left(X_{t}\right)-F\left(X_{0}\right)-\frac{1}{2} \int_{0}^{t} f^{\prime}\left(X_{s}\right) d s-\frac{1}{2} \int_{0}^{t} f\left(X_{s}\right)^{2} d s \\
& \geq-M\left|X_{t}-X_{0}\right|-\frac{M t}{2}-\frac{M^{2} t}{2} .
\end{aligned}
$$

So, for any test function $\varphi$ in $\mathcal{C}_{b}^{+}(\mathbb{R})$ (the set of bounded continuous functions on $\mathbb{R}$ ), $t \geq 0$

$$
\begin{aligned}
\mathbb{E}\left(\varphi\left(X_{t}\right)\right) & =\mathbb{E}^{\mathbb{P}}\left(\varphi\left(X_{t}\right)\right) \\
& =\mathbb{E}^{\widetilde{\mathbb{P}}}\left(\left.\varphi\left(X_{t}\right) \frac{d \mathbb{P}}{d \widetilde{\mathbb{P}}}\right|_{\mathcal{F}_{t}}\right) \\
& \geq \mathbb{E}^{\widetilde{\mathbb{P}}}\left(\varphi\left(X_{t}\right) \exp \left(-M\left|X_{t}-X_{0}\right|-\frac{M t}{2}-\frac{M^{2} t}{2}\right)\right) .
\end{aligned}
$$

Similarly:

$$
\mathbb{E}\left(\varphi\left(X_{t}\right)\right) \leq \mathbb{E}^{\widetilde{\mathbb{P}}}\left(\varphi\left(X_{t}\right) \exp \left(M\left|X_{t}-X_{0}\right|+\frac{M t}{2}\right)\right)
$$

So we have the result.

Proof of Lemma 2.2. For any test function $\varphi$ in $\mathcal{C}_{b}^{+}([0, t])$ and any $t \geq 0$,

$$
\begin{aligned}
\mathbb{E}^{\mathbb{P}}\left(\varphi\left(Y_{0: t}\right) \mid X_{0}, X_{t}\right) & =\frac{\mathbb{E}^{\widehat{\mathbb{P}}}\left(\left.\varphi\left(Y_{0: t}\right) \frac{d \mathbb{P}}{d \mathbb{P}}\right|_{\mathcal{F}_{t}} \mid X_{0}, X_{t}\right)}{\mathbb{E}^{\widehat{\mathbb{P}}}\left(\left.\frac{d \mathbb{P}}{d \widehat{\mathbb{P}}}\right|_{\mathcal{F}_{t}} \mid X_{0}, X_{t}\right)} \\
& =\frac{\mathbb{E}^{\widehat{\mathbb{P}}}\left(\varphi\left(Y_{0: t}\right) \mathbb{E}^{\widehat{\mathbb{P}}}\left(\left.\frac{d \mathbb{P}}{d \mathbb{P}}\right|_{\mathcal{F}_{t}} \mid X_{0}, X_{t}, Y_{0: t}\right) \mid X_{0}, X_{t}\right)}{\mathbb{E}^{\widehat{\mathbb{P}}}\left(\left.\frac{d \mathbb{P}}{d \widehat{\mathbb{P}}}\right|_{\mathcal{F}_{t}} \mid X_{0}, X_{t}\right)} .
\end{aligned}
$$

By Girsanov's Theorem, $(\widehat{V}, Y)$ is a standard two-dimensional Brownian motion under $\widehat{\mathbb{P}}$. So, conditionally on $X_{0}, X_{t}$, the law of $Y_{0: t}$ under $\mathbb{P}$ has the following density with respect to the Wiener measure:

$$
y_{0: t} \mapsto \psi_{t}\left(Y_{0: t}, X_{0}, X_{t}\right)=\frac{\mathbb{E}^{\widehat{\mathbb{P}}}\left(\left.\frac{d \mathbb{P}}{d \widehat{\mathbb{P}}}\right|_{\mathcal{F}_{t}} \mid X_{0}, X_{t}, Y_{0: t}\right)}{\mathbb{E}^{\widehat{\mathbb{P}}}\left(\left.\frac{d \mathbb{P}}{d \widehat{\mathbb{P}}}\right|_{\mathcal{F}_{t}} \mid X_{0}, X_{t}\right)}
$$


We have

$$
\begin{aligned}
\left.\frac{d \mathbb{P}}{d \widehat{\mathbb{P}}}\right|_{\mathcal{F}_{t}}=\exp \left(F\left(X_{1}\right)-F\left(X_{0}\right)\right. & -\frac{1}{2} \int_{0}^{t} f^{\prime}\left(X_{s}\right) d s-\frac{1}{2} \int_{0}^{1} f\left(X_{s}\right)^{2} d s \\
& \left.+\int_{0}^{t} h X_{s} d Y_{s}-\frac{1}{2} \int_{0}^{t} h^{2} X_{s}^{2} d s\right) \\
& \mathbb{E}^{\widehat{\mathbb{P}}}\left(\left.\frac{d \mathbb{P}}{d \widehat{\mathbb{P}}}\right|_{\mathcal{F}_{t}} \mid X_{0}, X_{t}\right)=\mathbb{E}^{\widehat{\mathbb{P}}}\left(\exp \left(F\left(X_{1}\right)-F\left(X_{0}\right)-\frac{1}{2} \int_{0}^{t} f^{\prime}\left(X_{s}\right) d s-\frac{1}{2} \int_{0}^{t} f\left(X_{s}\right)^{2} d s\right) \mid X_{0}, X_{1}\right),
\end{aligned}
$$

so

$$
\exp \left(-M\left|X_{t}-X_{0}\right|-\frac{t\left(M+M^{2}\right)}{2}\right) \leq \mathbb{E}^{\widehat{\mathbb{P}}}\left(\left.\left.\frac{d \mathbb{P}}{d \widehat{\mathbb{P}}}\right|_{\mathcal{F}_{t}}\right|_{0}, X_{t}\right) \leq \exp \left(M\left|X_{t}-X_{0}\right|+\frac{t M}{2}\right) .
$$

Using the above calculations (Equation (7.2)), we can write:

$$
\begin{aligned}
\exp \left(-M\left|X_{t}-X_{0}\right|-\frac{t\left(M+M^{2}\right)}{2}\right) \widehat{\psi}_{t}\left(Y_{0: t}, x_{0}, x_{1}\right) \leq \\
\mathbb{E}^{\widehat{\mathbb{P}}}\left(\left.\frac{d \mathbb{P}}{d \widehat{\mathbb{P}}}\right|_{\mathcal{F}_{t}} \mid X_{0}, X_{t}, Y_{0: t}\right) \leq \exp \left(M\left|X_{t}-X_{0}\right|+\frac{t M}{2}\right) \widehat{\psi}\left(Y_{0: t}, x_{0}, x_{1}\right) .
\end{aligned}
$$

So we have the result.

Proof of Lemma 1.1. We define a new probability $\check{\mathbb{P}}$ by

$$
\left.\frac{d \mathbb{P}}{d \check{\mathbb{P}}}\right|_{\mathcal{F}_{t}}=\exp \left(\int_{0}^{t} h X_{s} d Y_{s}-\frac{1}{2} \int_{0}^{t} h^{2} X_{s}^{2} d s\right), \forall t \geq 0 .
$$

By Girsanov's Theorem, $\left(Y_{t}\right)$ is a Brownian motion under $\check{\mathbb{P}}$. For all bounded continuous $\varphi$ and all $t \geq 0$, we have (Kallianpur-Striebel, see [BC09], p.57)

$$
\mathbb{E}\left(\varphi\left(X_{t}\right) \mid Y_{0: t}\right)=\frac{\mathbb{E}^{\check{\mathbb{P}}}\left(\left.\varphi\left(X_{t}\right) \frac{d \mathbb{P}}{d \mathbb{P}}\right|_{\mathcal{F}_{t}} \mid Y_{0: t}\right)}{\mathbb{E}^{\check{\mathbb{P}}}\left(\frac{d \mathbb{P}}{d \mathbb{P}} \mid Y_{0: t}\right)}
$$

and

$$
\mathbb{E}^{\check{\mathbb{P}}}\left(\left.\varphi\left(X_{t}\right) \frac{d \mathbb{P}}{d \check{\mathbb{P}}}\right|_{\mathcal{F}_{t}} \mid Y_{0: t}\right)=\mathbb{E}^{\check{\mathbb{P}}}\left(\varphi\left(X_{t}\right) \mathbb{E}^{\check{\mathbb{P}}}\left(\left.\frac{d \mathbb{P}}{d \check{\mathbb{P}}}\right|_{\mathcal{F}_{t}} \mid Y_{0: t}, X_{0}, X_{t}\right) \mid Y_{0: t}\right)
$$

and

$$
\begin{aligned}
& \mathbb{E}^{\check{\mathbb{P}}}\left(\left.\frac{d \mathbb{P}}{d \check{\mathbb{P}}}\right|_{\mathcal{F}_{t}} \mid Y_{0: t}, X_{0}, X_{t}\right)=\frac{\mathbb{E}^{\widehat{\mathbb{P}}}\left(\left.\frac{d \mathbb{\mathbb { P }}}{d \mathbb{P}}\right|_{\mathcal{F}_{t}} \frac{d \check{\mathbb{P}}}{\left.d\right|_{\mathcal{F}}} \mid Y_{0: t}, X_{0}, X_{t}\right)}{\mathbb{E}^{\widehat{\mathbb{P}}}\left(\left.\frac{d \tilde{\mathbb{P}}}{d \mathbb{P}}\right|_{\mathcal{F}_{t}} \mid Y_{0: t}, X_{0}, X_{t}\right)} \\
& =\frac{\psi_{t}\left(Y_{0: t}, X_{0}, X_{t}\right) \mathbb{E}^{\widehat{\mathbb{P}}}\left(\left.\frac{d \mathbb{P}}{d \widehat{\mathbb{P}}}\right|_{\mathcal{F}_{t}} \mid X_{0}, X_{t}\right)}{\mathbb{E}^{\widehat{\mathbb{P}}}\left(\left.\frac{d \mathbb{P}}{d \widetilde{\mathbb{P}}}\right|_{\mathcal{F}_{t}} \mid Y_{0: t}, X_{0}, X_{t}\right)} \\
& =\psi_{t}\left(Y_{0: t}, X_{0}, X_{t}\right) \times \frac{\mathbb{E}^{\widehat{\mathbb{P}}}\left(\left.\frac{d \mathbb{P}}{d \mathbb{P}}\right|_{\mathcal{F}_{t}} \mid X_{0}, X_{t}\right)}{\mathbb{E}^{\widehat{\mathbb{P}}}\left(\left.\frac{d \mathbb{P}}{d \mathbb{P}}\right|_{\mathcal{F}_{t}} \mid X_{0}, X_{t}\right)}
\end{aligned}
$$




$$
=\psi_{t}\left(Y_{0: t}, X_{0}, X_{t}\right) .
$$

As the law of $\left(X_{s}\right)_{s \geq 0}$ is the same under $\mathbb{P}$ or $\check{\mathbb{P}}$, we get the desired result.

\subsection{Proofs of Section 2}

We first prove two technical Lemmas.

Lemma 7.1. For any $t>0$, for any function $g: \mathbb{R} \mapsto \mathbb{R}$ which is measurable with respect to the Lebesgue measure and such that $\int_{0}^{t} g(s)^{2} d s<\infty$, we have :

$$
\int_{0}^{t} g(s) d B_{s}=\int_{0}^{t}\left(g(s)-\theta e^{\theta s} \int_{s}^{t} e^{-\theta u} g(u) d u\right) d \beta_{s} .
$$

Proof. Under $\mathbb{Q}, B$ is an Ornstein-Uhlenbeck process (see Equation (2.2)). We can write $B$ as the strong solution of (2.2):

$$
B_{t}=e^{-\theta t} \int_{0}^{t} e^{\theta s} d \beta_{s}, \forall t \geq 0 .
$$

We use the integration by parts formula to compute:

$$
\begin{gathered}
\int_{0}^{t}\left(g(s)-\theta e^{\theta s} \int_{s}^{t} e^{-\theta u} g(u) d u\right) d \beta_{s} \\
=\int_{0}^{t}\left(g(s)-\theta e^{\theta s} \int_{0}^{t} e^{-\theta u} g(u) d u\right) d \beta_{s}+\int_{0}^{t}\left(\theta e^{\theta s} \int_{0}^{s} e^{-\theta u} g(u) d u\right) d \beta_{s} \\
=\int_{0}^{t} g(s) d \beta_{s}-\left(\int_{0}^{t} e^{-\theta u} g(u) d u\right)\left(\int_{0}^{t} \theta e^{\theta s} d \beta_{s}\right)+\int_{0}^{t}\left(\theta e^{\theta s} \int_{0}^{s} e^{-\theta u} g(u) d u\right) d \beta_{s} \\
=\int_{0}^{t} g(s) d \beta_{s}-\int_{0}^{t} e^{-\theta u} g(u)\left(\int_{0}^{u} \theta e^{\theta s} d \beta_{s}\right) d u-\int_{0}^{t}\left(\int_{0}^{s} e^{-\theta u} g(u) d u\right) \theta e^{\theta s} d \beta_{s} \\
+\int_{0}^{t}\left(\theta e^{\theta s} \int_{0}^{s} e^{-\theta u} g(u) d u\right) d \beta_{s} \\
=\int_{0}^{t} g(s) d \beta_{s}-\int_{0}^{t} \theta g(u) B_{u} d u=\int_{0}^{t} g(s) d B_{s} .
\end{gathered}
$$

Lemma 7.2. We have, for all $s, t \geq 0$,

$$
g(s)-\theta e^{\theta s} \int_{s}^{t} e^{-\theta u} g(u) d u= \begin{cases}e^{\theta(s-t)} & \text { if } g(u)=1, \forall u, \\ \left(t+\frac{1}{\theta}\right) e^{\theta(s-t)}-\frac{1}{\theta} & \text { if } g(u)=u, \forall u, \\ \left(t^{2}+\frac{2 t}{\theta}+\frac{2}{\theta^{2}}\right) e^{\theta(s-t)}-\left(\frac{2 s}{\theta}+\frac{2}{\theta^{2}}\right) & \text { if } g(u)=u^{2}, \forall u\end{cases}
$$

Proof. The proof in the case $g(u)=1$ is straightforward. We compute, for all $s, t \geq 0$ :

$$
\begin{aligned}
s-\theta e^{\theta s} \int_{s}^{t} u e^{-\theta u} d u & =s-\theta e^{\theta s}\left[\left(-\frac{u}{\theta}-\frac{1}{\theta^{2}}\right) e^{-\theta u}\right]_{s}^{t} \\
& =s-\theta\left(-\frac{t}{\theta}-\frac{1}{\theta^{2}}\right) e^{\theta(s-t)}+\theta\left(-\frac{s}{\theta}-\frac{1}{\theta^{2}}\right) \\
& =\left(t+\frac{1}{\theta}\right) e^{\theta(s-t)}-\frac{1}{\theta} \\
s^{2}-\theta e^{\theta s} \int_{s}^{t} u^{2} e^{-\theta u} d u & =s^{2}-\theta e^{\theta s}\left[\left(-\frac{u^{2}}{\theta}-\frac{2 u}{\theta^{2}}-\frac{2}{\theta^{3}}\right) e^{-\theta u}\right]_{s}^{t}
\end{aligned}
$$




$$
\begin{aligned}
& =s^{2}-\theta e^{\theta s}\left(-\frac{t^{2}}{\theta}-\frac{2 t}{\theta^{2}}-\frac{2}{\theta^{3}}\right) e^{-\theta t}+\theta e^{\theta s}\left(-\frac{s^{2}}{\theta}-\frac{2 s}{\theta^{2}}-\frac{2}{\theta^{3}}\right) e^{-\theta s} \\
& =\left(t^{2}+\frac{2 t}{\theta}+\frac{2}{\theta^{2}}\right) e^{\theta(s-t)}-\left(\frac{2 s}{\theta}+\frac{2}{\theta^{2}}\right) .
\end{aligned}
$$

Proof of Lemma 2.3. Lemma 7.1 tells us that the variables $G_{1}, G_{2}, G_{3}, G_{4}$ are centered Gaussians under $\mathbb{Q}$. Using Lemma 7.2, we compute the following expectations:

$$
\begin{aligned}
& \mathbb{E}^{\mathbb{Q}}\left(G_{1}^{2}\right)=\int_{0}^{1} e^{2 \theta(s-1)} d s \\
& =\frac{1-e^{-2 \theta}}{2 \theta}, \\
& \mathbb{E}^{\mathbb{Q}}\left(G_{2}^{2}\right)=\int_{0}^{1}\left(\left(1+\frac{1}{\theta}\right) e^{\theta(s-1)}-\frac{1}{\theta}\right)^{2} d s \\
& =\int_{0}^{1}\left(1+\frac{1}{\theta}\right)^{2} e^{2 \theta(s-1)}+\frac{1}{\theta^{2}}-\frac{2}{\theta}\left(1+\frac{1}{\theta}\right) e^{\theta(s-1)} d s \\
& =\left[\left(1+\frac{1}{\theta}\right)^{2} \frac{e^{2 \theta(s-1)}}{2 \theta}+\frac{s}{\theta^{2}}-\left(1+\frac{1}{\theta}\right) \frac{2 e^{\theta(s-1)}}{\theta^{2}}\right]_{0}^{1} \\
& =\left(1+\frac{1}{\theta}\right)^{2} \frac{\left(1-e^{-2 \theta}\right)}{2 \theta}+\frac{1}{\theta^{2}}-\left(\frac{2}{\theta^{2}}+\frac{2}{\theta^{3}}\right)\left(1-e^{-\theta}\right) \text {, } \\
& \mathbb{E}^{\mathbb{Q}}\left(G_{3}^{2}\right)=\int_{0}^{1}\left(\left(1+\frac{2}{\theta}+\frac{2}{\theta^{2}}\right) e^{\theta(s-1)}-\left(\frac{2 s}{\theta}+\frac{2}{\theta^{2}}\right)\right)^{2} d s= \\
& \int_{0}^{1}\left(1+\frac{2}{\theta}+\frac{2}{\theta^{2}}\right)^{2} e^{2 \theta(s-1)}+\left(\frac{2 s}{\theta}+\frac{2}{\theta^{2}}\right)^{2}-2\left(1+\frac{2}{\theta}+\frac{2}{\theta^{2}}\right) e^{\theta(s-1)}\left(\frac{2 s}{\theta}+\frac{2}{\theta^{2}}\right) d s= \\
& {\left[\left(1+\frac{2}{\theta}+\frac{2}{\theta^{2}}\right)^{2} \frac{e^{2 \theta(s-1)}}{2 \theta}+\left(\frac{2 s}{\theta}+\frac{2}{\theta^{2}}\right)^{3} \frac{\theta}{6}-2\left(1+\frac{2}{\theta}+\frac{2}{\theta^{2}}\right)\left(\frac{2 s}{\theta^{2}}\right) e^{\theta(s-1)}\right]_{0}^{1}=} \\
& \left(1+\frac{2}{\theta}+\frac{2}{\theta^{2}}\right)^{2} \frac{\left(1-e^{-2 \theta}\right)}{2 \theta}+\left(\frac{2}{\theta}+\frac{2}{\theta^{2}}\right)^{3} \frac{\theta}{6}-\frac{8}{6 \theta^{5}}-\frac{4}{\theta^{2}}\left(1+\frac{2}{\theta}+\frac{2}{\theta^{2}}\right), \\
& \mathbb{E}^{\mathbb{Q}}\left(G_{1} G_{2}\right)=\int_{0}^{1} e^{\theta(s-1)} \times\left(\left(1+\frac{1}{\theta}\right) e^{\theta(s-1)}-\frac{1}{\theta}\right) d s \\
& =\left[\left(1+\frac{1}{\theta}\right) \frac{e^{2 \theta(s-1)}}{2 \theta}-\frac{e^{\theta(s-1)}}{\theta^{2}}\right]_{0}^{1}=\left(\frac{1}{2 \theta}+\frac{1}{2 \theta^{2}}\right)\left(1-e^{-2 \theta}\right)+\frac{e^{-\theta}-1}{\theta^{2}}, \\
& \mathbb{E}^{\mathbb{Q}}\left(G_{1} G_{3}\right)=\int_{0}^{1} e^{\theta(s-1)}\left(\left(1+\frac{2}{\theta}+\frac{2}{\theta^{2}}\right) e^{\theta(s-1)}-\left(\frac{2 s}{\theta}+\frac{2}{\theta^{2}}\right)\right) d s \\
& =\left[\left(1+\frac{2}{\theta}+\frac{2}{\theta^{2}}\right) \frac{e^{2 \theta(s-1)}}{2 \theta}-\frac{2 s}{\theta^{2}} e^{\theta(s-1)}\right]_{0}^{1}=\left(\frac{1}{2 \theta}+\frac{1}{\theta^{2}}+\frac{1}{\theta^{3}}\right)\left(1-e^{-2 \theta}\right)-\frac{2}{\theta^{2}}, \\
& \mathbb{E}^{\mathbb{Q}}\left(G_{2} G_{3}\right)=\int_{0}^{1}\left(\left(1+\frac{1}{\theta}\right) e^{\theta(s-1)}-\frac{1}{\theta}\right) \times\left(\left(1+\frac{2}{\theta}+\frac{2}{\theta^{2}}\right) e^{\theta(s-1)}-\left(\frac{2 s}{\theta}+\frac{2}{\theta^{2}}\right)\right) d s
\end{aligned}
$$




$$
\begin{aligned}
=\int_{0}^{1}\left(1+\frac{1}{\theta}\right)\left(1+\frac{2}{\theta}+\frac{2}{\theta^{2}}\right) e^{2 \theta(s-1)}- & \frac{1}{\theta}\left(1+\frac{2}{\theta}+\frac{2}{\theta^{2}}\right) e^{\theta(s-1)} \\
-\left(1+\frac{1}{\theta}\right) e^{\theta(s-1)}\left(\frac{2 s}{\theta}+\frac{2}{\theta^{2}}\right)+ & \frac{1}{\theta}\left(\frac{2 s}{\theta}+\frac{2}{\theta^{2}}\right) d s \\
=\left[\left(1+\frac{1}{\theta}\right)\left(1+\frac{2}{\theta}+\frac{2}{\theta^{2}}\right) \frac{e^{2 \theta(s-1)}}{2 \theta}-\right. & \left(\frac{1}{\theta}+\frac{2}{\theta^{2}}+\frac{2}{\theta^{3}}\right) \frac{e^{\theta(s-1)}}{\theta} \\
& \left.-\left(1+\frac{1}{\theta}\right) \frac{2 s}{\theta^{2}} e^{\theta(s-1)}+\left(\frac{s^{2}}{\theta^{2}}+\frac{2 s}{\theta^{3}}\right)\right]_{0}^{1}
\end{aligned}
$$

Proof of Lemma 2.4. The coefficient of $x^{2}$ in $P$ is

$$
\begin{aligned}
-A_{2}(\theta)=-h \frac{\theta}{6}+\frac{\sigma_{1}^{2}}{2} h \theta^{3}(- & \left.\frac{\alpha}{3}-\frac{\beta}{2}+a\right)^{2} \\
& +\frac{\sigma_{2}^{2}}{2} h \theta^{3}\left(b-\frac{\gamma}{2}-\frac{\theta^{2} \alpha \gamma}{2} \sigma_{1}^{2}\left(-\frac{\alpha}{3}-\frac{\beta}{2}+a\right)\right)^{2}+\frac{1}{2} h \theta^{3} c^{2} .
\end{aligned}
$$

We compute (using [WR15] software):

$$
\begin{gathered}
\alpha=\frac{1}{\sqrt{2 \theta}}+o\left(\frac{1}{\theta^{n}}\right), \forall n \geq 1 \\
\operatorname{Cov}^{\mathbb{Q}}\left(G_{1}, G_{3}\right)=\frac{1}{2 \theta}-\frac{1}{\theta^{2}}+\frac{1}{\theta^{3}}+o\left(\frac{1}{\theta^{3}}\right) \\
\beta=\frac{1}{\sqrt{2 \theta}}-\frac{\sqrt{2}}{\theta^{3 / 2}}+\frac{\sqrt{2}}{\theta^{5 / 2}}+o\left(\frac{1}{\theta^{5 / 2}}\right), \\
\beta^{2}=\frac{1}{2 \theta}-\frac{2}{\theta^{2}}+\frac{4}{\theta^{3}}-\frac{4}{\theta^{4}}+o\left(\frac{1}{\theta^{4}}\right) \\
\operatorname{Var}^{\mathbb{Q}}\left(G_{3}\right)=\frac{1}{2 \theta}-\frac{2}{3 \theta^{2}}+o\left(\frac{1}{\theta^{4}}\right) \\
\gamma=\frac{2}{\theta \sqrt{3}}-\frac{\sqrt{3}}{\theta^{2}}+\frac{\sqrt{3}}{4 \theta^{3}}+\frac{3 \sqrt{3}}{8 \theta^{4}}+o\left(\frac{1}{\theta^{4}}\right) \\
\operatorname{Cov}^{\mathbb{Q}}\left(G_{1}, G_{2}\right)=\frac{1}{2 \theta}-\frac{1}{2 \theta^{2}}+o\left(\frac{1}{\theta^{3}}\right) \\
\sigma_{1}^{2}=\frac{6}{\theta}+\frac{18}{\theta^{2}}+\frac{18}{\theta^{3}}-\frac{54}{\theta^{4}}+o\left(\frac{1}{\theta^{4}}\right) \\
a=\frac{1}{\sqrt{2 \theta}}-\frac{1}{\theta^{3 / 2} \sqrt{2}}+o\left(\frac{1}{\theta^{5 / 2}}\right) \\
\operatorname{Var}^{\mathbb{Q}}\left(G_{2}\right)=\frac{1}{2 \theta}-\frac{3}{2 \theta^{3}}+o\left(\frac{1}{\theta^{3}}\right) \\
b=\frac{\sqrt{3}}{2 \theta}-\frac{\sqrt{3}}{4 \theta^{2}}-\frac{9 \sqrt{3}}{16 \theta^{3}}+o\left(\frac{1}{\theta^{3}}\right) \\
c^{2}=\frac{1}{4 \theta^{2}}-\frac{5}{4 \theta^{3}}+o\left(\frac{1}{\theta^{3}}\right) \\
\sigma_{2}^{2}-\frac{1}{\theta}+2+o\left(\frac{1}{\theta^{3}}\right) \\
b
\end{gathered}
$$


From which we deduce

$$
\begin{gathered}
b-\frac{\gamma}{2}-\frac{\theta^{2} \alpha \gamma}{2} \sigma_{1}^{2}\left(-\frac{\alpha}{3}-\frac{\beta}{2}+a\right)=\frac{\sqrt{3}}{2 \theta^{3}}+o\left(\frac{1}{\theta^{3}}\right) \\
\frac{\sigma_{2}^{2}}{2} h \theta^{3}\left(b-\frac{\gamma}{2}-\frac{\theta^{2} \alpha \gamma}{2} \sigma_{1}^{2}\left(-\frac{\alpha}{3}-\frac{\beta}{2}+a\right)\right)^{2} \underset{\theta \rightarrow+\infty}{\longrightarrow} 0 \\
\frac{1}{2} h \theta^{3} c^{2}=h\left(\frac{\theta}{8}-\frac{5}{8}+o(1)\right) \\
\frac{\sigma_{1}^{2}}{2} h \theta^{3}\left(-\frac{\alpha}{3}-\frac{\beta}{2}+a\right)^{2}=h\left(\frac{\theta}{24}+\frac{1}{8}+o(1)\right) \\
A_{2}(\theta) \underset{\theta \rightarrow+\infty}{\longrightarrow} \frac{h}{2}
\end{gathered}
$$

The coefficient of $z^{2}$ in $P$ is

$$
-B_{2}(\theta)=-h \frac{\theta}{6}+\frac{\sigma_{1}^{2}}{2} h \theta^{3}\left(-\frac{\alpha}{6}+\frac{\beta}{2}\right)^{2}+\frac{\sigma_{2}^{2}}{2} h \theta^{3}\left(\frac{\gamma}{2}-\frac{\theta^{2} \alpha \gamma}{2} \sigma_{1}^{2}\left(-\frac{\alpha}{6}+\frac{\beta}{2}\right)\right)^{2} .
$$

We have:

$$
\begin{gathered}
\frac{\sigma_{2}^{2}}{2} h \theta^{3}\left(\frac{\gamma}{2}-\frac{\theta^{2} \alpha \gamma}{2} \sigma_{1}^{2}\left(-\frac{\alpha}{6}+\frac{\beta}{2}\right)\right)^{2} \underset{\theta \rightarrow+\infty}{\longrightarrow} 0, \\
\frac{\sigma_{1}^{2}}{2} h \theta^{3}\left(-\frac{\alpha}{6}+\frac{\beta}{2}\right)^{2}=h\left(\frac{\theta}{6}-\frac{1}{2}+o(1)\right), \\
B_{2}(\theta) \underset{\theta \rightarrow+\infty}{\longrightarrow} \frac{h}{2} .
\end{gathered}
$$

The coefficient of $x z$ in $P$ is

$$
\begin{aligned}
C_{1}(\theta)=-h \frac{\theta}{6} & +\sigma_{1}^{2} h \theta^{3}\left(-\frac{\alpha}{3}-\frac{\beta}{2}+a\right)\left(-\frac{\alpha}{6}+\frac{\beta}{2}\right) \\
& +\sigma_{2}^{2} h \theta^{3}\left(b-\frac{\gamma}{2}-\frac{\theta^{2} \alpha \gamma}{2} \sigma_{1}^{2}\left(-\frac{\alpha}{3}-\frac{\beta}{2}+a\right)\right)\left(\frac{\gamma}{2}-\frac{\theta^{2} \alpha \gamma}{2} \sigma_{1}^{2}\left(-\frac{\alpha}{6}+\frac{\beta}{2}\right)\right)
\end{aligned}
$$

(it does not depend on $y_{0: \tau}$ ). We have:

$$
\begin{gathered}
\sigma_{2}^{2} h \theta^{3}\left(b-\frac{\gamma}{2}-\frac{\theta^{2} \alpha \gamma}{2} \sigma_{1}^{2}\left(-\frac{\alpha}{3}-\frac{\beta}{2}+a\right)\right)\left(\frac{\gamma}{2}-\frac{\theta^{2} \alpha \gamma}{2} \sigma_{1}^{2}\left(-\frac{\alpha}{6}+\frac{\beta}{2}\right)\right) \underset{\theta \rightarrow+\infty}{\rightarrow} 0 \\
\sigma_{1}^{2} h \theta^{3}\left(-\frac{\alpha}{3}-\frac{\beta}{2}+a\right)\left(-\frac{\alpha}{6}+\frac{\beta}{2}\right)=h\left(\frac{\theta}{6}-\frac{3}{2 \theta}+o\left(\frac{1}{\theta}\right)\right) \\
C_{1}(\theta)=\frac{3 h}{2 \theta}+o\left(\frac{1}{\theta}\right) .
\end{gathered}
$$

We need the following Lemma before going into the proof of Lemma 2.7.

Lemma 7.3. For all $k \in \mathbb{N}, s \in[k, k+1]$

$$
\left|Y_{\tau s}-\int_{k}^{k+1} Y_{\tau u} d u-Y_{\tau(s+1)}+\int_{k}^{k+1} Y_{\tau(u+1)} d u\right| \preceq h \tau^{2} M+h \tau \mathcal{V}_{k \tau,(k+1) \tau}+\mathcal{W}_{k \tau,(k+1) \tau},
$$




$$
\left|\int_{0}^{1} \frac{e^{-\theta} \sinh (\theta s)}{\theta} d Y_{\tau k+\tau s}-\int_{0}^{1} \frac{e^{-\theta} \sinh (\theta s)}{\theta} d Y_{\tau(k+1)+\tau s}\right| \preceq \frac{M \tau+\mathcal{V}_{k \tau,(k+1) \tau}+\mathcal{W}_{k \tau,(k+1) \tau}}{\theta} .
$$

And, for all $s \in[0,1]$,

$$
\begin{aligned}
& \left|Y_{\tau s}-\int_{0}^{1} Y_{\tau u} d u\right| \preceq h \tau^{2} M+h \tau \mathcal{V}_{0, \tau}+\mathcal{W}_{0, \tau}, \\
& \left|\int_{0}^{1} \frac{e^{-\theta} \sinh (\theta s)}{\theta} d Y_{\tau s}\right| \preceq \frac{M \tau+\mathcal{V}_{0, \tau}+\mathcal{W}_{0, \tau}}{\theta} .
\end{aligned}
$$

Proof. We write the proof only for the first two formulas. We have, for all $k \in \mathbb{N}, s \in[k, k+1]$,

$$
\begin{gathered}
\left|Y_{\tau s}-\int_{k}^{k+1} Y_{\tau u} d u-Y_{\tau(s+1)}+\int_{k}^{k+1} Y_{\tau(u+1)} d u\right| \\
=\left|-\int_{\tau s}^{\tau(s+1)} h X_{u} d u+W_{\tau s}-W_{\tau(s+1)}-\int_{k}^{k+1}\left(-h \int_{\tau u}^{\tau(u+1)} X_{v} d v+W_{\tau u}-W_{\tau(u+1)}\right) d u\right| \\
\preceq\left|\int_{k}^{k+1} h\left(\int_{\tau s}^{\tau(s+1)} X_{v} d v-\int_{\tau u}^{\tau(u+1)} X_{v} d v\right) d u\right|+\mathcal{W}_{k \tau,(k+2) \tau} \\
=\left|h \int_{k}^{k+1}\left(\int_{\tau s}^{\tau(s+1)} X_{v}-X_{v+\tau(u-s)} d v\right) d u\right|+\mathcal{W}_{k \tau,(k+2) \tau} \\
=\left|h \int_{k}^{k+1}\left(\int_{\tau s}^{\tau(s+1)} \int_{v+\tau(u-s)}^{v} f\left(X_{t}\right) d t+V_{v}-V_{v+\tau(u-s)} d v d u\right)\right|+\mathcal{W}_{k \tau,(k+2) \tau} \\
\leq h \tau^{2} M+h \tau \mathcal{V}_{k \tau,(k+2) \tau}+\mathcal{W}_{k \tau,(k+2) \tau},
\end{gathered}
$$

and (using integration by parts)

$$
\begin{gathered}
\left|\int_{0}^{1} \frac{e^{-\theta} \sinh (\theta s)}{\theta} d Y_{\tau k+\tau s}-\int_{0}^{1} \frac{e^{-\theta} \sinh (\theta s)}{\theta} d Y_{\tau(k+1)+\tau s}\right| \\
=\left|\int_{0}^{\tau} \frac{e^{-\theta} \sinh (h s)}{\theta}\left(h\left(X_{\tau k+s}-X_{\tau(k+1)+s}\right) d s+d W_{\tau k+s}-d W_{\tau(k+1)+s}\right)\right| \\
\leq\left|\int_{0}^{\tau} \frac{e^{-\theta} \sinh (h s)}{\theta} h\left(X_{\tau k+s}-X_{\tau(k+1)+s}\right) d s\right| \\
\quad+\left|\frac{e^{-\theta} \sinh (\theta)}{\theta}\left(W_{\tau(k+1)}-W_{\tau(k+2)}\right)\right| \\
+\left|\int_{0}^{\tau}\left(W_{\tau k+s}-W_{\tau(k+1)+s}\right) \frac{e^{-\theta} \cosh (h s)}{\tau} d s\right| \\
\preceq \int_{0}^{\tau} \frac{e^{-\theta} \sinh (h s)}{\theta} h\left(\tau M+\mathcal{V}_{k \tau,(k+2) \tau}\right) d s+\frac{e^{-\theta} \sinh (\theta)}{\theta} \mathcal{W}_{k \tau,(k+2) \tau}+\int_{0}^{\tau} \mathcal{W}_{k \tau,(k+2) \tau} \frac{e^{-\theta} \cosh (h s)}{\tau} d s \\
\preceq \frac{h\left(M \tau+\mathcal{V}_{k \tau,(k+2) \tau}\right)}{h \theta}+\frac{\mathcal{W}_{k \tau,(k+2) \tau}}{\theta} .
\end{gathered}
$$

Proof of Lemma 2.7. We write the proof in the case $k=0$. From (2.3), (2.15), we deduce

$$
\begin{aligned}
B_{1}\left(Y_{0: \tau}, \theta\right)=- & \sigma_{1}^{2} h \theta^{2}\left(-\frac{\alpha}{6}+\frac{\beta}{2}\right) \lambda_{1}\left(Y_{0: \tau}\right)+h \int_{0}^{1} s d Y_{\tau s} \\
& +\sigma_{2}^{2} h \theta^{2}\left(\frac{\gamma}{2}-\frac{\theta^{2} \alpha \gamma \sigma_{1}^{2}}{2}\left(-\frac{\alpha}{6}+\frac{\beta}{2}\right)\right)\left(-\lambda_{2}\left(Y_{0: \tau}\right)+\frac{\theta^{2} \alpha \gamma \sigma_{1}^{2}}{2} \lambda_{1}\left(Y_{0: \tau}\right)\right) .
\end{aligned}
$$


For further use, we also write the formula for $A_{1}\left(Y_{0: \tau}, \theta\right)$ :

$$
\begin{aligned}
& A_{1}\left(Y_{0: \tau}, \theta\right)=h \int_{0}^{1}(1-s) d Y_{s \tau}+\sigma_{1}^{2} h \theta^{2}\left(\frac{\alpha}{3}+\frac{\beta}{2}-a\right) \lambda_{1}\left(Y_{0: \tau}\right) \\
& +\sigma_{2}^{2} h \theta^{2}\left(b-\frac{\gamma}{2}-\frac{\theta^{2} \alpha \gamma \sigma_{1}^{2}}{2}\left(-\frac{\alpha}{3}-\frac{\beta}{2}+a\right)\right)\left(-\lambda_{2}\left(Y_{0: \tau}\right)+\frac{\theta^{2} \alpha \gamma \sigma_{1}^{2}}{2} \lambda_{1}\left(Y_{0: \tau}\right)\right)-h \theta^{2} \lambda_{3}\left(Y_{0: \tau}\right) c .
\end{aligned}
$$

We have to remember here that $\lambda_{1}, \lambda_{2}, \lambda_{3}$ are functions of $y_{0: \tau}$. So we might write $\lambda_{1}\left(y_{0: \tau}\right), \ldots$ to stress this dependency (and the same goes for other quantities). From Lemmas 7.1, 7.2, we get $\left(g_{1}, g_{2}\right.$ defined below)

$$
\begin{aligned}
& \operatorname{Cov}^{\mathbb{Q}}\left(G_{1}, G_{4}\right)\left(Y_{0: \tau}\right)=\int_{0}^{1} e^{\theta(s-1)} \times\left(g_{1}(s)-\theta e^{\theta s} \int_{s}^{1} e^{-\theta u} g_{1}(u) d u\right) d s \\
&=\int_{0}^{1} g_{1}(s) e^{\theta(s-1)} d s-\int_{0}^{1} e^{-\theta u} g_{1}(u) \int_{0}^{u} \theta e^{2 \theta s-\theta} d s d u \\
&=\int_{0}^{1} g_{1}(s) e^{\theta(s-1)} d s-\int_{0}^{1} e^{-\theta u} g_{1}(u)\left(\frac{e^{2 \theta u}-1}{2}\right) e^{-\theta} d u \\
&=\int_{0}^{1} g_{1}(s) e^{-\theta} \cosh (\theta s) d s \\
& \operatorname{Cov}^{\mathbb{Q}}\left(G_{1}, G_{4}\right)\left(Y_{\tau: 2 \tau}\right)=\int_{0}^{1} g_{2}(s) e^{-\theta} \cosh (\theta s) d s
\end{aligned}
$$

with

$$
g_{1}(s)=Y_{\tau s}-\int_{0}^{1} Y_{\tau u} d u, g_{2}(s)=Y_{\tau(s+1)}-\int_{0}^{1} Y_{\tau(u+1)} d u,
$$

and

$$
\begin{aligned}
\operatorname{Cov}\left(G_{3}, G_{4}\right)\left(Y_{0: \tau}\right)= & \int_{0}^{1}\left(\left(1+\frac{2}{\theta}+\frac{2}{\theta^{2}}\right) e^{\theta(s-1)}-\left(\frac{2 s}{\theta}+\frac{2}{\theta^{2}}\right)\right) \\
& \times\left(g_{1}(s)-\theta e^{\theta s} \int_{s}^{1} e^{-\theta u} g_{1}(u) d u\right) d s \\
= & \left(1+\frac{2}{\theta}+\frac{2}{\theta^{2}}\right) \operatorname{Cov}\left(G_{1}, G_{4}\right)\left(Y_{0: \tau}\right)-\int_{0}^{1} \frac{2 s}{\theta} g_{1}(s) d s \\
& \quad+\int_{0}^{1} e^{-\theta u} g_{1}(u) \int_{0}^{u}\left(2 s+\frac{2}{\theta}\right) e^{\theta s} d s d u \\
= & \left(1+\frac{2}{\theta}+\frac{2}{\theta^{2}}\right) \operatorname{Cov}\left(G_{1}, G_{4}\right)\left(Y_{0: \tau}\right)-\int_{0}^{1} \frac{2 s}{\theta} g_{1}(s) d s \\
& +\int_{0}^{1} e^{-\theta u} g_{1}(u) \frac{2 u}{\theta} e^{\theta u} d u \\
= & =\left(1+\frac{2}{\theta}+\frac{2}{\theta^{2}}\right) \operatorname{Cov}\left(G_{1}, G_{4}\right)\left(Y_{0: \tau}\right),
\end{aligned}
$$

$$
\operatorname{Cov}\left(G_{3}, G_{4}\right)\left(Y_{\tau: 2 \tau}\right)=\left(1+\frac{2}{\theta}+\frac{2}{\theta^{2}}\right) \operatorname{Cov}\left(G_{1}, G_{4}\right)\left(Y_{\tau: 2 \tau}\right) .
$$

From (2.8), (7.5)-(7.17), we deduce (using again [WR15])

$$
-\sigma_{1}^{2} h \theta^{2}\left(-\frac{\alpha}{6}+\frac{\beta}{2}\right) \lambda_{1}\left(Y_{0: \tau}\right)=-h(2 \theta+O(1)) \operatorname{Cov}^{\mathbb{Q}}\left(G_{1}, G_{4}\right)\left(Y_{0: \tau}\right),
$$




$$
\begin{aligned}
\sigma_{2}^{2} h \theta^{2} & \left(\frac{\gamma}{2}-\frac{\theta^{2} \alpha \gamma \sigma_{1}^{2}}{2}\left(-\frac{\alpha}{6}+\frac{\beta}{2}\right)\right)=h O(\theta), \\
-\lambda_{2}\left(Y_{0: \tau}\right)+\frac{\theta^{2} \alpha \gamma \sigma_{1}^{2}}{2} \lambda_{1}\left(Y_{0: \tau}\right) & =\operatorname{Cov}^{\mathbb{Q}}\left(G_{1}, G_{4}\right)\left(Y_{0: \tau}\right)\left(-\frac{1}{\gamma}\left(1+\frac{2}{\theta}+\frac{2}{\theta^{2}}-\frac{\beta}{\alpha}\right)+\frac{\beta}{\alpha \gamma}+\frac{\theta^{2} \gamma \sigma_{1}^{2}}{2}\right) \\
& =\operatorname{Cov}^{\mathbb{Q}}\left(G_{1}, G_{4}\right)\left(Y_{0: \tau}\right) \times O\left(\frac{1}{\theta}\right) .
\end{aligned}
$$

So we get

$$
\begin{aligned}
-\sigma_{1}^{2} h \theta^{2}\left(-\frac{\alpha}{6}+\frac{\beta}{2}\right) \lambda_{1}\left(Y_{0: \tau}\right)+\sigma_{2}^{2} h \theta^{2}\left(\frac{\gamma}{2}-\frac{\theta^{2} \alpha \gamma \sigma_{1}^{2}}{2}\right. & \left.\left(-\frac{\alpha}{6}+\frac{\beta}{2}\right)\right)\left(-\lambda_{2}\left(Y_{0: \tau}\right)+\frac{\theta^{2} \alpha \gamma \sigma_{1}^{2}}{2} \lambda_{1}\left(Y_{0: \tau}\right)\right) \\
& =-2 h(\theta+O(1)) \operatorname{Cov}^{\mathbb{Q}}\left(G_{1}, G_{4}\right)\left(Y_{0: \tau}\right) .
\end{aligned}
$$

We have

$$
\begin{aligned}
\operatorname{Cov}^{\mathbb{Q}}\left(G_{1}, G_{4}\right)\left(Y_{0: \tau}\right) & =\int_{0}^{1}\left(Y_{\tau s}-\int_{0}^{1} Y_{\tau u} d u\right) e^{-\theta} \cosh (\theta s) d s \\
& =\left(Y_{\tau}-\int_{0}^{1} Y_{\tau u} d u\right) \frac{e^{-\theta} \sinh (\theta)}{\theta}-\int_{0}^{1} \frac{e^{-\theta} \sinh (\theta s)}{\theta} d Y_{\tau s} \\
& =\int_{0}^{1} s d Y_{\tau s} \times \frac{e^{-\theta} \sinh (\theta)}{\theta}-\int_{0}^{1} \frac{e^{-\theta} \sinh (\theta s)}{\theta} d Y_{\tau s},
\end{aligned}
$$

and so

$$
\begin{aligned}
-2 h(\theta+O(1)) \operatorname{Cov}^{\mathbb{Q}}\left(G_{1}, G_{4}\right)\left(Y_{0: \tau}\right)+h \int_{0}^{1} s d Y_{\tau s}=-2 h(\theta+O(1))\left(\int_{0}^{1} s d Y_{\tau s}\right)\left(\frac{1}{2 \theta}-\frac{e^{-2 \theta}}{2 \theta}\right) \\
+h \int_{0}^{1} s d Y_{\tau s}+h(\theta+O(1)) \times 2 \int_{0}^{1} \frac{e^{-\theta} \sinh (\theta s)}{\theta} d Y_{\tau s} \\
=h\left(\int_{0}^{1} s d Y_{\tau s}\right) \times O\left(\frac{1}{\theta}\right)-h(\theta+O(1)) \times 2 \int_{0}^{1} \frac{e^{-\theta} \sinh (\theta s)}{\theta} d Y_{\tau s}
\end{aligned}
$$

And so, using Lemma 7.3, Equations (7.30), (7.31), (7.32) (as similar formulas of the ones above are valid if we replace $Y_{0: \tau}$ by $\left.Y_{0: 2 \tau}\right)$, we get

$$
\begin{aligned}
\left|B_{1}\left(Y_{0: \tau}, \theta\right)-B_{1}\left(Y_{\tau: 2 \tau}, \theta\right)\right| & \preceq \frac{1}{\tau}\left(h \tau^{2} M+h \tau \mathcal{V}_{0,2 \tau}+\mathcal{W}_{0,2 \tau}\right)+h \theta\left(\frac{M \tau+\mathcal{V}_{0,2 \tau}+\mathcal{W}_{0,2 \tau}}{\theta}\right) \\
& \preceq M h \tau+h \mathcal{V}_{0,2 \tau}+\left(h+\frac{1}{\tau}\right) \mathcal{W}_{0,2 \tau}
\end{aligned}
$$

\subsection{Technical Lemmas used in Section 4}

Lemma 7.4. We have $\lambda_{1}=\int_{0}^{\tau} f_{1}(s) d y_{\tau s}$ for some deterministic function $f_{1}$. And the same is true for $\lambda_{2}, \lambda_{3}$.

Proof. We write the proof only for $\lambda_{1}$. Using Equation (2.8) and Lemmas 7.1, 7.2 and integrations by parts, we get

$$
\begin{aligned}
\alpha \lambda_{1} & =\operatorname{Cov}^{\mathbb{Q}}\left(G_{1}, G_{4}\right) \\
& =\operatorname{Cov}^{\mathbb{Q}}\left(B_{1}, \int_{0}^{1} y_{\tau s}-\left(\int_{0}^{1} y_{\tau u} d u\right) d B_{s}\right)
\end{aligned}
$$




$$
\begin{aligned}
= & \operatorname{Cov}^{\mathbb{Q}}\left(\int_{0}^{1} e^{\theta(s-1)} d \beta_{s}, \int_{0}^{1} y_{\tau s}-\left(\int_{0}^{1} y_{\tau u} d u\right)-\theta e^{\theta s} \int_{s}^{1} e^{-\theta u}\left(y_{\tau u}-\left(\int_{0}^{1} y_{\tau v} d v\right)\right) d u d \beta_{s}\right) \\
= & \int_{0}^{1} e^{\theta(s-1)}\left(y_{\tau s}-\left(\int_{0}^{1} y_{\tau u} d u\right)-\theta e^{\theta s} \int_{s}^{1} e^{-\theta u}\left(y_{\tau u}-\left(\int_{0}^{1} y_{\tau v} d v\right)\right) d u\right) d s \\
= & \int_{0}^{1} e^{\theta(s-1)} y_{\tau s} d s-\int_{0}^{1} e^{\theta(s-1)} d s \times \int_{0}^{1} y_{\tau u} d u-\int_{0}^{1} e^{-\theta u} y_{\tau u} \int_{u}^{1} \theta e^{\theta(2 s-1)} d s d u \\
& \quad-\int_{0}^{1} \theta e^{\theta(2 s-1)} \frac{\left(e^{-\theta s}-e^{-\theta}\right)}{\theta} d s \times \int_{0}^{1} y_{\tau u} d u \\
= & \frac{y_{\tau}}{\theta}-\int_{0}^{1} \frac{e^{\theta(s-1)}}{\theta} d y_{\tau s}-\frac{\left(1-e^{-\theta}\right)}{\theta}\left(y_{\tau}-\int_{0}^{1} u d y_{\tau u}\right)-\int_{0}^{1} y_{\tau u} \sinh (\theta(1-u)) d u \\
& \quad-\left(\frac{1-e^{-\theta}}{\theta}-\frac{\left(1-e^{-2 \theta}\right)}{2 \theta}\right)\left(y_{\tau}-\int_{0}^{1} u d y_{\tau u}\right) \\
= & \frac{y_{\tau}}{\theta}-\int_{0}^{1} \frac{e^{\theta(s-1)}}{\theta} d y_{\tau s}-\frac{\left(1-e^{-\theta}\right)}{\theta}\left(y_{\tau}-\int_{0}^{1} u d y_{\tau u}\right)+\frac{y_{\tau}}{\theta}-\int_{0}^{1} \frac{\cosh (\theta(1-u))}{\theta} d y_{\tau u} \\
& \quad-\left(\frac{1-e^{-\theta}}{\theta}-\frac{\left(1-e^{-2 \theta}\right)}{2 \theta}\right)\left(y_{\tau}-\int_{0}^{1} u d y_{\tau u}\right) .
\end{aligned}
$$

Lemma 7.5. We can write

$$
A_{1}^{Y_{0: \tau}}=\int_{0}^{\tau}\left(f_{1}(s) d W_{s}+f_{2}(s)\right) d X_{s}
$$

for some deterministic functions $f_{1}, f_{2}$.

Proof. From Lemma 7.4 and Equation (7.24), we know there exists a deterministic function $g_{1}$ such that

$$
A_{1}^{Y_{0: \tau}}=\int_{0}^{1} g_{1}(s) d Y_{\tau s} .
$$

So, integrating by parts, we get (where $G$ is the antiderivative of $g_{1}$ such that $G(0)=0$ )

$$
\begin{aligned}
A_{1}^{Y_{0: \tau}} & =\int_{0}^{1} g_{1}(s)\left(h X_{\tau s} \tau d s+d W_{\tau s}\right) \\
& =G(1) h X_{\tau} \tau-\int_{0}^{1} G(s) h \tau d X_{\tau s}+\int_{0}^{1} g_{1}(s) d W_{\tau s} \\
& \left.=\int_{0}^{\tau}\left(G(1) h-G\left(\frac{s}{\tau}\right) h\right)\right) d X_{s}+\int_{0}^{\tau} g_{1}\left(\frac{s}{\tau}\right) d W_{s} .
\end{aligned}
$$

Lemma 7.6. For any measurable $\mathcal{B}$, subset of $\mathbb{R}^{2}$ and $\Phi$ a Gaussian density,

$$
\begin{aligned}
\int_{\left(t_{1}, t_{2}\right) \in \mathcal{B}} \Phi\left(t_{1}, t_{2}\right) & \Psi_{1}\left(t_{1}, t_{2}\right) d t_{1} d t_{2} \\
& \leq \int_{\mathcal{B}} \Phi\left(t_{1}, t_{2}\right) C_{1}^{\prime}(h, \tau) \exp \left(2 M\left|(1,-1) \kappa^{-1}\left(t_{1}, t_{2}\right)^{T}\right|+\tau\left(2 M+M^{2}\right)\right) d t_{1} d t_{2}
\end{aligned}
$$

(the constant $C_{1}^{\prime}(h, \tau)$ coming from Lemma 4.3).

Proof. For any $\epsilon^{\prime}<\epsilon\left(\epsilon\right.$ comes from Lemma 4.3), we can write $\mathcal{B}=\sqcup_{i \in I} \mathcal{B}_{i}$, where $I \subset \mathbb{N}, \sqcup$ means that this is a partition, and for all $i$, the set $\mathcal{B}_{i}$ is a subset of $B\left(2\left(x_{i}, z_{i}\right) \kappa, \epsilon^{\prime}\right)$ (for some $\left.x_{i}, z_{i}\right)$. For all $i$, for almost all $\left(t_{1}, t_{2}\right)$ in $B\left(2\left(x_{i}, z_{i}\right) \kappa, \epsilon^{\prime}\right)$, we have, by Lemma 4.3 ,

$$
\Psi_{1}\left(t_{1}, t_{2}\right) \leq C_{1}^{\prime}(h, \tau) \exp \left(4 M\left|x_{i}-z_{i}\right|+\tau\left(2 M+M^{2}\right)\right)
$$




$$
\begin{aligned}
& =C_{1}^{\prime}(h, \tau) \exp \left(4 M\left|\left(x_{i}, z_{i}\right)(1,-1)^{T}\right|+\tau\left(2 M+M^{2}\right)\right) \\
& =C_{1}^{\prime}(h, \tau) \exp \left(4 M\left|2\left(x_{i}, z_{i}\right) \kappa \frac{1}{2} \kappa^{-1}(1,-1)^{T}\right|+\tau\left(2 M+M^{2}\right)\right) \\
& \leq C_{1}^{\prime}(h, \tau) \exp \left(4 M\left|\left(t_{1}, t_{2}\right) \frac{1}{2} \kappa^{-1}(1,-1)^{T}\right|+C^{\prime} \epsilon^{\prime}+\tau\left(2 M+M^{2}\right)\right),
\end{aligned}
$$

for some constant $C^{\prime}$. So we have

$$
\begin{aligned}
& \int_{\left(t_{1}, t_{2}\right) \in \mathcal{B}} \Phi\left(t_{1}, t_{2}\right) \Psi_{1}\left(t_{1}, t_{2}\right) d t_{1} d t_{2}=\sum_{i \in I} \int_{\left(t_{1}, t_{2}\right) \in \mathcal{B}_{i}} \Phi\left(t_{1}, t_{2}\right) \Psi_{1}\left(t_{1}, t_{2}\right) d t_{1} d t_{2} \\
& \leq \sum_{i \in I} \int_{\left(t_{1}, t_{2}\right) \in \mathcal{B}_{i}} \Phi\left(t_{1}, t_{2}\right) C_{1}^{\prime}(h, \tau) \exp \left(4 M\left|\left(t_{1}, t_{2}\right) \frac{1}{2} \kappa^{-1}(1,-1)^{T}\right|+C^{\prime} \epsilon^{\prime}+\tau\left(2 M+M^{2}\right)\right) d t_{1} d t_{2} \\
& =\int_{\left(t_{1}, t_{2}\right) \in \mathcal{B}} \Phi\left(t_{1}, t_{2}\right) C_{1}^{\prime}(h, \tau) \exp \left(4 M\left|\left(t_{1}, t_{2}\right) \frac{1}{2} \kappa^{-1}(1,-1)^{T}\right|+C^{\prime} \epsilon^{\prime}+\tau\left(2 M+M^{2}\right)\right) d t_{1} d t_{2} .
\end{aligned}
$$

And by taking $\epsilon^{\prime} \rightarrow 0$, we get the desired result.

\subsection{Technical Lemma used in Section 6}

Lemma 7.7. Suppose that $f_{1}$ and $f_{2}$ are two probability densities on $\mathbb{R}_{+}$and that $\varphi$ is a nondecreasing function from $\mathbb{R}_{+}$to $\mathbb{R}_{+}$. Suppose the distribution functions $F_{1}$ an $F_{2}$ (associated respectively to $f_{1}, f_{2}$ ) are such that

$$
1-F_{1}(x) \leq K\left(1-F_{2}(x)\right), \text { for all } x \geq 0,
$$

for some constant $K \geq 1$. Then, for $X_{1}$ a random variable of density $f_{1}$ and $X_{2}$ a random variable of density $f_{2}$, we have

$$
\mathbb{E}\left(\varphi\left(X_{1}\right)\right) \leq K \mathbb{E}\left(\varphi\left(X_{2}\right)\right) .
$$

Proof. Let $U$ be a random variable of uniform law on $[0,1]$. Let $m$ be such that $F_{2}(m)=1-1 / K$. Let $\left(K\left(1-F_{2}\right)\right)^{-1}$ be the inverse of $\widehat{F}_{2}: x \in\left[m,+\infty\left[\mapsto K\left(1-F_{2}(x)\right) \in[0,1]\right.\right.$. We have:

$$
\begin{aligned}
\mathbb{E}\left(\varphi\left(X_{1}\right)\right) & =\mathbb{E}\left(\varphi\left(\left(1-F_{1}\right)^{-1}(U)\right)\right. \\
& \leq \mathbb{E}\left(\varphi\left(\left(K\left(1-F_{2}\right)\right)^{-1}(U)\right)\right) \\
& =\mathbb{E}\left(\varphi\left(X_{2}\right) \mid X_{2} \geq m\right) \\
& =\mathbb{E}\left(\varphi\left(X_{2}\right) \mathbb{1}_{[m,+\infty[}\left(X_{2}\right)\right) \times K \\
& \leq K \mathbb{E}\left(\varphi\left(X_{2}\right)\right) .
\end{aligned}
$$

THANKS : The authors would like to thank the following colleagues, whose help was greatly appreciated: Dan Crisan, François Delarue.

\section{References}

[Ata98] Rami Atar. Exponential stability for nonlinear filtering of diffusion processes in a noncompact domain. Ann. Probab., 26(4):1552-1574, 1998.

[AZ97] Rami Atar and Ofer Zeitouni. Exponential stability for nonlinear filtering. Ann. Inst. H. Poincaré Probab. Statist., 33(6):697-725, 1997.

[BC09] Alan Bain and Dan Crisan. Fundamentals of stochastic filtering, volume 60 of Stochastic Modelling and Applied Probability. Springer, New York, 2009. 
[BCL04] Peter Baxendale, Pavel Chigansky, and Robert Liptser. Asymptotic stability of the Wonham filter: ergodic and nonergodic signals. SIAM J. Control Optim., 43(2):643$669,2004$.

[Ben81] V. E. Beneš. Exact finite-dimensional filters for certain diffusions with nonlinear drift. Stochastics, 5(1-2):65-92, 1981.

[CR11] D. Crisan and B. Rozovski1. Introduction. In The Oxford handbook of nonlinear filtering, pages 1-15. Oxford Univ. Press, Oxford, 2011.

[CvH07] Pavel Chigansky and Ramon van Handel. Model robustness of finite state nonlinear filtering over the infinite time horizon. Ann. Appl. Probab., 17(2):688-715, 2007.

[DG01] Pierre Del Moral and Alice Guionnet. On the stability of interacting processes with applications to filtering and genetic algorithms. Ann. Inst. H. Poincaré Probab. Statist., 37(2):155-194, 2001.

[KV08] M. L. Kleptsyna and A. Yu. Veretennikov. On discrete time ergodic filters with wrong initial data. Probability Theory and Related Fields, 141(3):411-444, 2008.

[LO03] François LeGland and Nadia Oudjane. A robustification approach to stability and to uniform particle approximation of nonlinear filters: the example of pseudo-mixing signals. Stochastic Process. Appl., 106(2):279-316, 2003.

[MY08] Roger Mansuy and Marc Yor. Aspects of Brownian motion. Universitext. SpringerVerlag, Berlin, 2008.

[Oco99] D. L. Ocone. Asymptotic stability of Beneš filters. Stochastic Anal. Appl., 17(6):10531074, 1999.

[OR05] Nadia Oudjane and Sylvain Rubenthaler. Stability and uniform particle approximation of nonlinear filters in case of non ergodic signals. Stoch. Anal. Appl., 23(3):421-448, 2005.

[Oud00] Nadia Oudjane. Stabilité et approximations particulaires en filtrage non linéaire application au pistage. PhD thesis, 2000. Thèse de doctorat Mathématiques et application Rennes 1.

[Sta05] Wilhelm Stannat. Stability of the filter equation for a time-dependent signal on $\mathbb{R}^{d}$. Appl. Math. Optim., 52(1):39-71, 2005.

[WR15] Inc. Wolfram Research. Mathematica, 2015. 